\title{
Natural and synthetic nanoparticles for delivery of biologics
}

Linglei Jiang 


\section{Natural and synthetic nanoparticles for delivery of biologics Linglei Jiang}

PhD Thesis with summary in Dutch and English.

CDL Research, Division LAB, UMC Utrecht, Utrecht, the Netherlands Faculty of Medicine, Utrecht University, Utrecht, the Netherlands.

Dit proefschrift werd (mede) mogelijk gemaakt met financiële steun van China Scholarship Council.

The printing of this thesis was financially supported by UMC Utrecht.

ISBN: 978-94-6332-724-4

Cover design \& Thesis Lay-out: Iliana Boshoven-Gkini | AgileColor.com Printed by: GVO drukkers \& vormgevers B.V. | gvo.nl

Copyright (2020 Linglei Jiang

All rights reserved. No part of this publication may be reproduced or transmitted in any form by any means without prior permission of the author. 


\title{
Natural and synthetic nanoparticles for delivery of biologics
}

\section{Natuurlijke en synthetische nanodeeltjes voor de afgifte van biologische geneesmiddelen}

(met een samenvatting in het Nederlands)

\section{Proefschrift}

\author{
ter verkrijging van de graad van doctor aan de \\ Universiteit Utrecht \\ op gezag van de \\ rector magnificus, prof.dr. H.R.B.M. Kummeling, \\ ingevolge het besluit van het college voor promoties \\ in het openbaar te verdedigen op
}

dinsdag 12 januari 2021 des middags te 4.15 uur

door

\section{Linglei Jiang}

geboren op 7 november 1988

te Jianyang, China 


\section{Promotoren:}

Prof. dr. R.M. Schiffelers

Prof. dr. W.E. Hennink

\section{Copromotor:}

Dr. P. Vader 


\section{Table of Contents}

$\begin{array}{lll}\text { Chapter } 1 & \text { Introduction: nanomedicines and their clinical }\end{array}$ applications

Chapter 2 Poly(amido amine)-based nanogels for delivery of 17 mRNA

Chapter 3 Extracellular vesicles for nucleic acid delivery: progress and prospects for safe RNA-based gene therapy

Chapter 4 Bacterial membrane vesicles as promising vaccine candidates

Chapter 5 Membrane Vesicles as nano-weapon for combating

Chapter 6 A post-insertion strategy for surface functionalization of bacterial and mammalian cell-derived extracellular vesicles

Chapter 7 Immunomodulatory effects of Salmonella typhimurium OMVs in an intestine-mimicking co-culture system- a preliminary study

Chapter 8 Summary

$\begin{array}{lll}\text { Appendices } & \text { Samenvatting } & 146\end{array}$

$\begin{array}{ll}\text { Curriculum vitae } & 150\end{array}$

List of publications $\quad 151$

$\begin{array}{ll}\text { Acknowledgements } & 153\end{array}$ 
The original dream is tightly grasped in hand

The destination most desired How can we turn back at halfway The original dream will certainly be attained After fulfilling true desires Then it is considered that we attained heaven --- Lyrics from 'Original Dream' by Christine Fan, album released in 2004 


\section{Chapter 1}

Introduction: nanomedicines and their clinical applications 


\section{Chapter 1}

\section{Nanomedicines and their attractive advantages}

Nano is a prefix derives from the Greek word 'vâvos', which means 'drawf'. (Jain and Jain 2008) Nanomedicine is the healthcare application of nanoscale or nanostructure materials.(Wagner, Dullaart et al. 2006) It is an inter-disciplinary field involving chemistry, biology, physics, materials sciences and clinical medicine.(Farokhzad and Langer 2006) Nanomedicines can be used for both the diagnosis and therapy of diseases.

There are several attractive features of nanomedicines as drug delivery systems when compared with traditional small molecular weight drugs. For example, 1) they can increase the solubility of hydrophobic drugs; 2) effectively deliver drugs intracellularly and across barriers such as the blood-brain barrier; 3) be 'smart' by active targeting the site of disease, thus increase therapeutic outcome and simultaneously reduce side effects of drugs caused by accumulation in healthy tissues; 4) prolong circulation time by surface decoration drug-loaded nanoparticles with polyethylene glycol (PEG) that prevent aggregation, opsonization, and phagocytosis; (Suk, Xu et al. 2016); 5) overcome drug resistance as drug-loaded nanoparticles are uptake in a stealth endocytosis process that drugs become 'invisible' to drug efflux pumps.(Yang, Yi et al. 2014)

\section{Nanomedicine systems}

Nanomedicine can be mainly divided into two categories, i.e. synthetic nanomedicine system and natural nanomedicine system. Synthetic systems are mainly obtained by chemical synthesis methods, which can be further divided into 1) polymer-based nanocarriers such as micelles, nanogels and dendrimers; and 2) liposomes and lipidbased nanocarriers. Natural nanomedicine systems include, viruses, lipoproteins and extracellular vesicles (EVs). EVs can be released by different domains of life including eukaryotes, bacteria and fungi. (Deatherage and Cookson 2012, Brown, Wolf et al. 2015)

\section{Polymer-based systems}

The versatility of polymers makes them suitable as drug delivery systems because their properties can be tailored and they can be easily modified and functionalized, their size and shape can be easily controlled. In this thesis, mainly three polymerbased systems are discussed for drug delivery purpose, namely micelles, nanogels and dendrimers. 


\section{Micelles}

Amphiphilic block copolymers can self-assemble into spherical micelles in aqueous solution. (Cabral, Miyata et al. 2018) In polymeric micelles, the hydrophobic blocks pack into 'hydrophobic core' and the hydrophilic tails interact with water molecules which ensures their excellent colloidal stability.(Kwon and Okano 1996) Hydrophobic drugs can be loaded in the hydrophobic core and thus their aqueous solubility can be substantially increased. (Figure 1a) Micelles have a relatively small size 10$100 \mathrm{~nm}$ which is favorable for long circulation behavior and tumor accumulation exploiting the so-called EPR effect (Maeda, Wu et al. 2000) and tumor penetration. (Cabral, Matsumoto et al. 2011, Yin, Wang et al. 2018) Because of their favorable characteristics, a number of micellar formulations is under clinical evaluation.(Cabral and Kataoka 2014, Varela-Moreira, Shi et al. 2017)

\section{Nanogels}

Nanogels are crosslinked networks of hydrophilic polymers with size in the nanometer range. (Figure 1b) These hydrophilic crosslinked polymers absorb relative high amount of water which contribute to their good biocompatibility. In the network, bioactive molecules can be loaded which can be released due to combination of diffusion and network degradation. (Raemdonck, Demeester et al. 2009) Nanogels offer the possibilities to encapsulate a great variety of therapeutic molecules ranging from small molecular drugs, pharmaceutical peptides, proteins and antigens to nucleic acids-based drugs among which pDNA, siRNA and mRNA. Besides by diffusion and degradation, the release of the loaded compounds can also be engineered to response to a certain triggers such as temperature, light, ultrasound, $\mathrm{pH}$ or reducing agents.(Li, van Nostrum et al. 2017)

\section{Dendrimers}

Dendrimers are nano-sized three dimensional tree-like structures consisting of uniform and highly branched polymers. (Figure 1c) (Patel and Patel 2013) Typical dendrimer structure is composed of a symmetric core, branching units and an outer shell. (Abbasi, Aval et al. 2014) They are monodisperse, and are able to across biologic barriers and have desirable circulation time. (Abbasi, Aval et al. 2014, Kesharwani, Jain et al. 2014) These properties makes them attractive drug delivery systems. However, loading drugs into dendrimers faces major challenges. The reason is that the method used to load drugs into dendrimers is based on physical entrapment which requires the polymers to be sufficiently dense to retain drug under sink condition. Synthesizing dense dendrimers would need multiple reaction steps which may result in defect formation within dense dendrimers.(Svenson 2015) 


\section{Chapter 1}

\section{Liposomes}

Liposomes are artificial mostly spherical bi-layer structures from using cholesterol and phospholipids. (Figure 1d) The size of liposome can vary from $25 \mathrm{~nm}$ to $2.5 \mu \mathrm{m}$. (Akbarzadeh, Rezaei-Sadabady et al. 2013) The circulation time of liposomes depends on size and surface properties, smaller liposomes tend to have longer circulation half-life. Particularly coating of liposomes with hydrophilic and uncharged polymers (mostly PEG) increases circulation time (Akbarzadeh, Rezaei-Sadabady et al. 2013). Hydrophobic drugs can be solubilized in the phospholipid bilayer of liposomes whereas hydrophilic drugs can be entrapped in their aqueous core. And payload encapsulation efficiency is determined by its size and number of bilayers.

\section{Natural nanomedicine systems: Extracellular Vesicles (EVs)}

EVs are nanosized phospholipids bilayered structures released from all domains of life which includes eukaryotes, bacteria, fungi and archaea. (Brown, Wolf et al. 2015) They play important roles in cell-cell communication by functional transfer biological cargoes. (Zaborowski, Balaj et al. 2015) In recent years, EVs are under investigation as a new emerging class of nanomedicine as they can overcome natural barriers in human body, have intrinsic cell targeting properties, and be stable in circulation. (Vader, Mol et al. 2016)

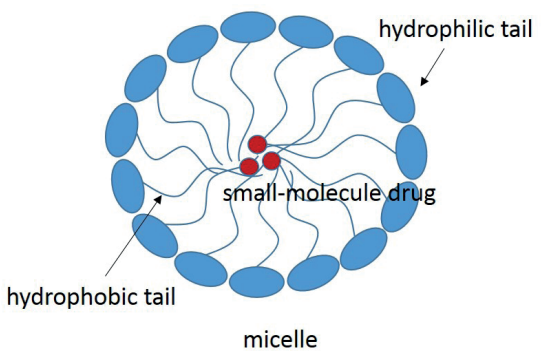

(a)

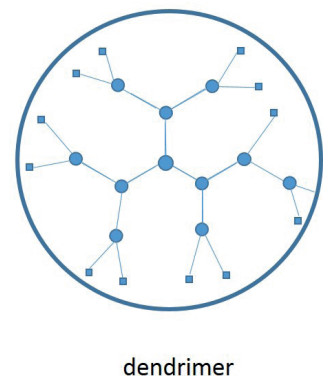

(c)

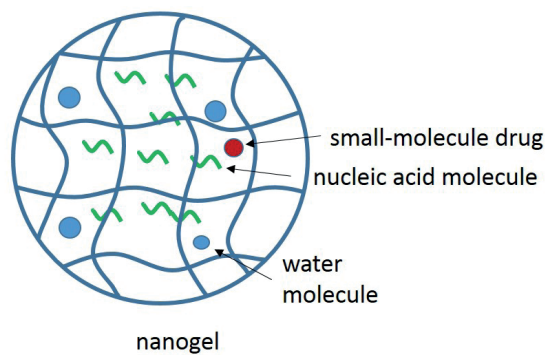

(b)

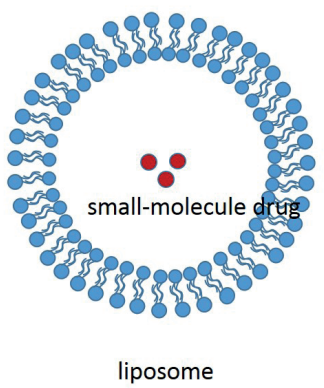

(d)

FIGURE 1. Schematic presentation of synthetic nanomedicine systems studied in this thesis (a) polymeric micelle, (b) nanogel, (c) dendrimer and (d) liposome. 


\section{Nanomedicine systems in markets}

Due to the advantages of nanomedicines over traditional small molecular weight drugs, several synthetic products based on e.g. polymeric micelles and liposomes products have been approved and are summarized in Table 1. No nanogel or dendrimer formulations have been approved yet to date. An EV-based vaccine has been approved by the FDA and is presently used for vaccine purpose.

\section{Outline of this thesis}

In this thesis, two nanomedicine systems are investigated. One is a synthetic system: i.e. a nanogel. The characterization of this novel nanogel is presented, and we also investigated the possibility of using this nanogel for nucleic acid delivery in chapter 2 in both in vitro and in vivo models.

EVs have shown to be an interesting emerging nanomedicine system over the past decade. They can be released by all domains of life which includes eukaryotes, bacteria, fungi and archaea. EVs released by mammalian cells and bacteria are studied in this thesis. We discuss the potential application of mammalian EVs as drug carriers for gene delivery purposes in chapter 3, and discuss the application of bacterial EVs as vaccine candidates in chapter 4.

In addition, we investigated the possibility of using bacterial EVs as 'nano-weapons' for combating bacterial infections. As EVs from Gram-positive bacteria have received less attention than their counterpart from Gram-negative bacteria, in chapter 5, we studied the isolation, characterization and immuno-modulatory aspects of membrane vesicles (MVs) from Gram-positive bacteria Enterococcus faecium E1162 and its isogenic mutants.

To improve the targeting and immunogenicity properties of EVs from both mammalian cells and bacteria, we investigated a post-insertion approach for EVs modification in chapter 6. Finally, a co-culture system which mimic the intestinal environment was set up to evaluate the immunogenicity of OMVs in chapter 7 with the aim to develop an in-vitro platform to study the oral vaccination evaluation of OMVs. In chapter $\mathbf{8}$ the main results of this thesis are summarized and future research directions are discussed. 


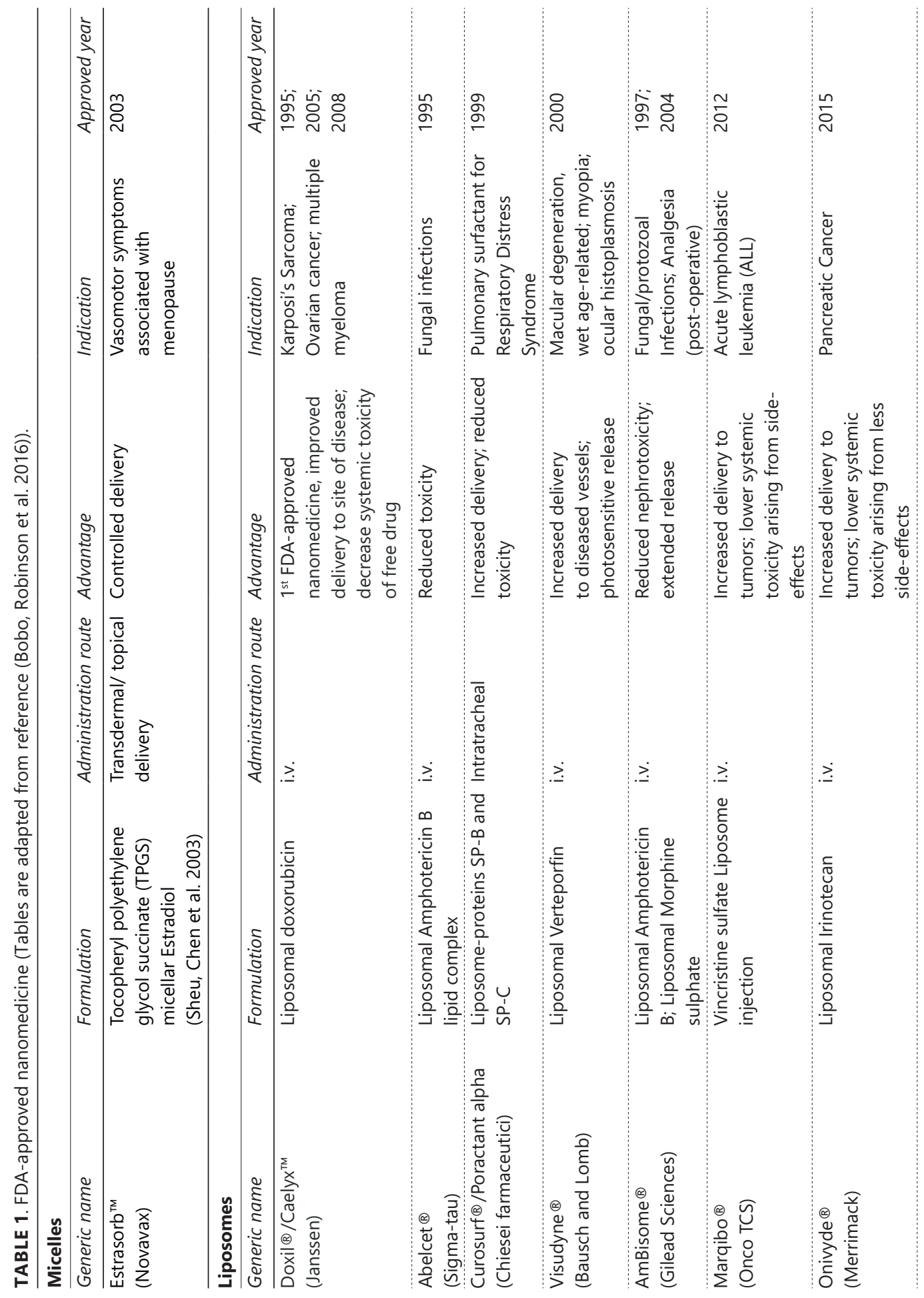


Introduction

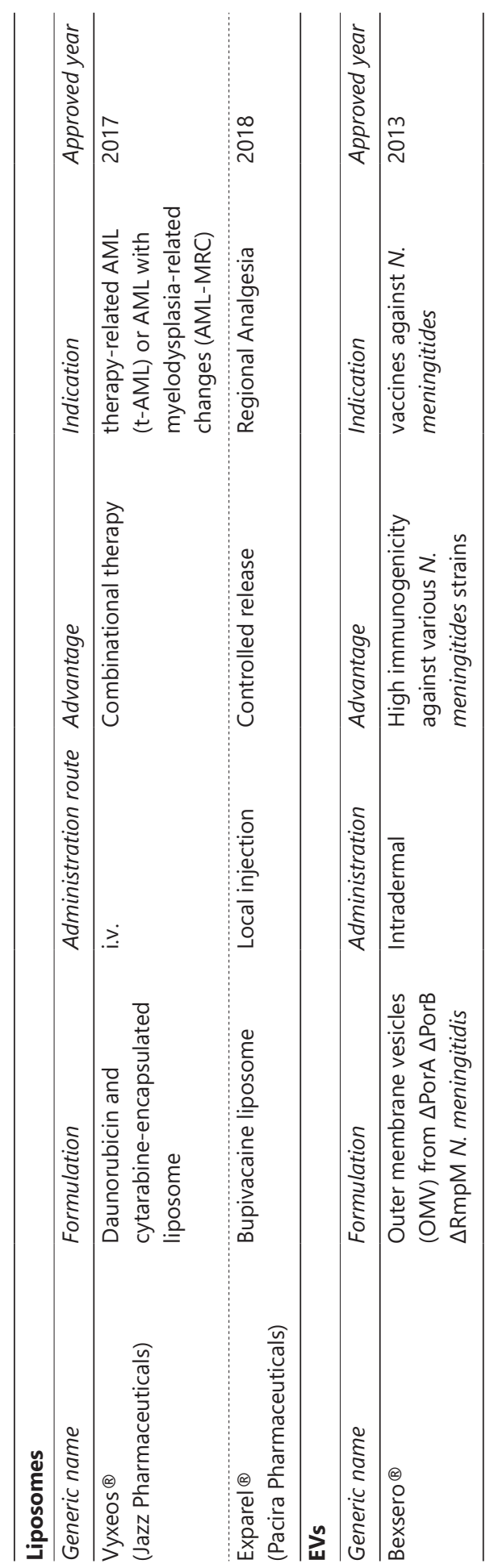




\section{Chapter 1}

\section{References}

Abbasi, E., et al. (2014). "Dendrimers: synthesis, applications, and properties." Nanoscale research letters 9(1): 247.

Akbarzadeh, A., et al. (2013). "Liposome: classification, preparation, and applications." Nanoscale research letters 8(1): 102.

Bobo, D., et al. (2016). "Nanoparticle-based medicines: a review of FDA-approved materials and clinical trials to date." Pharmaceutical research 33(10): 2373-2387.

Brown, L., et al. (2015). "Through the wall: extracellular vesicles in Gram-positive bacteria, mycobacteria and fungi." Nature Reviews Microbiology 13(10): 620-630.

Cabral, H. and K. Kataoka (2014). "Progress of drug-loaded polymeric micelles into clinical studies." Journal of Controlled Release 190: 465-476.

Cabral, H., et al. (2011). "Accumulation of sub-100 nm polymeric micelles in poorly permeable tumours depends on size." Nature nanotechnology 6(12): 815-823.

Cabral, H., et al. (2018). "Block copolymer micelles in nanomedicine applications." Chemical reviews 118(14): 6844-6892.

Deatherage, B. L. and B. T. Cookson (2012). "Membrane vesicle release in bacteria, eukaryotes, and archaea: a conserved yet underappreciated aspect of microbial life." Infection and immunity 80(6): 1948-1957.

Farokhzad, O. C. and R. Langer (2006). "Nanomedicine: developing smarter therapeutic and diagnostic modalities." Advanced drug delivery reviews 58(14): 1456-1459.

Jain, K. K. and K. K. Jain (2008). The handbook of nanomedicine, Springer.

Kesharwani, P., et al. (2014). "Dendrimer as nanocarrier for drug delivery." Progress in Polymer Science 39(2): 268-307.

Kwon, G. S. and T. Okano (1996). "Polymeric micelles as new drug carriers." Advanced drug delivery reviews 21(2): 107-116.

Li, D., et al. (2017). "Nanogels for intracellular delivery of biotherapeutics." Journal of Controlled Release 259: 16-28.

Maeda, H., et al. (2000). "Tumor vascular permeability and the EPR effect in macromolecular therapeutics: a review." Journal of Controlled Release 65(1-2): 271-284.

Patel, H. and P. Patel (2013). "Dendrimer applications-a review." Int J Pharm Bio Sci 4(2): 454-463.

Raemdonck, K., et al. (2009). "Advanced nanogel engineering for drug delivery." Soft Matter 5(4): 707-715.

Sheu, M.-T., et al. (2003). "Influence of micelle solubilization by tocopheryl polyethylene glycol succinate (TPGS) on solubility enhancement and percutaneous penetration of estradiol." Journal of Controlled Release 88(3): 355-368.

Suk, J. S., et al. (2016). "PEGylation as a strategy for improving nanoparticle-based drug and gene delivery." Advanced drug delivery reviews 99: 28-51.

Svenson, S. (2015). "The dendrimer paradox-high medical expectations but poor clinical translation." Chemical Society Reviews 44(12): 4131-4144.

Vader, P., et al. (2016). "Extracellular vesicles for drug delivery." Advanced drug delivery reviews 106: 148-156.

Varela-Moreira, A., et al. (2017). "Clinical application of polymeric micelles for the treatment of cancer." Materials Chemistry Frontiers 1(8): 1485-1501.

Wagner, V., et al. (2006). "The emerging nanomedicine landscape." Nature biotechnology 24(10): 1211-1217.

Yang, X., et al. (2014). "Nanomedicine to overcome cancer multidrug resistance." Current drug metabolism 15(6): 632-649.

Yin, H., et al. (2018). "RNA micelles for the systemic delivery of anti-miRNA for cancer targeting and inhibition without ligand." ACS nano 13(1): 706-717.

Zaborowski, M. P., et al. (2015). "Extracellular vesicles: composition, biological relevance, and methods of study." Bioscience 65(8): 783-797. 


\section{Chapter 2}

\section{Poly(amido amine)-based nanogels for delivery of mRNA}

Linglei Jiang', Zhiyong Lei ${ }^{1,2}$, Pieter Vader ${ }^{1,2}$, Wim E. Hennink ${ }^{3}$, Johan F.J. Engbersen ${ }^{4}$, Raymond M. Schiffelers'

1. CDL Research, Division LAB, Faculty of Medicine, University Medical Center Utrecht, Utrecht University, Utrecht, the Netherlands

2. Experimental Cardiology, Division Heart \& Lungs, University Medical Center Utrecht, Utrecht, the Netherlands

3. Pharmaceutics, Department Pharmaceutical Sciences, Faculty of Science, Utrecht University, Utrecht, the Netherlands

4. 20MED Therapeutics, Enschede, the Netherlands 


\section{Chapter 2}

\section{Abstract}

To enable efficacy of nucleic acid-based therapeutics, delivery vehicles are needed. Here we investigated the use of poly(amidoamine) nanogels with or without poly(ethylene glycol) coating for the delivery of mRNA in vitro and in vivo.

Pre-formed poly(amidoamine)-nanogels efficiently complexed mRNA into $0.1 \mathrm{~mm}$ particles. In the presence of dithiothreitol, particles disassembled and released mRNA. These properties appear important to confer nanoparticle stability outside cells while releasing the mRNA cargo under intracellular reductive conditions. Coating with poly (ethylene glycol) reduced surface charge from $28 \mathrm{mV}$ to $19 \mathrm{mV}$.

In vitro, nanogels were superior in transfecting cells compared to their poly(ethylene glycol)-coated counterparts. Presumably, the poly(ethylene glycol)- efficiently blocks interaction with cells under in vitro conditions, leading to reduced uptake.

Finally, transfection of Cre-recombinase mRNA was tested in Ai9 Cre-recombinase sensitive mice after subcutaneous injection. In contrast to in vitro observations, the poly(ethylene glycol) coated nanogels were superior in the number of cells that were transfected in the subcutaneous injection area, followed by the non-coated nanogel followed by free mRNA which was essentially devoid of activity. Leakage of mRNA expression to other tissues was essentially absent demonstrating the highly localized activity of nanogel-mediated delivery.

Therefore, the coated nanogel represents an attractive class of synthetic delivery system or local delivery of mRNA. 


\section{Introduction}

Nucleic acids offer tremendous opportunities for therapeutic intervention. In diseases where insufficient quantities of functional proteins are made, supplying the genetic information at the DNA or mRNA level can restore protein functions. A recent breakthrough is the approval of Luxturna (voretigene neparvovec-rzyl). This gene therapy product provides a functional copy of the gene retinal pigment epithelium-specific 65 kDa protein (RPE65). Patients suffering from Leber congenital amaurosis lack sufficient enzyme activity of RPE65 leading to blindness. Treatment with the gene restores enzyme function and patients' vision (Russell, Bennett et al. 2017). The frontrunner vaccine approaches for the current COVID-19 pandemic are also nucleic acid therapeutics encoding for exogenous viral proteins to mount an immune response(Ye, Zhong et al. 2020).

Conversely, nucleic acid therapeutics can also be used to block the expression of disease-causing genes. Also here products have been clinically approved: Onpattro (patisiran) (Adams, Gonzalez-Duarte et al. 2018) and Givlaari (givosiran) (Balwani, Sardh et al. 2020). In this approach, small oligonucleotides are used to block expression of specific mRNAs by causing their degradation in a process known as RNA interference (Setten, Rossi et al. 2019).

Despite these clinical successes, the past decades have proven that it is difficult to turn the opportunities of nucleic acid therapeutics into reality. The fact that nucleic acids are larger than conventional therapeutics and bear a strong negative charge and at the same time have an intracellular site of activity are almost mutually exclusive qualities.

The crucial element in nucleic acid therapeutics is therefore a delivery strategy that can overcome the hurdles to the cytoplasm of the target cell. Recently a poly(amido amine) (PAA)-based nanogel (NG) formulation has been developed aiming to address the various challenges from formulation and shelf-life to biological stability and intracellular release of nucleic acid therapeutics (Elzes, Akeroyd et al. 2016).

The core of the polymer is based on a cationic structure that forms an electrostatic complex with negatively charged nucleotides. This can already improve stability of nucleic acids ex vivo as the complex can protect the payload against degradation by nucleases. In vivo this protection remains important but additionally, the complex should remain stable within the complex in vivo environment, and avoid rapid disassembly and clearance. To avoid toxicity-issues that are associated with the use of many cationic compounds in vivo, polymers have been developed that have more benign characteristics. PAAs have peptidomimetic moieties and are easily equipped with functionalities like alcohol and ether moieties during synthesis (Figure 1). 
$\overbrace{\mathrm{R}^{1}}\left[\mathrm{C}_{\mathrm{A}-\mathrm{R}^{2}-\mathrm{A}^{2}}^{\mathrm{O}}\right.$

FIGURE 1. Chemical versatility of polyamido amine-based nanogels, offering site-specific introduction of functional groups (R1-R4), repeating patterns $(a, b)$ and backbone linkages (A).

An attractive PAA is a copolymer of $\mathrm{N}, \mathrm{N}^{\prime}$-bis(acryloyl)cystamine and 4-amino-1butanol. This polymer is degradable under reducing conditions, reducing toxicity, and has shown in vitro transfection efficiency (Vader, van der Aa et al. 2012).

A feature of this PAA-based NG is an inbuilt property to take advantage of the reducing strength of the intracellular environment. Extracellularly, this property would endow the polymer with stability whereas in the reductive environment intracellularly, disulfide exchange and rapid disassembly takes place (Yang, Chen et al. 2006).

Here, we tested qualitatively the in vivo performance of a PAA based nanogel formulation in transfection of reporter mRNAs. Luciferase mRNA was used to measure protein production by the enzyme-mediated conversion of substrate into light. In addition, we used Cre-recombinase mRNA in Cre-recombinase sensitive model systems. These models are based on Cre-Lox site-specific recombination. During this recombination, DNA modification occurs at specific sequences (Lox $P$ sites) in response to successful delivery and translation of the Cre-recombinase mRNA (Ghosh and Van Duyne 2002). By placing a stop codon between loxP sites gene expression of downstream reporter genes can be enabled upon Cre-recombinase mediated excision.

\section{Materials \& methods}

\section{Polymers}

The PAA-based nanogel NG was obtained from 20MED Therapeutics (Enschede, the Netherlands). The nanogel has a cationic core surrounded by a polyamido amine structure composed of $\mathrm{N}, \mathrm{N}^{\prime}$-bis(acryloyl)cystamine and 4-amino-1-butanol. The version with poly(ethylene glycol)-PEGylated version is functionalized with methoxyPEG (Mw 2000). Both PEGylated and uncoated nanogels were dialyzed over a $1 \mathrm{kDa}$ molecular weight cut-off membrane for $24 \mathrm{~h}$, followed by lyophilization until use. 


\section{Preparation}

Polyplexes were prepared by adding equal volumes of mRNA solution $(60 \mu \mathrm{g} / \mathrm{mL}$, Cre-recombinase or luciferase mRNA (Trilink Biotechnologies, San Diego, CA) to $3 \mathrm{mg} / \mathrm{ml}$ nanogel suspension in RNase free water, followed by gentle mixing, to quantitatively load the mRNA. After 15 min incubation, the loaded nanogels were ready for further use. Branched poly(ethylene imine) (PEI) (ISigma Aldrich, St Louis, MI)-based polyplexes were formed by mixing $60 \mathrm{mg} / \mathrm{ml}$ Cre-recombinase mRNA with an equal volume of $45 \mathrm{mg} / \mathrm{ml} \mathrm{PEI}$ in RNase free water on a vortex.

\section{Characterization}

Dynamic light scattering. The hydrodynamic radius of the nanogels was measured by dynamic light scattering (DLS) using a Zetasizer NS90 (Malvern Panalytical, Malvern, UK) equipped with a $4 \mathrm{~mW} \mathrm{He-Ne}$ laser of $633 \mathrm{~nm}$. Samples were diluted in phosphate-buffered saline (PBS) or PBS supplemented with $2.5 \mathrm{mM}$ dithiothreitol (DTT) and scattering was measured at an angle of $173^{\circ}$ at $37^{\circ} \mathrm{C}$ for 10 seconds 10 times. Samples were measured in triplicate.

\section{mRNA}

mRNA encapsulation - Total mRNA concentration was determined using the Quant-It ${ }^{\mathrm{TM}}$ Ribogreen RNA Assay kit (Thermo Fisher Scientific, Waltmham MA) in the presence of $2.5 \mathrm{mM}$ DTT. Unencapsulated mRNA was determined in PBS after centrifugation over a $1 \mathrm{MDa}$ Vivaspin centrifugal concentrator (Sigma-Aldrich, St. Louis, MA). The encapsulation efficiency was then calculated using the following formula: ((RNA total- RNA unencapsulated)/RNA total)*100\%.

mRNA integrity - $360 \mu \mathrm{l}$ mRNA nanogel suspension was incubated with RNase in the presence or absence of $2.5 \mathrm{mM}$ DTT, subsequently, mRNA was isolated using Trizol/ chloroform method (Rio, Ares et al. 2010). mRNA was resuspended in $40 \mu$ l nucleasefree water and immediately used for CDNA synthesis using the iScript CDNA synthesis kit (Bio-Rad, Hercules, CA). PCR was performed using AmpliTaq Gold PCR kit (Thermo Fisher Scientific, Waltham MA) to amplify a 358 bp fragment using forward primer AAACGTTGATGCCGGTGAAC and reverse primer ACCCGGCAAAACAGGTAGTT.

\section{Cell experiments}

Cell culture - COS-7 cells (SV-40 transformed African Green monkey kidney cells) or T47D (engineered with a Cre-recombinase sensitive reporter to switch from red to green fluorescence) cells were seeded in a 96-well CellStar culture plate (Greiner, Kreimsmunster, Austria) at a density of $1 \times 10^{4}$ cells/well in DMEM (Dulbecco's Modified Eagle Medium, Thermo Fisher Scientific, Waltmham MA) supplemented with $10 \%$ fetal bovine serum and $100 \mathrm{U}$ penicillin/100 ug streptomycin/ml (Lonza, 


\section{Chapter 2}

Basel, Switzerland). Cells were incubated overnight at $37{ }^{\circ} \mathrm{C}$ in a humidified $5 \% \mathrm{CO}_{2}$ containing atmosphere. Nanogel-mRNA complexes were diluted in RNase free water to achieve the indicated concentrations. Medium was aspirated from the wells and replaced by $100 \mu \mathrm{L}$ fresh DMEM without serum or antibiotics and up to $12 \mu \mathrm{l}$ of nanogel suspension or DMEM+RNase free water as controls for $4 \mathrm{~h}$. After this, DMEM with serum was added and The plates were incubated for $24 \mathrm{~h}$ or $48 \mathrm{~h}$, after which they were analyzed.

MTT - A $5 \mathrm{mg} / \mathrm{mL}$ of 3-(4,5-dimethylthiazol-2-yl)-2,5-diphenyltetrazolium bromide (MTT) (Thermo Fisher Scientific, Waltham MA) solution was prepared and filtered over a $0.2 \mu \mathrm{m}$ filter. This MTT solution was diluted ten times using cell culture medium. After $48 \mathrm{~h}$ of cell incubation with nanogels, wells were aspirated and $300 \mu \mathrm{l}$ of diluted MTT solution was added. The absorbance of each well was measured at $570 \mathrm{~nm}$ and compared to the absorbance of the untreated controls to quantify the cell viability. All experiments were carried out in triplicate.

Transfection efficiency - $15 \mu \mathrm{l}$ of a $60 \mu \mathrm{g} / \mathrm{ml}$ Cre or luciferase mRNA solution was added to $15 \mu$ of $3 \mathrm{mg} / \mathrm{ml}$ nanogel suspension, mixed and incubated for $15 \mathrm{~min}$ at room temperature. Total volume was brought to $45 \mu \mathrm{l}$ with RNase free water. Cell culture medium was refreshed with $100 \mu \mathrm{l}$ serum- and antibiotics- free medium per well. Between 3-12 $\mu$ of nanogels was added to the well loaded with 60 to $240 \mathrm{ng}$ of mRNA. For Cre-mRNA, green fluorescent cells were visualized by fluorescence microscopy. Luciferase expression was measured using a luciferase assay kit E1500 (Promega Benelux, Leiden, the Netherlands). Twenty $\mu$ l lysis reagent was added per well, $20 \mu \mathrm{l}$ of cell lysate was mixed with $100 \mu \mathrm{l}$ of luciferase assay reagent and luminescence was immediately measured.

\section{Animal experiments}

Cre-mRNA- animal experiments were carried out in Ai9 mice. The Ai9 mouse is a Cre-recombinase sensitive reporter strain with a loxP-flanked stop codon preventing transcription of a red fluorescent protein tdTomato (Liu, Wang et al. 2010). Ai9 mice express tdTomato fluorescence following Cre-mediated recombination upon successful functional transfection with Cre-mRNA. Animals received $2.5 \mu \mathrm{g}$ CremRNA in the free form or formulated as a nanogel subcutaneously in the neck in $125 \mu \mathrm{l}$. Animals were killed one week after injection and tissues were harvested and formalin-fixed and embedded in paraffin. Sections of $10 \mu \mathrm{m}$ were cut using a microtome and subsequently stained with 4',6-diamidino-2-phenylindole (DAPI). Fluorescence microscopy images of the tissues were acquired on a fluorescence microscope. 
Luciferase - Balb/c mice received $5 \mu \mathrm{g}$ luciferase-mRNA in its free form or formulated as a nanogel subcutaneously in the flank in $250 \mu$ l. The suspension was mixed with Evan's blue to mark the position of injection. Animals were killed at $24 \mathrm{~h}$ after injection. Tissue at the site of injection (approx. $100 \mathrm{mg}$ ) was isolated, and snap frozen in liquid nitrogen. The frozen tissue was pulverized to powder by grinding using a mortar and pestle. Subsequently, $500 \mu \mathrm{l}$ of lysis buffer was added and samples were subjected to cycles of vortexing, freezing in liquid nitrogen and thawing. Finally, the samples were centrifuged to pellet cell debris and the supernatant was collected. The luciferase activity in the supernatant was measured as described above for cell culture experiments.

\section{Statistical analyses}

All statistical analyses were performed using Graphpad Prism 8.01. Values are expressed as the mean \pm standard deviation (SD). Two-sided statistical tests were performed in all statistical analyses. Differences were considered statistically significant at $p<0.05$.

\section{Results \& discussion}

\section{Preparation \& Characterization}

Nanogels were loaded with mRNA by coincubation after hydration of the lyophilized nanoparticles. The resulting particles were characterized by DLS. For both nanogel (NG) and PEGylated nanogel (PEG-NG), particles of approximately $80 \mathrm{~nm}$ were obtained (Figure 2). This is an attractive size for transfection as this allows for efficient internalization by cells (Zhao and Stenzel 2018) and is compatible with a variety of administration routes. Particle structure was lost upon addition of 2.5 mM DTT, indicating breakage of intramolecular disulfide bonds and subsequent destabilization of the nanogel particles. This demonstrates that the inbuilt cargo release property appears to be active. Zeta-potential measurements showed a positive surface charge for the NG of $28 \mathrm{mV}$ which was reduced by $9 \mathrm{mV}$ in the presence of the PEG-coating. By moving the plane of shear farther from the polyplex surface, PEG reduces this surface charge. 

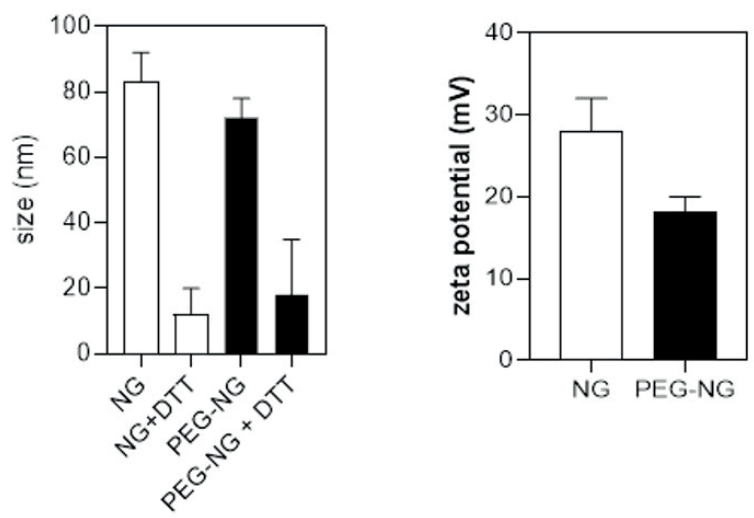

FIGURE 2. Particle size (left panel) and zeta-potential of nanogels. Particle size of NG and PEG-NG was approximately $80 \mathrm{~nm}$. Particle structure was lost upon addition of DTT. Zeta-potential of PEG-NG was 9 $\mathrm{mV}$ lower than for $\mathrm{NG}(28 \mathrm{mV})$. Mean $+/-\mathrm{SD}, \mathrm{n}=3$ preparations.

To assess the mRNA encapsulation efficiency, free RNA obtained after centrifugal concentration of the nanogels was subtracted from the total RNA measured (Figure 3). For the NG encapsulation efficiency was close to $90 \%$ whereas for the PEG-NG it was slightly, but not significantly, lower. Given the substantial costs of mRNA products, it is important to quantitatively capture the nucleic acid, and also because free mRNA can activate the innate immune system (Andries, Mc Cafferty et al. 2015).

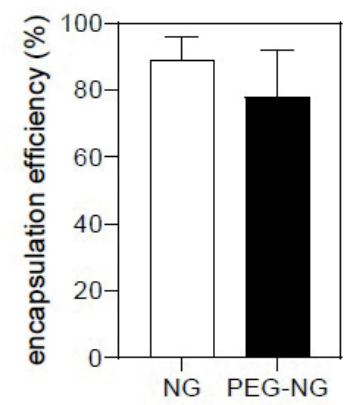

FIGURE 3. Encapsulation efficiency of mRNA in NG and PEG-NG. Mean $+/-$ standard deviation, $n=3$ preparations.

To demonstrate the integrity of the mRNA released from NG and PEG-NG, the released Cre-mRNA was converted to CDNA and amplified using PCR (Figure 4). The 358 bp fragment was only obtained after DTT-mediated destruction of both types of nanogels, indicating the protective environment offered by the nanogels and the release of intact $\mathrm{mRNA}$. 


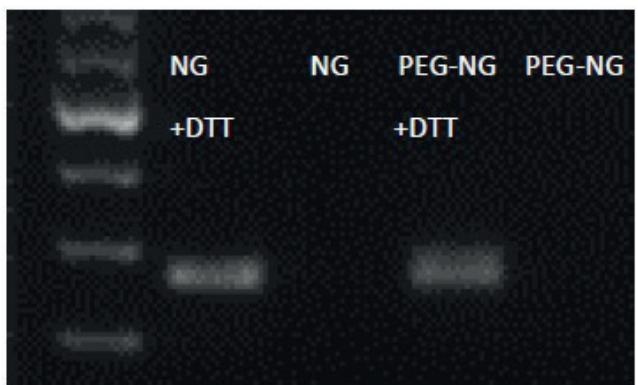

FIGURE 4. Gel electrophoresis of cDNA amplified from mRNA released from the loaded in NG or PEG-NG particles. mRNA could only be amplified after destruction of particle structure with DTT.

\section{In vitro experiments}

\section{Cytotoxicity}

The cytotoxicity of luciferase mRNA as free compound as well as complexed to NG or PEG-NG was compared to that of the known cytotoxic transfection reagent branched PEI (Figure 5). Free mRNA, NG or PEG-NG did not show any demonstrable cytotoxicity at the concentrations tested, whereas bPEI showed significant toxicity, reducing cell viability by approximately $50 \%$ at the highest dose tested.

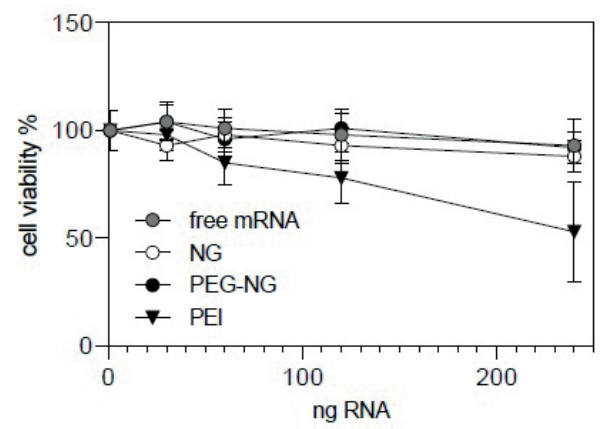

FIGURE 5. Cell viability of COS-7 cells after $72 \mathrm{~h}$ incubations with indicated concentrations of mRNA with or without complexation to NG, PEG-NG or PEl. For reference, at $120 \mathrm{ng}$ mRNA, $90 \mathrm{ng}$ of PEl is added. Because of the reduced charge density NGs are added at 67 times higher concentrations.

\section{In vitro transfection}

The in vitro transfection of the mRNA-loaded nanogels was tested both in a Crerecombinase sensitive reporter cell line T47D using Cre-mRNA (Figure 6) and in COS7 cells transfected with reporter luciferase mRNA (Figure 7). 


\section{Chapter 2}

Free mRNA did not induce a color change in the T47D cells likely due to its degradation in the cell culture medium in combination with insufficient cellular uptake. In contrast, when Cre-mRNA was administered as nanogel formulation, green cells were observed. The number of green cells was related to dose as a higher dose further increased the number of green cells. Interestingly, the PEGylated nanogel formulation with mRNA, was not able to induce green fluorescent protein expression. This might be due to inefficient uptake in vitro as a result of steric stabilization as evidence from the close to neutral zeta-potential of the particles. In vivo a less interactive surface is usually preferred, as it avoids immediate interaction with blood components. Also specificity can be introduced with targeting ligand decoration.
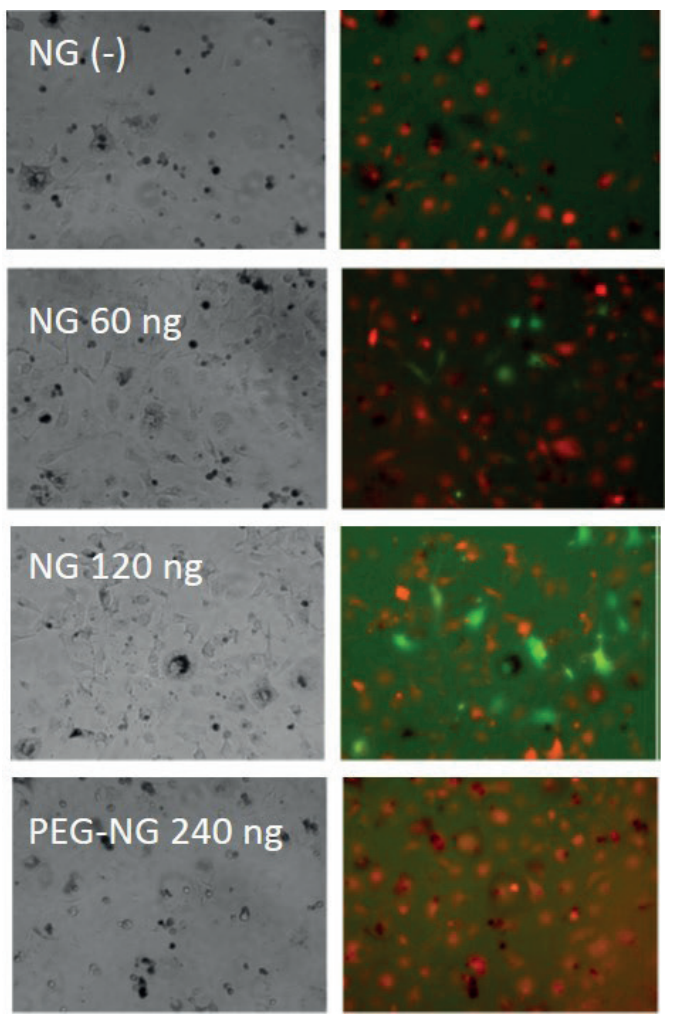

FIGURE 6. Bright field (left) and fluorescence micrographs (right) of Cre-sensitive reporter T47D cells transfected with Cre-recombinase mRNA in the free form or complexed with NG or PEG-NG. Successful transfection with Cre-mRNA induces a color change from red to green fluorescence. Images were taken $48 \mathrm{~h}$ after addition of the mRNA.

The studies using the luciferase expression in COS-7 cells followed the same trend, namely negligible production of luciferase after incubation with free mRNA. Luminescence was almost three orders of magnitude higher for the nanogel delivered 
luciferase mRNA. Again, transfer of PEGylated mRNA was less efficient amounting to $\sim 30$-fold reduced signal as compared to the non-PEGylated version.

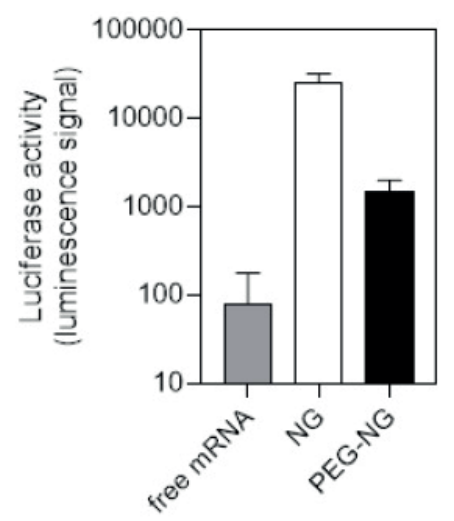

FIGURE 7. Luciferase expression $24 \mathrm{~h}$ after transfection of COS-7 cells with luciferase mRNA in the free form or complexed to NG or PEG-NG (mean +/- standard deviation, $n=3$ ).

\section{In vivo experiments}

Finally, transfection was tested in vivo. Ai9 reporter mice are sensitive to Crerecombinase. Successful Cre-mRNA delivery induces expression of the red fluorescent protein tdTomato. After free mRNA was injected subcutaneously, only very few red cells were observed at the site of injection, which appeared morphologically to be adipocytes (Figure 8). Complexed to NG, more cells were transfected that were morphologically distinct from the cells transfected with free mRNA. In contrast to the in vitro studies, more cells were transfected with PEG-NG compared to NG. Leakage of expression to other organs can be a point of concern for nucleic acid therapy. mRNA transfection was examined for other tissues. Importantly, for all of the treatments and for all of the tissues, negligible numbers of transfected cells were observed. The observation that PEGylated NPs showed higher transfection than their non-pegylated counterparts could point to the fact that positively charged non-pegylated nanogels likely stick TO negatively charged extracellular matrix components and therefore do not reach the cell, whereas PEGylated NPs are more mobile (van den Berg, Oosterhuis et al. 2010).

Finally, transfection of luciferase mRNA after subcutaneous injection was investigated (Figure 9). A similar trend was observed as for Cre-mRNA. Low levels of transfection were noted after injection of free mRNA. Luciferase activity after NG-luciferasemRNA was 6-fold higher. Highest expression was obtained for PEG-NG which was 8-fold higher than for the non-PEGylated NG. 

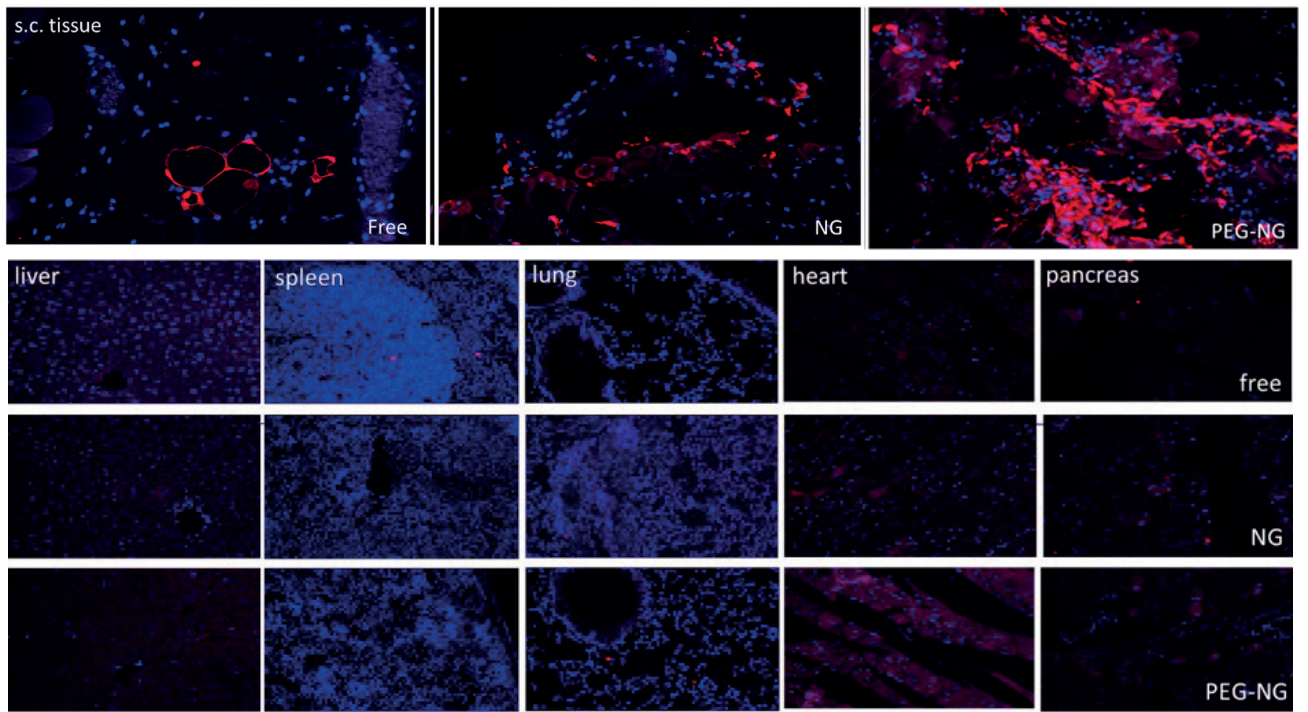

FIGURE 8. Cre-mRNA transfection in Ai9 reporter mice. In these mice successful transfection results in the production of the red fluorescent tdTomato protein in cells. Upper panel shows few red cells at the subcutaneous site of injection for free mRNA (2.5 mg). Complexed to NG, more cells were transfected that were morphologically distinct from the cells transfected with free mRNA. Even more cells were transfected with PEG-NG compared to NG. For all of the treatments and for all of the tissues, negligible numbers of transfected cells were observed.

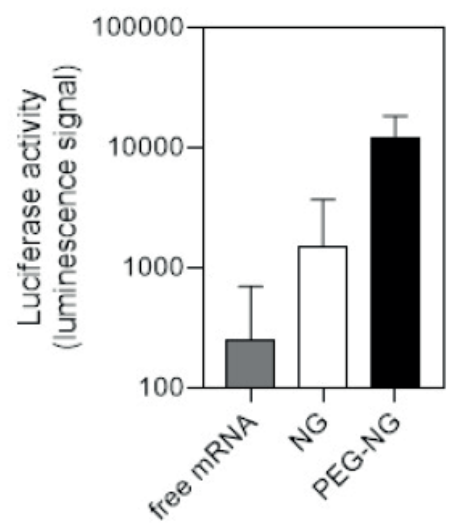

FIGURE 9. Luciferase-mRNA transfection in mice after subcutaneous injection. Free mRNA (2.5. ug) shows low transfection, which is increased for equivalent doses complexed to NG and even higher for PEG-NG.

Taken together the PEG-NG shows attractive properties for in vivo applications at least after local subcutaneous injection. Potential improvements include the use of sheddable PEG-coating to restore an interactive activity after distribution over the tissue (Romberg, Hennink et al. 2008). Alternatively, targeting ligands can be employed to reach specific cells in the area. 


\section{Conflict of interest}

JFJE is founder and shareholder of 20MED Therapeutics (Enschede, The Netherlands). This company has developed the nanogels investigated here.

\section{References}

Adams, D., et al. (2018). "Patisiran, an RNAi therapeutic, for hereditary transthyretin amyloidosis." New England Journal of Medicine 379(1): 11-21.

Andries, O., et al. (2015). "N1-methylpseudouridine-incorporated mRNA outperforms pseudouridineincorporated mRNA by providing enhanced protein expression and reduced immunogenicity in mammalian cell lines and mice." Journal of controlled release 217: 337-344.

Balwani, M., et al. (2020). "Phase 3 Trial of RNAi Therapeutic Givosiran for Acute Intermittent Porphyria." New England Journal of Medicine 382(24): 2289-2301.

Elzes, M. R., et al. (2016). "Disulfide-functional poly (amido amine) s with tunable degradability for gene delivery." Journal of controlled release 244: 357-365.

Ghosh, K. and G. D. Van Duyne (2002). "Cre-loxP biochemistry." Methods 28(3): 374-383.

Liu, Y., et al. (2010). "Isolation of murine bone marrow derived mesenchymal stem cells using Twist2 Cre transgenic mice." Bone 47(5): 916-925.

Rio, D. C., et al. (2010). "Purification of RNA using TRIzol (TRI reagent)." Cold Spring Harbor Protocols 2010(6): pdb. prot5439.

Romberg, B., et al. (2008). "Sheddable coatings for long-circulating nanoparticles." Pharmaceutical research 25(1): 55-71.

Russell, S., et al. (2017). "Efficacy and safety of voretigene neparvovec (AAV2-hRPE65v2) in patients with RPE65-mediated inherited retinal dystrophy: a randomised, controlled, open-label, phase 3 trial." The Lancet 390(10097): 849-860.

Setten, R. L., et al. (2019). "The current state and future directions of RNAi-based therapeutics." Nature Reviews Drug Discovery 18(6): 421-446.

Vader, P., et al. (2012). "Physicochemical and biological evaluation of siRNA polyplexes based on PEGylated poly (amido amine) s." Pharmaceutical research 29(2): 352-361.

van den Berg, J. H., et al. (2010). "Shielding the cationic charge of nanoparticle-formulated dermal DNA vaccines is essential for antigen expression and immunogenicity." Journal of controlled release 141(2): 234-240.

Yang, J., et al. (2006). "Evaluation of disulfide reduction during receptor-mediated endocytosis by using FRET imaging." Proceedings of the National Academy of Sciences 103(37): 13872-13877.

Ye, T., et al. (2020). "Current status of COVID-19 (pre) clinical vaccine development." Angewandte Chemie International Edition.

Zhao, J. and M. H. Stenzel (2018). "Entry of nanoparticles into cells: The importance of nanoparticle properties." Polymer Chemistry 9(3): 259-272. 


\title{
Chapter 3
}

\section{Extracellular vesicles for nucleic acid delivery: progress and prospects for safe RNA-based gene therapy}

\author{
Linglei Jiang, M.Sc. ', Pieter Vader, PhD', \\ Prof. Raymond Michel Schiffelers, PhD'
}

\author{
' Department of Clinical Chemistry and Hematology, University Medical Center Utrecht, Heidelberglaan 100, 3584 CX \\ Utrecht, The Netherlands \\ Corresponding author: \\ Prof. Raymond Michel Schiffelers, PhD \\ Professor of Nanomedicine \\ Laboratory Clinical Chemistry \& Haematology \\ Heidelberglaan 100 \\ 3584 CX Utrecht \\ Room G03.647 UMC Utrecht \\ Tel +31637610587 \\ Tel +31887556512 \\ Fax +31887555418 \\ R.Schiffelers@umcutrecht.nl
}




\section{Chapter 3}

\section{Abstract}

Nucleic acid-based drugs offer a potentially effective tool for treatment of a variety of diseases, including cancer, cardiovascular diseases, neurological disorders, and infectious diseases. However, clinical applications are hindered by instability of RNA molecules in the circulation and lack of efficient vectors that can deliver RNAs to target tissues and into diseased target cells. Synthetic polymer and lipids as well as virus-based vectors are among the most widely explored vehicles for RNA delivery, but clinical progress has been limited as a result of issues related to toxicity, immunogenicity and low efficiency. Most recently, the discovery that extracellular vesicles (EVs) are endogenous RNA carriers which may display better biocompatibility and higher delivery efficiency as compared to synthetic systems, has provided a ray of hope in coping with the delivery dilemma, and EV-based gene therapy has already sparked general interest both in academia and industry.

In this review, the current knowledge on EV biology and their role in cell-cell communication will be summarized. Promises of EVs as drug carriers and recent technologies on tailoring EVs' biological attributes will be included, and preclinical studies in which EVs have shown promise for therapeutic RNA delivery will be discussed. 


\section{Introduction}

mRNAs encode proteins. In vitro transcribed mRNAs are emerging as a potential new drug class. These synthetic mRNAs allow transient expression of proteins, which enables a wide variety of therapeutic opportunities. mRNA-based cancer immunotherapies and infectious disease vaccines are currently clinically being explored. More challenging, however, are potential applications such as mRNA transfection to produce pluripotent stem cells, genome engineering with mRNAs encoding nucleases and in vivo transfection with mRNA to correct protein deficiencies. (Sahin, Karikó et al. 2014)

Small non-coding RNAs are a class of RNA molecules with a length below 200 nucleotides that can regulate gene expression in both eukaryotes and prokaryotes. (Storz, Altuvia et al. 2005, Toledo-Arana, Repoila et al. 2007) Non-coding small RNAs such as siRNA and miRNA offer an attractive avenue to combat various diseases by regulating the expression of genes through RNA interference (RNAi).

The achilles' heel of gene therapy is the delivery issue: naked RNA molecules cannot reach the interior of target cells due to their size, hydrophilicity and negative charge. Strategies utilizing viruses or synthetic polymers and lipids as drug carrier systems are under investigation to address these problems. However, these systems often are not desirable due to their lack of efficacy, toxicity, immunogenicity or non-specificity. (Zhou, Zhou et al. 2016) For example, cationic lipids commonly used in liposome preparations are reported to invoke undesirable immune responses, while cationic polymers exhibit high cytotoxicity.(Lv, Zhang et al. 2006) Harmful immune responses and toxic side effects have also been reported for viral gene delivery vectors.(Thomas, Ehrhardt et al. 2003) Colloidal carriers are being recognized by the immune system as harmful particles, resulting in a hypersensitivity, or infusion reaction, recently described as complement (C) activation-related pseudoallergy (CARPA).(Szebeni 2005)

An emerging new class of biological RNA delivery systems, that is increasingly being investigated, is known as extracellular vesicles (EVs). EVs are nano-sized vesicles with a phospholipid bilayer that are released by cells in organisms from all domains, eukarya, bacteria, and archaea, and are involved in intercellular communication. As EVs are released endogenously, they may offer a safer alternative for gene delivery.

Eukaryotic cell-derived EVs can be subdivided into at least three categories based on their biogenesis: exosomes (originating from multivesicular bodies), microvesicles (formed by direct budding of the plasma membrane) and apoptotic bodies (blebs that are formed when cells undergo apoptosis). 


\section{Chapter 3}

In Gram-negative bacteria, EVs are formed through outward budding from the surface and are known as 'outer membrane vesicles (OMVs)'. OMVs are known to play a role in intercellular as well as inter-species communication. Gram-positive bacteria also release EVs. How these vesicles have penetrated the cell wall to reach the extracellular environment is unknown and still being investigated.(Brown, Wolf et al. 2015) As little is yet known about EVs from Gram-positive bacteria, they will not be covered in this review.

The attractiveness of EVs as drug delivery systems stems from their natural ability to functionally transfer biological molecules, including genetic material.(Raposo and Stoorvogel 2013, Kim, Lee et al. 2015) For example, miR-16-enriched mesenchymal stem cells-derived EVs have been shown to inhibit breast cancer cell growth by down-regulating the expression of the oncogene vascular endothelial growth factor. (Lee, Park et al. 2013) After proinflammatory stimuli, human blood cells have been found to secrete EVs containing miR-150, which was delivered to HMEC-1 endothelial cells and promoted their migration by silencing the c-Myb gene.(Zhang, Liu et al. 2010) This capability of delivering nucleic acids implicates effective overcoming of biological barriers, combined with a potentially superior biocompatibility.(Tibbitt, Dahlman et al. 2016, Zhou, Zhou et al. 2016)

This contribution will focus on eukaryotic-cell derived EVs and Gram-negative bacteria-derived EVs. First, we will discuss molecular compositions and mechanisms involved in their biogenesis, cargo sorting, intercellular trafficking and uptake. Particular emphasis is placed on the diversity of RNA species in EVs. Finally, an overview of current preclinical examples of EV-based gene therapy will be provided and current state-of-the-art technologies in engineering EV will be discussed.

\section{Molecular composition}

\section{Proteins and lipids}

The molecular composition of EVs is dependent on the EV type, the parent cell type, and cell conditions, such as stress or hypoxia.(de Jong, Verhaar et al. 2012, Kucharzewska, Christianson et al. 2013, Li, Lee et al. 2015) Nevertheless, specific proteins have been shown to be enriched in (at least some subtypes of) EVs. Mammalian-cell derived EVs display high levels of tetraspanins (CD9, CD63, and CD81) on their surface, which have been implicated in regulation of the cargo loading process.(Chaput and Théry 2011, Villarroya-Beltri, Baixauli et al. 2014) Integrins and selectins, transmembrane receptors that mediate cell-cell and cell-matrix interactions, are also present, as well as specific lipid binding proteins including lactadherin. These surface proteins appear 
to be important components in the process of EV uptake by cells.(Chaput and Théry 2011)

Besides these surface proteins, proteins which are considered to be important in the biogenesis process can also be detected in EVs, including Alix, Tsg101, clathrin and ubiquitin, as well as proteins involved in membrane trafficking. These include the family of Rab proteins and annexins.(Vlassov, Magdaleno et al. 2012, Colombo, Raposo et al. 2014) Antigen-presenting cell-derived EVs can also display MHC II with potential implications for immune regulatory activity.(Buschow, Van Balkom et al. 2010)

In terms of lipids, EVs are enriched in ceramides, sphingomyelin, phosphatidylserine (PS) and cholesterol compared to the parental cell membranes.(Raposo and Stoorvogel 2013) A more detailed overview of EV composition, including differences between EV subtypes, is provided elsewhere.(Colombo, Raposo et al. 2014)

The molecular composition of Gram-negative bacteria-derived OMVs reflects the composition of the outer membrane, which includes typical outer membrane proteins (OMPs) such as outer-membrane protein A (OmpA), OmpC and OmpF, Cytolysin A (ClyA) and lipoproteins.(Lee, Choi et al. 2008) Some proteins are enriched in OMVs compared to the cellular outer membrane, most notably several toxins are present at high levels in OMVs.(Horstman and Kuehn 2000, Kato, Kowashi et al. 2002) The major lipid class on the outer OMV surface comprises lipopolysaccharides (LPS).(Kim, Kim et al. 2009) In Pseudomonas aeruginosa, for example, OMVs were shown to be almost exclusively composed of B-band LPS, as compared to A-band LPS, whereas this lipid was present at much lower levels in the outer membrane.(Kadurugamuwa and Beveridge 1996) In the OMV lumen, proteins originating from the cellular periplasmic space are primarily recovered, while levels of cytoplasmic proteins are low. The simplified molecular structure and main composition of both mammalian cell-derived EVs and Gram-negative bacteria-derived EVs are depicted in Figure 1.

\section{RNAs}

Besides proteins and lipids, RNA species are also present in EVs. The RNA species in mammalian cell-derived $\mathrm{EV}$ s are well studied given the advances in and accessibility of next-generation sequencing methods. These species include essentially all RNAs of the cell including mRNAs, long noncoding RNAs, miRNA, transfer RNA (tRNA), ribosomal RNA (rRNA), small nuclear RNA (snRNA), small nucleolar RNA (snoRNA), small cytoplasmic RNA, Y-RNA and vault RNA. In addition to full-length RNA molecules, some degraded products are also present in mammalian EVs. However, the relative abundance of the different classes of RNAs differs between EVs and donor cells. For example, vault RNA, SRP-RNA and Y-RNA were found to be enriched 


\section{Chapter 3}

in dendritic cell-derived EVs compared to the parent cells. In addition, the presence of specific RNAs also differs between cells and EVs. Highly abundant cellular miRNAs, such as miR92a-1 and let-7b, were recovered at low levels in EVs, whereas highly abundant EV miRNAs, such as miR-223, miR-142 and miR-93, were found to be expressed at low levels in the originating cells. This suggests that RNA species are selectively packaged into EVs during their formation.(Nolte, Buermans et al. 2012)
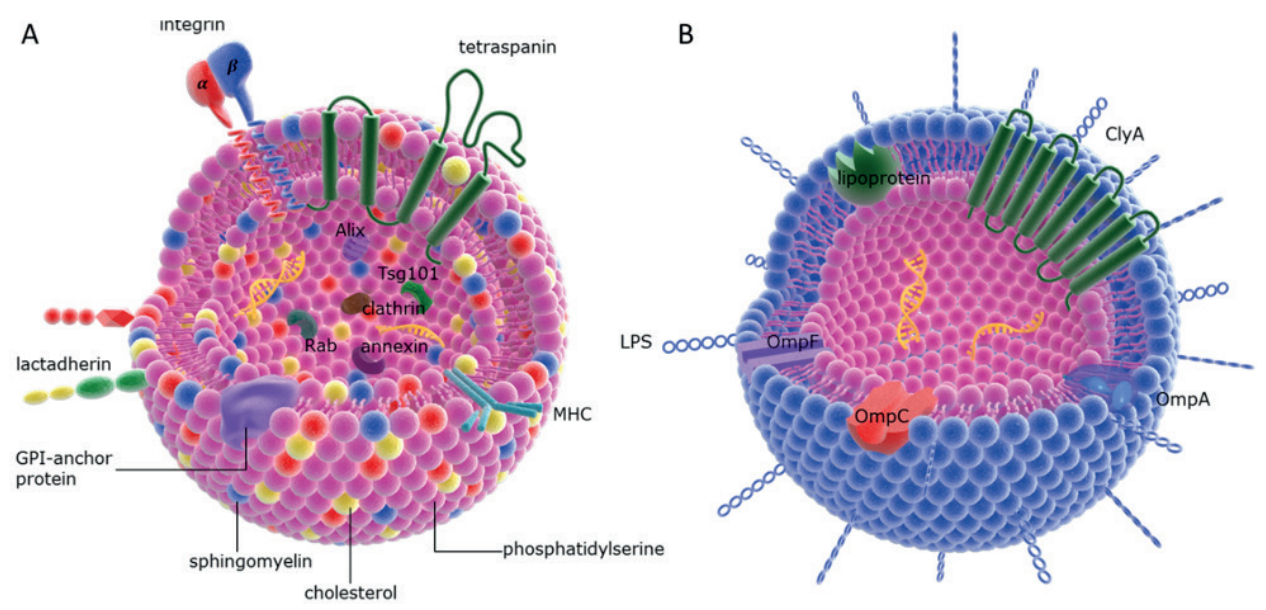

FIGURE 1. Simplified molecular structure and composition of EVs derived from mammalian cells (A) and Gram-negative bacteria (B). Surface protein composition of mammalian cell-derived EVs includes tetraspanins, integrins, MHC molecules, lactadherin, GPI-anchor proteins, associated with EV membrane lipids which include spingomyelin, cholesterol and phosphatidylserine. EV lumen contains RNA and proteins including Alix, Tsg101, annexins, clathrin and Rab proteins. The main composition of membranes from EV Gram-negative bacteria comprises porin proteins such as outer-membrane protein A (OmpA), $\mathrm{OmpC}$, and OmpF, Cytolysin A (ClyA), and lipoproteins.

The most common RNA classes found in EVs and their enrichment in EV are summarized in Table 1. What is striking is the broad distribution of RNA classes recovered in EVs in the various studies. There are many similarities, but also some striking differences. For example, whereas Bellingham et al. noted that rRNA was hardly recovered from their EV samples, it was highly enriched in EVs analyzed by Nolte-'t Hoen et al. This could be attributed to differences in parental cell used for EV production, but could also reflect differences in EV isolation and RNA purification or even sequencing platform.(Witwer, Buzas et al. 2013)

RNA species in bacterial OMVs have been investigated to a lesser extent. It has become clear though that bacteria-derived EVs also contain a large variety of RNA species including tRNA fragments, rRNA fragments, SRP-RNA, 6S RNA and tmRNA. (Ghosal, Upadhyaya et al. 2015) Similar to the findings for mammalian cell- derived EVs, selective packaging of specific RNA species has been noted. 
TABLE 1. RNA species in mammalian cell-derived EVs.

\begin{tabular}{|c|c|c|c|}
\hline Donor cell & RNA species & Enrichment in EVs & Ref. \\
\hline \multirow{15}{*}{$\begin{array}{l}\text { uninfected and } \\
\text { prion-infected } \\
\text { murine neuronal } \\
\text { cells }\end{array}$} & mRNA & & \multirow{15}{*}{$\begin{array}{l}\text { (Bellingham, } \\
\text { Coleman et al. } \\
\text { 2012) }\end{array}$} \\
\hline & mRNA fragment & & \\
\hline & MiRNA & Y & \\
\hline & small nuclear RNA (snRNA) & & \\
\hline & small nucleolar RNA (snoRNA) & & \\
\hline & small cytoplasmic RNA (scRNA) & & \\
\hline & silencing RNA (siRNA) & & \\
\hline & t-RNA & & \\
\hline & YRNA & $\mathrm{Y}$ & \\
\hline & SRP-RNA (or7SL RNA) & Y & \\
\hline & long ncRNA (IncRNA) & Y & \\
\hline & rRNA & & \\
\hline & long intronic ncRNA & & \\
\hline & Piwi-interacting RNA & & \\
\hline & retroviral repeats & & \\
\hline \multirow{10}{*}{$\begin{array}{l}\text { human } \\
\text { endothelial cell } \\
\text { line } 1 \text { (HMEC-1) } \\
\text { cells }\end{array}$} & miRNA & & \multirow{10}{*}{$\begin{array}{l}\text { (van Balkom, } \\
\text { Eisele et al. 2015) }\end{array}$} \\
\hline & miRNA fragment & & \\
\hline & mitochondrial RNA (mtRNA) fragment & Y & \\
\hline & SnORNA & & \\
\hline & YRNA fragment & $\mathrm{Y}$ & \\
\hline & mRNA fragment & Y & \\
\hline & Inc RNA & Y & \\
\hline & small Cajal body-associated RNA (scaRNA) & & \\
\hline & 7SK RNA & & \\
\hline & vault RNA (vRNA) & $\mathrm{Y}$ & \\
\hline \multirow{9}{*}{$\begin{array}{l}\text { dendritic cells } \\
\text { (DCs) }\end{array}$} & t-RNA fragment & $\mathrm{Y}$ & \multirow{9}{*}{$\begin{array}{l}\text { (Nolte, Buermans } \\
\text { et al. 2012) }\end{array}$} \\
\hline & MiRNA & & \\
\hline & t-RNA & & \\
\hline & YRNA & Y & \\
\hline & rRNA & $Y$ & \\
\hline & SRP-RNA & Y & \\
\hline & SRP-RNA fragment & & \\
\hline & vault RNA fragment & $Y$ & \\
\hline & repeat & & \\
\hline
\end{tabular}




\begin{tabular}{|c|c|c|c|}
\hline Donor cell & RNA species & Enrichment in EVs & Ref. \\
\hline \multirow{14}{*}{$\begin{array}{l}\text { MDA-MB-231 } \\
\text { cells }\end{array}$} & rRNA fragment & $Y$ & \multirow{14}{*}{$\begin{array}{l}\text { (Jenjaroenpun, } \\
\text { Kremenska et al. } \\
\text { 2013) }\end{array}$} \\
\hline & SnoRNA & & \\
\hline & SnRNA & & \\
\hline & Mt_tRNA & & \\
\hline & microRNA & & \\
\hline & non-coding RNA & & \\
\hline & guide RNA & & \\
\hline & vault RNA & & \\
\hline & rRNA & & \\
\hline & RNase MRP RNA & & \\
\hline & RNase P RNA & & \\
\hline & Mt_rRNA & & \\
\hline & lincRNA & & \\
\hline & Telomerase RNA & & \\
\hline \multirow[t]{4}{*}{ MCF-7 cells } & tRNA fragment & Y & \multirow{4}{*}{$\begin{array}{l}\text { (Tosar, Gámbaro } \\
\text { et al. 2015) }\end{array}$} \\
\hline & rRNA fragment & $Y$ & \\
\hline & miRNA & & \\
\hline & YRNA fragments & Y & \\
\hline
\end{tabular}

\section{EV biogenesis and cargo sorting mechanism}

EVs are generally classified according to their biogenesis. An improved understanding of the biogenesis and various actors involved can contribute to better understanding of the EV RNA cargo packaging process and help in interpreting the EV-mediated effects and aid in designing EVs with therapeutic RNA cargoes.

Exosomes originate from precursors known as intraluminal vesicles (ILVs) formed in the lumen of multivesicular bodies (MVBs) in the endocytic pathway.(Cicero, Stahl et al. 2015) ILVs are formed by inward budding of the early endosome membrane. During this step, specific cytoplasmic cargo can be loaded, based on cytoplasmic abundance or affinity for the loading machinery. Upon fusion of MVBs with the plasma membrane, ILVs are released by the cell and subsequently referred to as exosomes.(Colombo, Raposo et al. 2014)

The mechanism of MVB biogenesis can be broadly classified into two categories, endosomal sorting complex required for transport (ESCRT) -dependent, and ESCRTindependent. ESCRT consists of five complexes including ESCRT-0, -I, -II and -III and the associated with diverse cellular activities (AAA) ATPase vacuolar protein sortingassociated protein 4 (Vps4) complex.(Henne, Stenmark et al. 2013)

In the ESCRT-dependent mechanism, ESCRT-0 is responsible for sorting transmembrane proteins labeled with ubiquitin into invaginations in the endosomal membrane. This function is mainly accomplished by a phosphatidylinositol 3-phosphate-binding 
protein hepatocyte growth factor-regulated tyrosine kinase substrate (HRS), which, together with signal transducing adaptor molecule (STAM), can recognize the ubiquitinated protein signal.(Colombo, Raposo et al. 2014) ESCRT-I and ESCRT-II also recognize ubiquitinylated cargo and these complexes further regulate membrane bud formation. Activation of ESCRT-III subsequently leads to transient filament growth of this complex which drives vesicle scission and recruitment of de-ubiquitinases that recycle ubiquitin. Finally, the AAA ATPase Vps4 dissociates and recycles the ESCRT machinery.(Kowal, Tkach et al. 2014)

An alternative pathway for MVB biogenesis is ESCRT-independent. For example, through lipids such as phosphatidic acid that can induce a negative membrane curvature, or ceramide that can trigger budding of vesicles into MVBs.(Trajkovic, Hsu et al. 2008) Also tetraspanins, such as CD63, and heat shock proteins, like chaperone HSC70, can partner to induce MVB formation.(Kowal, Tkach et al. 2014)

After formation, MVBs can either fuse with lysosomes, which leads to degradation of their content, or fuse with the plasma membrane which results in exosome release. Both RAB proteins and soluble NSF attachment protein receptor (SNARE) complexes are important in the exosome secretion process. The RAB family consists of more than 60 GTPases, some of which assist in the process of MVB fusion with the target membranes by controlling different steps of intracellular trafficking, including vesicle formation, vesicle mobility and docking to target compartments. (Kowal, Tkach et al. 2014) SNARE proteins contribute after vesicle docking by further facilitating the fusion of MVB and plasma membrane.(Colombo, Raposo et al. 2014)

Microvesicles (MVs), in contrast, are formed via direct shedding from the cell membrane and are considered to be a more heterogeneous vesicle class. Their biogenesis is less well characterized but the process seems to become activated when cells experience stress. The outward budding appears to be enabled by local membrane microdomains and curvature-induced changes in lipid and protein composition, which is complemented by calcium-dependent enzymes like flippase and floppase causing redistribution of phospholipids.(Akers, Gonda et al. 2013)

Several proteins have been shown to play a role but it is unclear whether their role is cell type-specific. ARF6 has been shown to play an important role in the cargo selection and formation of MVs in melanoma cells. Since ARF6 regulates extracellular signal-regulated kinases (ERK) and myosin light-chain kinase (MLCK), actin/myosin dynamics are suggested to be involved in regulating MV shedding.(Muralidharan-Chari, Clancy et al. 2009) Recently, RhoA with several downstream kinases was suggested as a regulator of MV release in breast cancer and cervical carcinoma cells.(Li, Antonyak et al. 2012) In addition, a number of studies have identified calpain, an enzyme capable of cleaving cytoskeletal proteins, to be important in MV biogenesis, in particular in platelets.(Flaumenhaft 2006) 


\section{Chapter 3}

The biogenesis of bacterial OMVs shows similarities to the biogenesis of MVs. Also in bacteria, OMV release is a regulated process primarily triggered by environmental stimuli, in which different stimuli induce release of OMVs of different compositions. (Kadurugamuwa and Beveridge 1995, Schooling and Beveridge 2006, Maredia, Devineni et al. 2012) The release of OMVs requires release of the outer membrane from the peptidoglycan layer. This implies that increased crosslinks between the two limits OMV release, while enzymes, such as endopeptidases, that degrade these crosslinks increase shedding. The reduced crosslinks cause bulging of nanodomains which can be further promoted by accumulation of particular types of LPS and phospholipids and specific LPS-associated molecules. Bacteria have been shown to promote shedding of OMVs in response to temperature(Katsui, Tsuchido et al. 1982), oxidative stress(Thompson, Naidu et al. 1985) and antibiotics.(Kadurugamuwa and Beveridge 1995)

\section{RNA sorting mechanisms}

The abundance of a specific RNA in the cell is an important factor for its loading into EVs. Many RNA species do not appear to show preferential sorting into EVs and simply distribute over both compartments according to statistical chance. Nevertheless, specific sequence motifs appear to promote preferential EV sorting, at least in some cell types. For instance, sequence motifs including 'ACCAGCCU', 'CAGUGAGC', or 'UAAUCCCA' have been shown to serve as cis-acting elements in the mRNA sorting process into EVs.(Batagov, Kuznetsov et al. 2011) Similarly, mRNAs containing a zipcode-like 25-nt sequence with a 'CTGCC' core domain in addition to a miR-1289 binding site in their 3'-untranslated region are more likely to be packaged into EVs derived from human primary glioblastoma cells and melanoma cells-derived EVs.(Bolukbasi, Mizrak et al. 2012) Also, specific motifs have been identified in miRNAs featuring 'GGAG' or 'CCCU' sequences that facilitate targeting into EVs from T lymphoblasts, which appears to be mediated by binding to heterogeneous nuclear ribonucleoprotein A2B1.(Villarroya-Beltri, Gutiérrez-Vázquez et al. 2013) These motifs for sorting miRNA into EVs have been corroborated in gamma-herpesvirus-infected lymphoma cells. Furthermore, miRNAs with 3 '-end uridylated post-transcriptional modifications were found to be preferentially sorted into EVs derived from B-cells. (Koppers-Lalic, Hackenberg et al. 2014) This finding was validated in EVs isolated from human urine. Increasing understanding on sequence motifs that drive RNA loading into EVs may be used when trying to engineer EVs with RNAs of interest for gene therapy purposes.

For OMVs, RNA loading also seems to be regulated but the sorting mechanism has not been clearly elaborated.(Yáñez-Mó, Siljander et al. 2015) 
The critical components in the biogenesis of mammalian cell-derived EVs and Gramnegative bacteria-derived OMVs are schematically depicted in Figure 2 .

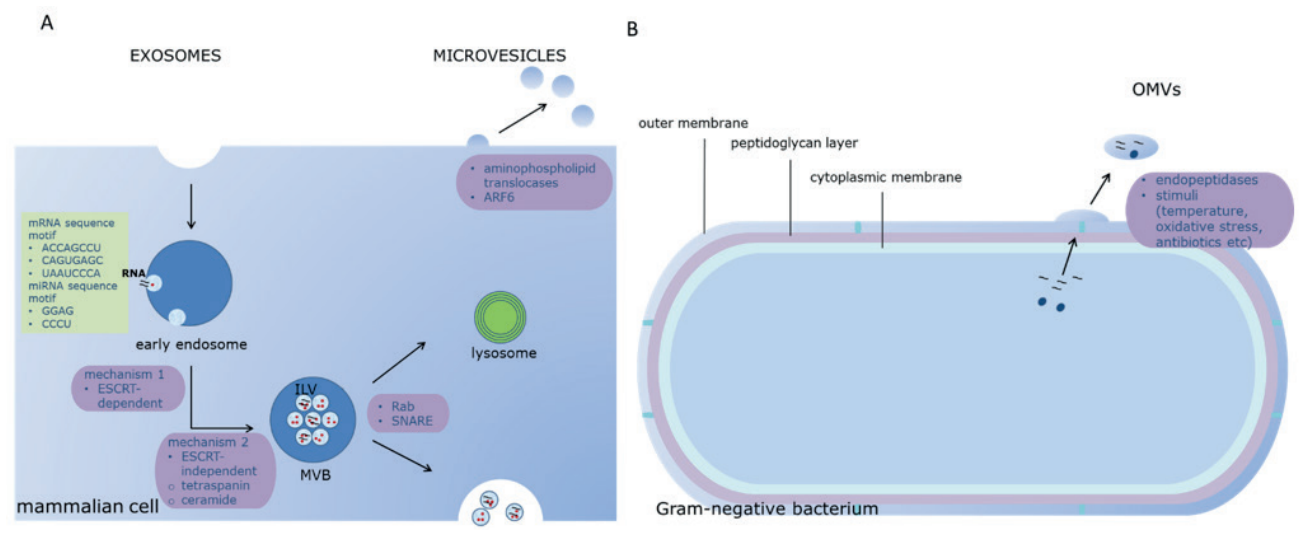

FIGURE 2. Biogenesis mechanism of (A) two subtypes of mammalian cell-derived EVs and (B) Gramnegative bacteria-derived OMVs. Biogenesis of mammalian cell-derived exosomes can be triggered by either ESCRT-dependent or ESCRT-independent pathways. RNAs with specific motifs may show preferential sorting into exosomes. Less is known about the biogenesis process of OMVs, however endopeptidases and stimuli such as temperature, oxidative stress and antibiotics can contribute to OMV budding.

\section{EV-mediated nucleic acid transfer in intra-species, inter-species and inter-kingdom communication}

After release from donor cells, EVs may be taken up by recipient cells via different routes. Various reports have suggested that EVs can either directly fuse with target cells or be internalized via endocytosis.(Morelli, Larregina et al. 2004, Escrevente, Keller et al. 2011, Montecalvo, Larregina et al. 2012) EVs may be endocytosed via clathrin-dependent or clathrin-independent pathways. The clathrin-independent endocytosis pathways include macropinocytosis, phagocytosis and lipid raftmediated endocytosis.(Doherty and McMahon 2009, Mulcahy, Pink et al. 2014)

Bacteria EVs can also enter host mammalian cells, through various pathways, including adhesion-receptor-mediated attachment followed by internalization and/or direct membrane fusion.(Ellis and Kuehn 2010) EV uptake by other bacteria through direct fusion with their outer membrane has also been reported.(Chatterjee and Chaudhuri 2012)

After internalization, EVs can mediate genetic material exchange between cells. The first reports on functional transfer of nucleic acids were shown by Ratajczak et al. and Valadi et al.. Ratajczak and co-workers discovered that embryonic stem 


\section{Chapter 3}

cell-derived EV can transfer mRNA and protein to neighboring cells to reprogram hematopoietic progenitor cells which enhanced their survival.(Ratajczak, Miekus et al. 2006) Valadi et al. demonstrated that mouse mast-cell derived EV mRNA can be transferred to human mast cells and be transcribed into protein.(Valadi, Ekström et al. 2007) Subsequently, Pegtel et al. reported that EVs derived from EBV-infected activated B cells can mediate EBV-miRNA transfer to monocyte-derived DCs which led to down-regulation of miRNA-target genes.(Pegtel, Cosmopoulos et al. 2010) Since then, numerous examples of functional EV-mediated RNA transfer in vitro have been reported, as reviewed elsewhere.(Tetta, Ghigo et al. 2013)

Most recently, several studies have used a Cre-loxP based tracing method to provide evidence for the occurrence and significance of EV-mediated RNA transfer in vivo. For example, Cre mRNA carried by engineered glioma tumor cells was shown to be transferred via EVs to mouse stromal cells and subsequently lead to recombination events, as a results of translation of Cre mRNA into Cre protein.(Ridder, Sevko et al. 2015) In addition, this method enabled the visualization of EV-mediated Cre mRNA transfer between highly metastatic mammary MDA-MB-231 cancer cells and less metastatic T47D cancer cells in vivo, which was shown to change the metastatic behavior of T47D cells.(Zomer, Maynard et al. 2015)

In the prokaryotic kingdom, OMV-mediated genetic transfer between bacteria is important in maintaining their survival. In addition, bacteria-host interactions are important in the pathogenesis of various infectious diseases.(Kulp and Kuehn 2010, Kaparakis-Liaskos and Ferrero 2015)

An additional example of inter-species communication is that OMVs secreted by the nematode parasite can transfer miRNAs, YRNA and nematode Argonaute proteins to suppress the immunity response in the parasite host (mice).(Buck, Coakley et al. 2014) In addition, human cells can communicate with gut microbiota via fecal miRNA-containing EVs, which influences the bacterial gene transcript and its growth status.(Liu, da Cunha et al. 2016)

The capability of EVs to transfer genetic materials makes them important players in intra-species, inter-species and inter-kingdom communication, as the genetic information exchange between donor cells and recipient cells can change the phenotype of the recipient cell.(Valadi, Ekström et al. 2007, Montecalvo, Larregina et al. 2012) This newly discovered way of cell-to-cell communication is increasingly being recognized as of key importance in various physiological as well as pathological processes, as reviewed elsewhere.(Andaloussi, Mäger et al. 2013, Schwechheimer and Kuehn 2015) 


\section{EVs as drug delivery systems for nucleic acids}

Delivery of nucleic acids into target cells is notoriously difficult, especially when considering applications that require systemic administration. The large size and charged character of nucleic acids coupled to their sensitivity to degradation requires a delivery system that protects and delivers the nucleic acid to the intracellular site of activity. The most commonly studied agents for delivery are based on positively charged materials, such as lipids, and polymers, that can establish an electrostatic complex with the negatively charged nucleic acids, thereby forming nanosized particles. In such complexes, the nucleic acid is protected against serum nucleases and is formulated in such a way that allows cellular internalization.

Besides promising developments in using these synthetic systems for nucleic acid delivery, in vitro and in vivo studies have demonstrated that the positive charge of the carrier material is associated with cellular toxicity. In addition, delivery to target cells beyond the liver remains inefficient, hampering widespread use of nucleic acid technology to treat a variety of diseases. This motivates a continuing search for alternative delivery reagents.

EVs are emerging as a novel platform for nucleic acid delivery, and may be used for the delivery of both endogenous and exogenous nucleic acids. For delivery of exogenous nucleic acids, EVs have to be loaded with the desired therapeutic nucleic acid cargo first. However, loading nucleic acids into EVs is not an easy task. Nucleic acids are relatively large and charged molecules which makes it difficult or even impossible to spontaneously penetrate the EV phospholipid bilayer and to be retained inside the lumen of EVs.

Different research groups have investigated loading of nucleic acids into EVs. The majority of these studies focused on siRNA and miRNA (double stranded RNA molecules with 20-25 base pairs). These RNA species are nucleic acids that are relatively short in length and can interfere with the function of targeted mRNAs with a complementary sequence (RNA interference). Successful delivery of these RNAs by EVs would then change the phenotype of receptor cells. The investigated loading methods can be broadly classified into two categories: loading after EV isolation and loading before EV isolation.

\section{Loading after EV isolation}

The first approach comprises loading of EVs after their isolation. In principal, such an approach may allow for loading of all EV types, independent of their cellular or intracellular origin. It is also preferred from a pharmaceutical perspective, as the loading conditions and process can be better controlled. Unfortunately, examples of 


\section{Chapter 3}

successful EV loading with nucleic acids after isolation are few. Methods that have been suggested to be feasible for loading nucleic acids are discussed below.

\section{Simple incubation}

When EVs are incubated with, mainly hydrophobic, cargo of interest, the cargo may interact with the EV phospholipid bilayers driving spontaneous drug loading. Simple incubation was proven to be successful for loading of small molecules such as curcumin, doxorubicin and paclitaxel into EVs, as summarized by Tominaga et al.(Tominaga, Yoshioka et al. 2015) Interestingly, hydrophobically modified siRNA for silencing Huntingtin mRNA was reported to be loaded into glioblastoma U87 cell-derived EVs upon incubation. In this case the modification to increase the hydrophobicity of the nucleic acids enabled them to interact with phospholipid bilayers. (Didiot, Hall et al. 2016) Simple incubation has also been reported to result in the loading of miR-150 into T-cell derived-EVs.(Bryniarski, Ptak et al. 2013) This case for nucleic acid loading is quite different, since the spontaneous transport of nucleic acids over phospholipid membranes is generally regarded as negligible because the charged character of the molecule prevents efficient passage through the hydrophobic part of the bilayer. Although the mechanism behind the apparent loading remains unclear, it is likely that the miRNA is associated at the vesicle surface, which raises questions on the adequateness of miRNA protection from degrading enzymes, as well as the stability of association. It is also not clear whether the observed association is specific for miR-150 and/or T-cell derived EVs.

\section{Electroporation}

Electroporation is a well-established method for cell transfection. Because the membrane of EVs has a similar composition as the cellular membrane, electroporation has been suggested to offer an alternative for actively introducing nucleic acids into EVs. By applying an electrical field in the medium in which EVs are suspended, small pores may temporarily form in the bilayer which theoretically could allow entry of nucleic acid into EVs.(Hood, Scott et al. 2014)

Indeed, a number of groups reported successful EV loading by electroporation. Glyceraldehyde 3-phosphate dehydrogenase (GAPDH) siRNA and (beta-secretase 1) BACE1 siRNA appeared to be loaded into engineered dendritic cell-derived EVs by this method with high efficiency, reaching approximately $25 \%$ of input siRNA. (Alvarez-Erviti, Seow et al. 2011) Similarly, opioid receptor Mu siRNA was reported to be loaded into engineered HEK 293T EVs,(Liu, Li et al. 2015) while mitogenactivated protein kinase 1 (MAPK1) siRNA was introduced into EVs derived from plasma.(Wahlgren, Karlson et al. 2012) Furthermore, KSP siRNA was reported to be loaded into OMVs from a mutant Escherichia coli strain.(Gujrati, Kim et al. 2014) In 
all of these examples, EVs were shown to be capable of delivering the loaded siRNA into recipient cells, resulting in target gene knockdown. These reports also revealed that experimental parameters such as voltage, concentration of EV and cargo, electroporation medium etc. are important for the efficiency of electroporation and these parameters should be tuned to optimize the loading efficiency.

Despite these encouraging results reported for the electroporation method, Kooijmans et al. demonstrated that caution is warranted when interpreting results on loading efficiency. They reported evidence that, at least under certain conditions, siRNA aggregates, formed during the electroporation process, may falsely be interpreted as siRNA loaded into EVs. When this was controlled for, siRNA loading efficiencies turned out to be negligible. They also varied experimental parameters such as voltage, concentration of EVs and cargo, as well as the composition of the electroporation medium, but that did not change the overall loading efficiency. (Kooijmans, Stremersch et al. 2013)

It should also be noted that the EV integrity and nucleic acid structure may be affected by the electroporation procedure, demonstrating the necessity for investigating alternative approaches for EV loading.

\section{Sonication}

Active loading by sonication offers a potential novel avenue to load small RNAs without detectable RNA aggregation and degradation.(Lamichhane, Jeyaram et al. 2016) This sonication method may allow cargo to penetrate EV lipid bilayers by transient pore formation. MCF-7-derived EVs were reported to be successfully loaded with HER-2 siRNA with this approach. However, when applying this method for EV loading, one should bear in mind that experimental parameters such as sonication power, sonication time and the temperature should be well controlled to eliminate undesirable effects on EV structure and biological activity.

\section{Other methods}

A recent report by Haney et al. evaluated various methods for loading of the antioxidant enzyme catalase into EVs derived from macrophages, and demonstrated successful loading using different methods including EV permeabilization with saponin, freeze-thaw cycles, and extrusion.(Haney, Klyachko et al. 2015) However, these methods have not yet been employed for loading of nucleic acids to date. When applying these methods, one should guarantee that the structure and activity of EVs are maintained after treatment. For saponin treatment, it is also critical to ensure that remaining saponin is being removed as saponin can result in toxicity and hemolysis which may affect further experimenting. 


\section{Chapter 3}

\section{Loading before EV isolation}

The second approach, loading before EV isolation, makes use of natural EV loading mechanisms present in cells. After expression or direct transfection of target EV cargo into EV-producing cells, cargo may be sorted into EVs by the endogenous machinery of the cell, although the mechanism involved in cargo sorting remain to be elucidated.(Janas, Janas et al. 2015, Vader, Mol et al. 2016)

Numerous reports have already demonstrated the feasibility of this approach. (Kosaka, Iguchi et al. 2010, Pegtel, Cosmopoulos et al. 2010) For example, HeLa and ascetic fluids-derived EVs were successfully loaded with RAD 51 and RAD 52 siRNA (Lee, Pressman et al. 2009) and Hela-229 cell-derived EVs were loaded with miR$130 \mathrm{~b}$ by this method.(Pan, Yang et al. 2014) Chemically modified miR-143 has been introduced into THP-1 macrophages, and was found to be released in EVs.(Akao, lio et al. 2011) In addition, viruses have been used to generate small RNA-expressing Jurkat and Raji cells, which subsequently produced small RNA-loaded EVs(Rechavi, Erlich et al. 2009)

Although this method provides a straightforward way to produce EVs with desirable cargoes, several pitfalls remain. For example, transfection agents may be difficult to remove and may contaminate EV preparations. In addition, general loading efficiencies are low, dependent on cell- and cargo type, and this method may not be feasible for all RNA species (e.g. expression of miRNAs that affect cell viability or proliferation may also inhibit growth of EV-donor cells).

Together, these reports have demonstrated proof-of-principle for the use of EVs for therapeutic RNA delivery, however efficient cargo loading continues to form a significant hurdle for translation of nucleic-acid loaded EVs to the clinic.

\section{Extracellular vesicle engineering for improving drug delivery properties}

Although EVs seem naturally equipped to transfer nucleic acids to recipient cells, for specific therapeutic applications EVs may be engineered to improve their targeting properties, decrease immunogenicity, or to enhance functionality. For mammalian cell-derived EVs, target cell specificity seems to be largely lacking, especially after systemic administration(Wiklander, Nordin et al. 2015), hence improving EV targeting ability may be advantageous. The targeting properties of EVs may be increased by attaching cell-specific targeting ligands to specific molecular components on the EV surface. For example, fusion proteins of rabies viral glycoprotein (RVG) peptide, 
which specifically binds the acetylcholine receptor expressed on neuronal cells, and EV membrane protein Lamp2b were shown to be expressed on dendritic cell-derived EVs after overexpression in the EV-producing cells, which allowed EV delivery to the brain of mice.(Kumar, Wu et al. 2007, Alvarez-Erviti, Seow et al. 2011) Lamp2b has also been used to display $v$ integrin-specific iRGD (CRGDKGPDC) on EVs, which led to increased EV uptake in breast cancer cells.(Tian, Li et al. 2014) Yet another report showed that HEK 293 cells can be engineered to express the transmembrane domain of platelet-derived growth factor receptor fused to the GE11 peptide (an artificial ligand of EGFR), which subsequently produced modified EVs with GE11 displaying on their surface(Ohno, Takanashi et al. 2013, Ohno, Drummen et al. 2016)

Besides through fusion to surface proteins, targeting moieties have also been expressed on EVs through fusion with glycosylphosphatidylinositol (GPI)-anchor peptides. With such an approach, targeting moieties would localize to GPI-rich lipid rafts in cell and EV membranes. After expression of GPI-anchored, EGFR-targeting nanobodies on EVs derived from Neuro2A cells, modified EVs presented increased binding to EGFR-overexpressing A431 tumor cells.(Kooijmans, Aleza et al. 2016)

A similar approach has been utilized to enhance targeting properties of bacteriaderived EVs. Expression of genetic fusions between an anti-HER2 affibody and the C-terminus of Cytolysin A (ClyA) protein in Escherichia coli resulted in display of the recombinant protein on the cell surface as well as on OMVs. These modified OMVs were shown to be capable of delivering siRNA to HER2-expressing breast cancer cells.(Gujrati, Kim et al. 2014)

For bacteria-derived EVs, safety issues should be considered due to the abundantly present LPS on OMVs. LPS consists of lipid A and a polysaccharide and the latter component can hyperactivate the immune system. Hence, for drug delivery applications, approaches to reduce OMV immunogenicity may be required. The msbB gene which encodes acyltransferase is a well-known virulence factor. A mutation in the msbB gene can result in hypo-acylated LPS which displays reduced virulence. (Somerville, Cassiano et al. 1999) For example, E.coli O157:H7 has been engineered to produce OMVs with mutated lipopolysaccharides by chromosomal tagging of FLAGcontaining DNA constructs within ompA proteins, leading to mutational inactivation of the msbB gene. As a result, penta-acrylated LPS with relatively low endotoxicity will be present on OMVs produced by mutants instead of hexa-acrylated LPS as produced by normal cells.(Kim, Kim et al. 2009) This is analogous to E.coli JM83 and E.coli K12 W3110 strains carrying an msbB mutation, which are also reported to generate LPS with lower endotoxicity.(Somerville Jr, Cassiano et al. 1996) An alternative strategy could be to shorten the o-polysaccharide group to further reduce immunogenicity. (Gujrati, Kim et al. 2014) 


\section{Chapter 3}

Taken together, both mammalian cell-derived EVs and bacteria-derived EVs can be engineered to improve their targeting capacity for specific cell types, to reduce immunogenicity and to provide other functionalities, such as imaging agents. It is critical to choose an engineering site as well as subdomains of this site for consistent fusion results.(Kim, Doody et al. 2008) It should also be noted that engineering may potentially interfere with the natural EV-cell interactions and/or affect intracellular trafficking, and negatively impact EVs' natural biocompatibility. These potential issues will have to be addressed in future studies.

\section{Extracellular vesicle-based therapeutic effects}

Unmodified EVs have been used to achieve therapeutic effects. In these cases, EVs are mostly isolated from stem cells and are thought to contain a cocktail of RNAs and proteins that promote regeneration, reduce inflammation or inhibit tumor growth, as has been reviewed elsewhere.(Burrello, Monticone et al. 2016, Marote, Teixeira et al. 2016) Such EVs have already been applied clinically. EVs derived from mesenchymal stem cells contain many of the immune-modulating factors of the parental cells. In a patient with treatment refractory graft versus host disease, treatment with these EVs reduced pro-inflammatory cytokines and cutaneous and mucosal symptoms of graft versus host-disease and reduced diarrhea.(Kordelas, Rebmann et al. 2014) When utilized for drug delivery purposes, selecting an appropriate EV donor cell type may therefore be of utmost importance. EVs originating from various donor cell types have different molecular compositions and thus may have different biocompatibility and immunogenic properties. Generally speaking, mammalian cell-derived EVs may have better biocompatibility as compared to bacteria-derived EVs, due to bacteria's surface LPS composition which may trigger immune responses. But also EVs of different mammalian cell origins display distinct immunological properties. For example, while EVs derived from stem cells and immature dendritic cells harbor anti-inflammatory properties, EVs produced by mature dendritic cells carry MHC molecules on their surface and elicit potent immune activation.(Yin, Ouyang et al. 2013) In addition, as EVs carry a pool of endogenous cargoes with intrinsic biological activity, their transfer to recipient cells may elicit a complex biological response, with the potential risk of introducing an undesirable phenotype. Special attention should be paid when utilizing tumor-cell derived EVs since these may carry oncogenes causative of cancer progression.

The previous chapter has illustrated that introducing exogenous nucleic acids into EVs is not straightforward but is feasible. Similarly, engineering the surface of EVs to improve targeting or delivery capabilities has been shown to be possible. This provides a toolbox to embark on therapeutic studies with tailored EVs. In this section we provide examples of tailored EV-based therapy in vivo in various models of disease. 
EVs from dendritic cells have been used in delivery of GAPDH siRNA, BACE1 siRNA and -Syn siRNA specifically to mouse neural cells, which can be potentially applied in neurodegenerative disease treatment.(Alvarez-Erviti, Seow et al. 2011) (Cooper, Wiklander et al. 2014)

Exogenous miRNAs miR-124 and miR-145 have been introduced into MSCs via transfection, and these engineered MSCs released EVs containing miR-124 or miR145 , which induced the differentiation of neural cells and increase the expression of glutamate transporter.(Lee, Finniss et al. 2014)

Glioblastoma U87 cell-derived EVs could efficiently be loaded with hydrophobically modified Huntingtin targeting siRNAs (hsiRNAs) upon co-incubation, and hsiRNAcontaining EVs displayed target gene silencing effects after internalization by mouse primary cortical neurons, which suggests that hsiRNAs-loaded EV may be used for treatment of neurodegenerative diseases.(Didiot, Hall et al. 2016)

EV-based gene therapy also appears to show promising effects on treatment of drug addiction. Opioid receptor mu (MOR) siRNA could be packaged into engineered HEK 293T EVs, and these EVs were shown to efficiently deliver siRNA into neuronal cells such as Neuro2A to downregulate MOR expression, which is implicated in drug relapse but also several other central nervous system disorders.(Liu, Li et al. 2015)

EV-based gene therapy has also been applied in the oncology field. Let-7 is a family of miRNAs that have been shown to act as tumor suppressors by reducing expression of oncogenes such as RAS and HMGA2. Therefore, let-7 containing EVs were explored as an antitumor agent. HEK293 cells were modified with a plasmid encoding the EGFR targeting peptide GE11 coupled to the transmembrane domain of platelet-derived growth factor receptor. These cells were also transfected with synthetic let-7a. $1 \mu \mathrm{g}$ of EVs injected intravenously once weekly for 4 weeks induced significant tumor growth inhibition.(Ohno, Takanashi et al. 2013)

In a similar set-up, HEK-293T cells were engineered to express high levels of the suicide gene cytosine deaminase fused to uracil phosphoribosyltransferase. EVs of these cells contained both mRNA and protein of the suicide gene. EVs were directly injected into pre-established nerve sheath tumors in mice. Upon systemic treatment with the prodrug 5 -fluorocytosine, the prodrug was efficiently converted locally into the active drug by cytosine deaminase leading to tumor growth inhibition.(Mizrak, Bolukbasi et al. 2013) Mouse fibroblast L929 cell-derived EVs (L929 EVs) provided an efficient delivery system of exogenous TGF- $\beta 1$ siRNA which exerted inhibitory effect on the growth and metastasis of murine sarcomas 180 cells.(Zhang, Li et al. 2014)

In addition, promising results utilizing EVs as nucleic acid vectors to treat infections(Pan, Ramakrishnaiah et al. 2011), regulate immune responses(Okoye, 


\section{Chapter 3}

Coomes et al. 2014) and obesity(Pan, Yang et al. 2014) have also been reported (as summarized in Table 2).

Although less well investigated, modified bacteria-derived OMVs also show promising therapeutic results by serving as drug carriers for KSP siRNA, leading to target gene silencing and highly significant tumor growth regression in a mouse breast cancer tumor model.(Gujrati, Kim et al. 2014)

\section{Conclusions}

Gene therapy has the potential to be widely used in the treatment of various diseases, including cancer, infection diseases, and central nervous disorders as an effective, personalized therapy approach. However, several hurdles exist when translating this potential to the clinic, including the need for delivery of nucleic acids to the diseased target site. EVs offer an attractive alternative for nucleic acid delivery from a clinical perspective, because of their biocompatibility and safety profile, as compared to more traditional polymer-, lipid- and virus-based vectors. Their versatile molecular composition makes the manipulation of their content and properties feasible. Existing challenges include identifying the right donor cells for EV production, finding an efficient way to load therapeutic nucleic acid into EVs, as well as improving the targeting properties. Efforts to address these problems will help to promote EV applications, towards safe and targeted RNA-based gene therapy in the clinic. 
Extracellular vesicles for nucleic acid delivery

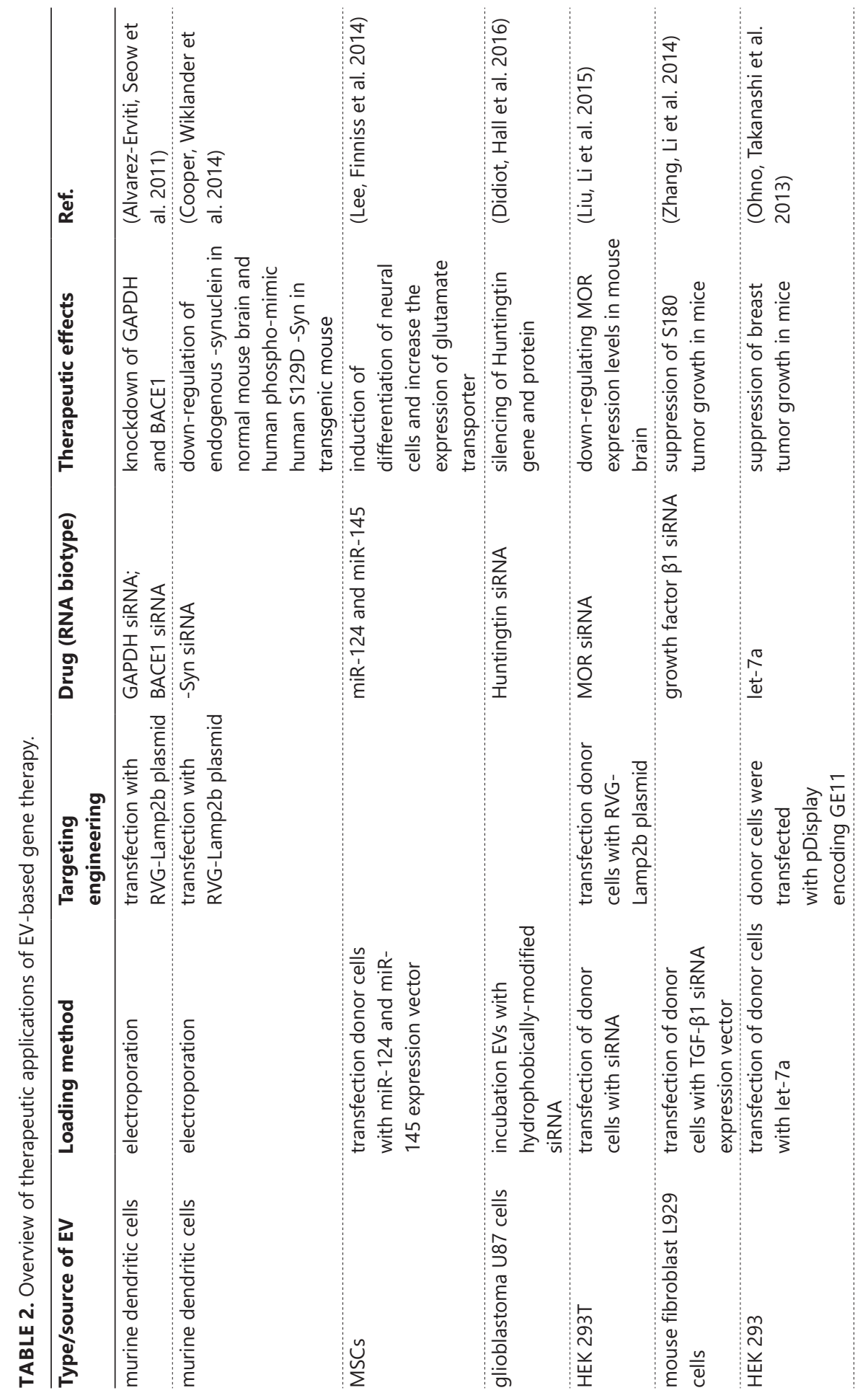




\section{Chapter 3}

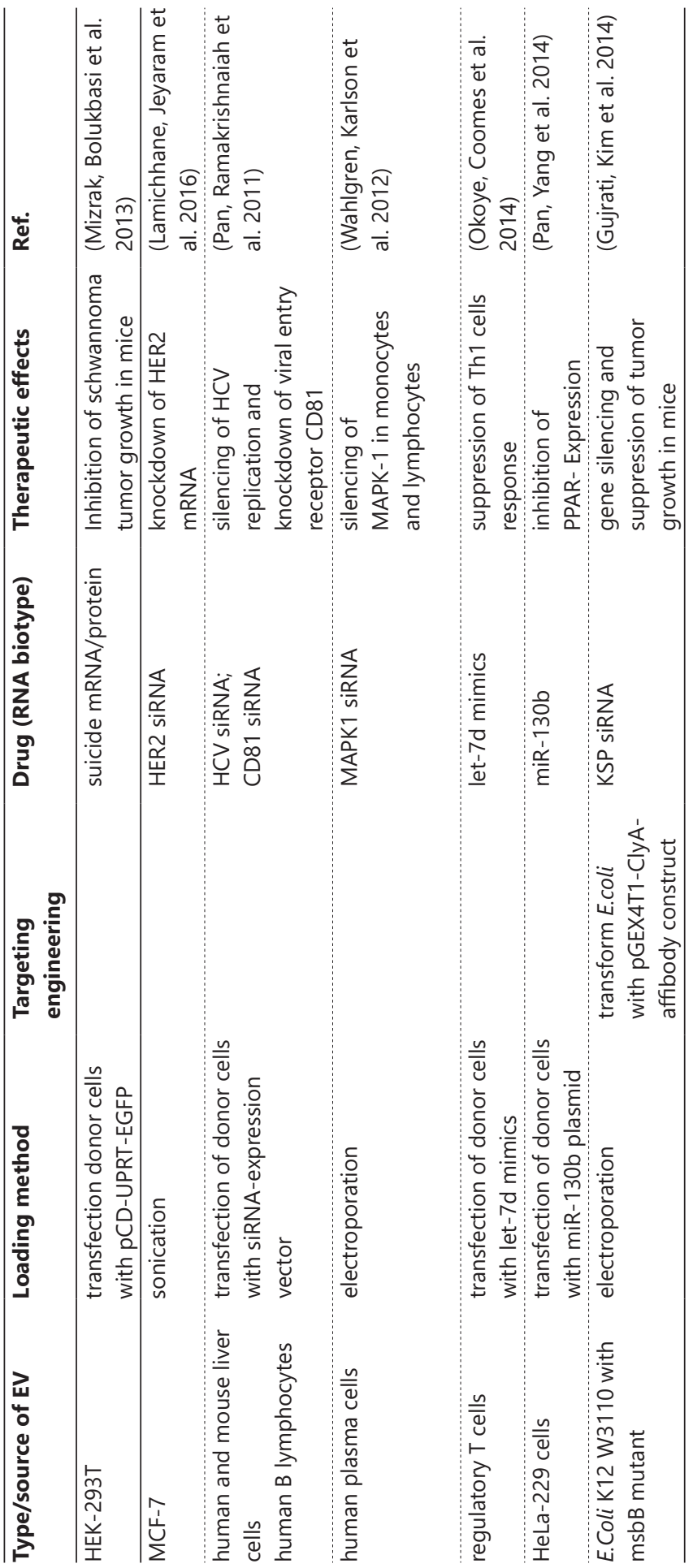




\section{Acknowledgements}

$\sqcup$ acknowledges financial support from the China Scholarship Council (CSC). PV is supported by a VENI Fellowship (\# 13667) from the Netherlands Organisation for Scientific Research (NWO).

\section{Conflicts of interest}

None. 


\section{References}

Akao, Y., et al. (2011). "Microvesicle-mediated RNA molecule delivery system using monocytes/ macrophages." Molecular therapy 19(2): 395-399.

Akers, J. C., et al. (2013). "Biogenesis of extracellular vesicles (EV): exosomes, microvesicles, retrovirus-like vesicles, and apoptotic bodies." Journal of neuro-oncology 113(1): 1-11.

Alvarez-Erviti, L., et al. (2011). "Delivery of siRNA to the mouse brain by systemic injection of targeted exosomes." Nature biotechnology 29(4): 341-345.

Andaloussi, S. E., et al. (2013). "Extracellular vesicles: biology and emerging therapeutic opportunities." Nature Reviews Drug Discovery 12(5): 347-357.

Batagov, A. O., et al. (2011). "Identification of nucleotide patterns enriched in secreted RNAs as putative cis-acting elements targeting them to exosome nano-vesicles." BMC genomics 12(Suppl 3): S18.

Bellingham, S. A., et al. (2012). "Small RNA deep sequencing reveals a distinct miRNA signature released in exosomes from prion-infected neuronal cells." Nucleic acids research: gks832.

Bolukbasi, M. F., et al. (2012). "miR-1289 and "Zipcode"-like sequence enrich mRNAs in microvesicles." Molecular Therapy—Nucleic Acids 1(2): e10.

Brown, L., et al. (2015). "Through the wall: extracellular vesicles in Gram-positive bacteria, mycobacteria and fungi." Nature Reviews Microbiology 13(10): 620-630.

Bryniarski, K., et al. (2013). "Antigen-specific, antibody-coated, exosome-like nanovesicles deliver suppressor T-cell microRNA-150 to effector T cells to inhibit contact sensitivity." Journal of Allergy and Clinical Immunology 132(1): 170-181. e179.

Buck, A. H., et al. (2014). "Exosomes secreted by nematode parasites transfer small RNAs to mammalian cells and modulate innate immunity." Nature communications $\mathbf{5}$.

Burrello, J., et al. (2016). "Stem Cell-Derived Extracellular Vesicles and Immune-Modulation." Frontiers in Cell and Developmental Biology 4.

Buschow, S. I., et al. (2010). "MHC class II-associated proteins in B-cell exosomes and potential functional implications for exosome biogenesis." Immunology and cell biology 88(8): 851-856.

Chaput, N. and C. Théry (2011). Exosomes: immune properties and potential clinical implementations. Seminars in immunopathology, Springer.

Chaput, N. and C. Théry (2011). Exosomes: immune properties and potential clinical implementations. Seminars in immunopathology, Springer.

Chatterjee, S. and K. Chaudhuri (2012). Outer membrane vesicles of bacteria, Springer Science \& Business Media.

Cicero, A. L., et al. (2015). "Extracellular vesicles shuffling intercellular messages: for good or for bad." Current opinion in cell biology 35: 69-77.

Colombo, M., et al. (2014). "Biogenesis, secretion, and intercellular interactions of exosomes and other extracellular vesicles." Annual review of cell and developmental biology 30: 255-289.

Cooper, J. M., et al. (2014). "Systemic exosomal siRNA delivery reduced alpha-synuclein aggregates in brains of transgenic mice." Movement Disorders 29(12): 1476-1485.

de Jong, O. G., et al. (2012). "Cellular stress conditions are reflected in the protein and RNA content of endothelial cell-derived exosomes." Journal of extracellular vesicles $\mathbf{1}$.

Didiot, M.-C., et al. (2016). "Exosome-mediated Delivery of Hydrophobically Modified siRNA for Huntingtin mRNA Silencing." Molecular therapy.

Doherty, G. J. and H. T. McMahon (2009). "Mechanisms of endocytosis." Annual review of biochemistry 78: 857-902.

Ellis, T. N. and M. J. Kuehn (2010). "Virulence and immunomodulatory roles of bacterial outer membrane vesicles." Microbiology and Molecular Biology Reviews 74(1): 81-94.

Escrevente, C., et al. (2011). "Interaction and uptake of exosomes by ovarian cancer cells." $\underline{B M C}$ cancer 11(1): 1. 
Flaumenhaft, R. (2006). "Formation and fate of platelet microparticles." Blood Cells, Molecules, and Diseases 36(2): 182-187.

Ghosal, A., et al. (2015). "The extracellular RNA complement of Escherichia coli." MicrobiologyOpen 4(2): 252-266.

Gujrati, V., et al. (2014). "Bioengineered bacterial outer membrane vesicles as cell-specific drug-delivery vehicles for cancer therapy." ACS nano 8(2): 1525-1537.

Haney, M. J., et al. (2015). "Exosomes as drug delivery vehicles for Parkinson's disease therapy." Journal of Controlled Release 207: 18-30.

Henne, W. M., et al. (2013). "Molecular mechanisms of the membrane sculpting ESCRT pathway." Cold Spring Harbor Perspectives in Biology 5(9): a016766.

Hood, J. L., et al. (2014). "Maximizing exosome colloidal stability following electroporation." Analytical biochemistry 448: 41-49.

Horstman, A. L. and M. J. Kuehn (2000). "Enterotoxigenic Escherichia coli secretes active heat-labile enterotoxin via outer membrane vesicles." Journal of Biological Chemistry 275(17): 12489-12496.

Janas, T., et al. (2015). "Mechanisms of RNA loading into exosomes." FEBS letters 589(13): 1391-1398.

Jenjaroenpun, P., et al. (2013). "Characterization of RNA in exosomes secreted by human breast cancer cell lines using next-generation sequencing." PeerJ 1: e201.

Kadurugamuwa, J. L. and T. J. Beveridge (1995). "Virulence factors are released from Pseudomonas aeruginosa in association with membrane vesicles during normal growth and exposure to gentamicin: a novel mechanism of enzyme secretion." Journal of bacteriology 177(14): 3998-4008.

Kadurugamuwa, J. L. and T. J. Beveridge (1996). "Bacteriolytic effect of membrane vesicles from Pseudomonas aeruginosa on other bacteria including pathogens: conceptually new antibiotics." Journal of bacteriology 178(10): 2767-2774.

Kaparakis-Liaskos, M. and R. L. Ferrero (2015). "Immune modulation by bacterial outer membrane vesicles." Nature Reviews Immunology 15(6): 375-387.

Kato, S., et al. (2002). "Outer membrane-like vesicles secreted by Actinobacillus actinomycetemcomitans are enriched in leukotoxin." Microbial pathogenesis 32(1): 1-13.

Katsui, N., et al. (1982). "Heat-induced blebbing and vesiculation of the outer membrane of Escherichia coli." Journal of bacteriology 151(3): 1523-1531.

Kim, J.-Y., et al. (2008). "Engineered bacterial outer membrane vesicles with enhanced functionality." Journal of molecular biology 380(1): 51-66.

Kim, J. H., et al. (2015). Gram-negative and Gram-positive bacterial extracellular vesicles. Seminars in cell \& developmental biology, Elsevier.

Kim, S.-H., et al. (2009). "Structural modifications of outer membrane vesicles to refine them as vaccine delivery vehicles." Biochimica et Biophysica Acta (BBA)-Biomembranes 1788(10): 2150-2159.

Kooijmans, S. A., et al. (2016). "Display of GPI-anchored anti-EGFR nanobodies on extracellular vesicles promotes tumour cell targeting." Journal of extracellular vesicles $\mathbf{5}$.

Kooijmans, S. A., et al. (2013). "Electroporation-induced siRNA precipitation obscures the efficiency of siRNA loading into extracellular vesicles." Journal of Controlled Release 172(1): 229-238.

Koppers-Lalic, D., et al. (2014). "Nontemplated nucleotide additions distinguish the small RNA composition in cells from exosomes." Cell reports 8(6): 1649-1658.

Kordelas, L., et al. (2014). "MSC-derived exosomes: a novel tool to treat therapy-refractory graft-versushost disease." Leukemia 28(4): 970.

Kosaka, N., et al. (2010). "Secretory mechanisms and intercellular transfer of microRNAs in living cells." Journal of Biological Chemistry 285(23): 17442-17452.

Kowal, J., et al. (2014). "Biogenesis and secretion of exosomes." Current opinion in cell biology 29: 116-125.

Kucharzewska, P., et al. (2013). "Exosomes reflect the hypoxic status of glioma cells and mediate hypoxiadependent activation of vascular cells during tumor development." Proceedings of the National Academy of Sciences 110(18): 7312-7317. 


\section{Chapter 3}

Kulp, A. and M. J. Kuehn (2010). "Biological functions and biogenesis of secreted bacterial outer membrane vesicles." Annual review of microbiology 64: 163.

Kumar, P., et al. (2007). "Transvascular delivery of small interfering RNA to the central nervous system." Nature 448(7149): 39-43.

Lamichhane, T. N., et al. (2016). "Oncogene Knockdown via Active Loading of Small RNAs into Extracellular Vesicles by Sonication." Cellular and Molecular Bioengineering 9(3): 315-324.

Lee, E. Y., et al. (2008). "Proteomics in gram-negative bacterial outer membrane vesicles." Mass Spectrometry Reviews 27(6): 535-555.

Lee, H. K., et al. (2014). "Mesenchymal stem cells deliver exogenous miRNAs to neural cells and induce their differentiation and glutamate transporter expression." Stem cells and development 23(23): 2851-2861.

Lee, J.-K., et al. (2013). "Exosomes derived from mesenchymal stem cells suppress angiogenesis by downregulating VEGF expression in breast cancer cells." PloS one 8(12): e84256.

Lee, Y. S., et al. (2009). "Silencing by small RNAs is linked to endosomal trafficking." Nature cell biology 11(9): 1150-1156.

Li, B., et al. (2012). "RhoA triggers a specific signaling pathway that generates transforming microvesicles in cancer cells." Oncogene 31(45): 4740-4749.

Li, J., et al. (2015). "Serum-free culture alters the quantity and protein composition of neuroblastomaderived extracellular vesicles." Journal of extracellular vesicles 4.

Liu, S., et al. (2016). "The Host Shapes the Gut Microbiota via Fecal MicroRNA." Cell host \& microbe 19(1): 32-43.

Liu, Y., et al. (2015). "Targeted exosome-mediated delivery of opioid receptor Mu siRNA for the treatment of morphine relapse." Scientific reports 5.

Lv, H., et al. (2006). "Toxicity of cationic lipids and cationic polymers in gene delivery." Journal of Controlled Release 114(1): 100-109.

Maredia, R., et al. (2012). "Vesiculation from Pseudomonas aeruginosa under SOS." The Scientific World Journal 2012.

Marote, A., et al. (2016). "MSCs-derived exosomes: cell-secreted nanovesicles with regenerative potential." Frontiers in Pharmacology 7.

Mizrak, A., et al. (2013). "Genetically engineered microvesicles carrying suicide mRNA/protein inhibit schwannoma tumor growth." Molecular therapy 21(1): 101-108.

Montecalvo, A., et al. (2012). "Mechanism of transfer of functional microRNAs between mouse dendritic cells via exosomes." Blood 119(3): 756-766.

Morelli, A. E., et al. (2004). "Endocytosis, intracellular sorting, and processing of exosomes by dendritic cells." Blood 104(10): 3257-3266.

Mulcahy, L. A., et al. (2014). "Routes and mechanisms of extracellular vesicle uptake." Journal of extracellular vesicles 3 .

Muralidharan-Chari, V., et al. (2009). "ARF6-regulated shedding of tumor cell-derived plasma membrane microvesicles." Current Biology 19(22): 1875-1885.

Nolte, E. N., et al. (2012). "Deep sequencing of RNA from immune cell-derived vesicles uncovers the selective incorporation of small non-coding RNA biotypes with potential regulatory functions." Nucleic acids research: gks658.

Ohno, S.-i., et al. (2016). "Focus on Extracellular Vesicles: Development of Extracellular Vesicle-Based Therapeutic Systems." International journal of molecular sciences 17(2): 172.

Ohno, S.-i., et al. (2013). "Systemically injected exosomes targeted to EGFR deliver antitumor microRNA to breast cancer cells." Molecular therapy 21(1): 185-191.

Okoye, I. S., et al. (2014). "MicroRNA-containing T-regulatory-cell-derived exosomes suppress pathogenic T helper 1 cells." Immunity 41(1): 89-103.

Pan, Q., et al. (2011). "Hepatic cell-to-cell transmission of small silencing RNA can extend the therapeutic reach of RNA interference (RNAi)." Gut: gutjnl-2011-300449. 
Pan, S., et al. (2014). "Microvesicle-Shuttled miR-130b Reduces Fat Deposition in Recipient Primary Cultured Porcine Adipocytes by Inhibiting PPAR- $\gamma$ Expression." Journal of cellular physiology 229(5): 631-639.

Pegtel, D. M., et al. (2010). "Functional delivery of viral miRNAs via exosomes." Proceedings of the National Academy of Sciences 107(14): 6328-6333.

Raposo, G. and W. Stoorvogel (2013). "Extracellular vesicles: exosomes, microvesicles, and friends." The Journal of cell biology 200(4): 373-383.

Ratajczak, J., et al. (2006). "Embryonic stem cell-derived microvesicles reprogram hematopoietic progenitors: evidence for horizontal transfer of mRNA and protein delivery." Leukemia 20(5): 847856.

Rechavi, O., et al. (2009). "Cell contact-dependent acquisition of cellular and viral nonautonomously encoded small RNAs." Genes \& development 23(16): 1971-1979.

Ridder, K., et al. (2015). "Extracellular vesicle-mediated transfer of functional RNA in the tumor microenvironment." Oncoimmunology 4(6): e1008371.

Sahin, U., et al. (2014). "mRNA-based therapeutics-developing a new class of drugs." Nature Reviews Drug Discovery 13(10): 759-780.

Schooling, S. R. and T. J. Beveridge (2006). "Membrane vesicles: an overlooked component of the matrices of biofilms." Journal of bacteriology 188(16): 5945-5957.

Schwechheimer, C. and M. J. Kuehn (2015). "Outer-membrane vesicles from Gram-negative bacteria: biogenesis and functions." Nature Reviews Microbiology 13(10): 605-619.

Somerville, J. E., et al. (1999). "Escherichia coli msbB gene as a virulence factor and a therapeutic target." Infection and immunity 67(12): 6583-6590.

Somerville Jr, J. E., et al. (1996). "A novel Escherichia coli lipid A mutant that produces an antiinflammatory lipopolysaccharide." Journal of Clinical Investigation 97(2): 359.

Storz, G., et al. (2005). "An abundance of RNA regulators*." Annu. Rev. Biochem. 74: 199-217.

Szebeni, J. (2005). "Complement activation-related pseudoallergy: a new class of drug-induced acute immune toxicity." Toxicology 216(2): 106-121.

Tetta, C., et al. (2013). "Extracellular vesicles as an emerging mechanism of cell-to-cell communication." Endocrine 44(1): 11-19.

Thomas, C. E., et al. (2003). "Progress and problems with the use of viral vectors for gene therapy." Nature Reviews Genetics 4(5): 346-358.

Thompson, S. S., et al. (1985). "Ultrastructural localization of an extracellular protease in Pseudomonas fragi by using the peroxidase-antiperoxidase reaction." Applied and environmental microbiology 50(4): 1038-1042.

Tian, Y., et al. (2014). "A doxorubicin delivery platform using engineered natural membrane vesicle exosomes for targeted tumor therapy." Biomaterials 35(7): 2383-2390.

Tibbitt, M. W., et al. (2016). "Emerging Frontiers in Drug Delivery." Journal of the American Chemical Society.

Toledo-Arana, A., et al. (2007). "Small noncoding RNAs controlling pathogenesis." Current opinion in microbiology 10(2): 182-188.

Tominaga, N., et al. (2015). "A novel platform for cancer therapy using extracellular vesicles." Advanced drug delivery reviews 95: 50-55.

Tosar, J. P., et al. (2015). "Assessment of small RNA sorting into different extracellular fractions revealed by high-throughput sequencing of breast cell lines." Nucleic acids research: gkv432.

Trajkovic, K., et al. (2008). "Ceramide triggers budding of exosome vesicles into multivesicular endosomes." Science 319(5867): 1244-1247.

Vader, P., et al. (2016). "Extracellular vesicles for drug delivery." Advanced drug delivery reviews.

Valadi, H., et al. (2007). "Exosome-mediated transfer of mRNAs and microRNAs is a novel mechanism of genetic exchange between cells." Nature cell biology 9(6): 654-659. 


\section{Chapter 3}

van Balkom, B. W., et al. (2015). "Quantitative and qualitative analysis of small RNAs in human endothelial cells and exosomes provides insights into localized RNA processing, degradation and sorting." Journal of extracellular vesicles 4.

Villarroya-Beltri, C., et al. (2014). Sorting it out: regulation of exosome loading. Seminars in cancer biology, Elsevier.

Villarroya-Beltri, C., et al. (2013). "Sumoylated hnRNPA2B1 controls the sorting of miRNAs into exosomes through binding to specific motifs." Nature communications 4.

Vlassov, A. V., et al. (2012). "Exosomes: current knowledge of their composition, biological functions, and diagnostic and therapeutic potentials." Biochimica et Biophysica Acta (BBA)-General Subjects 1820 (7): 940-948.

Wahlgren, J., et al. (2012). "Plasma exosomes can deliver exogenous short interfering RNA to monocytes and lymphocytes." Nucleic acids research: gks463.

Wiklander, O. P., et al. (2015). "Extracellular vesicle in vivo biodistribution is determined by cell source, route of administration and targeting." Journal of extracellular vesicles 4.

Witwer, K. W., et al. (2013). "Standardization of sample collection, isolation and analysis methods in extracellular vesicle research." Journal of extracellular vesicles $\mathbf{2}$.

Yáñez-Mó, M., et al. (2015). "Biological properties of extracellular vesicles and their physiological functions." Journal of extracellular vesicles 4.

Yin, W., et al. (2013). "Immature dendritic cell-derived exosomes: a promise subcellular vaccine for autoimmunity." Inflammation 36(1): 232-240.

Zhang, Y., et al. (2014). "Microvesicle-mediated delivery of transforming growth factor $\beta 1$ siRNA for the suppression of tumor growth in mice." Biomaterials 35(14): 4390-4400.

Zhang, Y., et al. (2010). "Secreted monocytic miR-150 enhances targeted endothelial cell migration." Molecular cell 39(1): 133-144.

Zhou, Y., et al. (2016). "Exosome-mediated small RNA delivery for gene therapy." Wiley Interdisciplinary Reviews: RNA.

Zomer, A., et al. (2015). "In vivo imaging reveals extracellular vesicle-mediated phenocopying of metastatic behavior." Cell 161(5): 1046-1057 


\title{
Chapter 4
}

\section{Bacterial membrane vesicles as promising vaccine candidates}

\author{
Linglei Jiang', Michelle Schinkel', Max van Essen', \\ Raymond M. Schiffelers ${ }^{*}$
}

'Laboratory of Clinical Chemistry and Haematology, UMC Utrecht, Utrecht, The Netherlands

Correspondence: Raymond M. Schiffelers (r.schiffelers@umcutrecht.nl) 


\section{Chapter 4}

\section{Abstract}

Both Gram-positive and Gram-negative bacteria can release nano-sized lipid bilayered structures, known as membrane vesicles (MVs). These MVs play an important role in bacterial survival by orchestrating interactions between bacteria and between bacteria and host. The major constituents of MVs are proteins, lipids and nucleic acids. Due to the immunogenicity of the membrane lipids and/or proteins of the $M V s$, in combination with adjuvant danger signals and the repeating patterns on the nanosized surface, MVs can effectively stimulate the innate and adaptive immune system. Since they are non-replicating, they are safer than attenuated vaccines. In addition, by genetic engineering of the host cell, further imporvements to their safety profile, immunogenicity and yield can be achieved. To date, one MV-based vaccine against Neisseria meningitidis (N. meningitidis) serogroup B was approved. Other (engineered) MVs in the pipeline study are mostly in the preclinical phase. 


\section{Introduction}

The first observation of membrane vesicles (MVs) produced by Gram-negative bacteria can be traced back to the 1960's.(Chatterjee and Das 1966) Later on, MVs from Gram-positive bacteria were also confirmed to exist.(Konings, Bisschop et al. 1973, Dorward and Garon 1990), (Lee, Choi et al. 2009) Both Gram-negative and Gram-positive bacteria release MVs under normal culture conditions. Often release is increased under stressed circumstances. MVs were regarded as cellular debris or microscopy artifacts for a long time.(Beveridge 1999) Only recently, the important functional roles of MVs have been reported. These roles include: removal of stress products from the bacterial cells, antimicrobial properties against competitive microorganisms, transfer of antibiotic resistance, contribution to horizontal gene transfer, nutrient acquisition, and nucleation point for the formation of bacterial biofilms. (McBroom and Kuehn 2007, Kulp and Kuehn 2010, Baker, Chen et al. 2014)

The biogenesis of MVs in Gram-negative bacteria and Gram-positive bacteria are different. Understanding the unique bacterial architecture help understand those mechanisms involved. For Gram-negative bacteria, the envelope consists of two membranes, outer membrane (OM) and inner membrane (IM). There is also a thin highly cross-linked peptidoglycan layer (PG) bridging these two layers. One proposed MVs biogenesis mechanism for Gram-negative bacteria is that membrane budding occurs when the OM-PG, OM-PG-IM interaction within envelope temporarily decrease, thus allowing the dissociation of OM and PG.(Deatherage, Lara et al. 2009) It was also noted recently that explosive cell lysis mediated by cryptic prophage endolysin can serve as a new mechanism for the biogenesis of OMVs.(Turnbull, Toyofuku et al. 2016) Gram-positive bacteria contains only one membrane and a thick PG layer, it is also proposed that endolysin can trigger the formation of MVs. (Toyofuku, Nomura et al. 2018)

MVs are $20-250 \mathrm{~nm}$ in size and they exhibit certain similarities in composition to the membrane of the bacteria from which they are released. However, specific proteins such as virulence factors and small RNA are more abundant in MVs than parental bacteriaPorphyromonas gingivalis and $P$. aeruginosa respectively, which implies that a sorting process is involved during biogenesis.(Haurat, Aduse-Opoku et al. 2011, Zhang, Pan et al. 2015, Choi, Um et al. 2017)

MVs offer potential as a platform for vaccines. First of all, a range of antigens and danger signals are displayed on the surface of MVs and encapsulated in the interior, which can stimulate both innate and adaptive immunity. Secondly, MVs are replication defective and therefore safer compared to traditional attenuated bacterial vaccines. However, there are still several challenges to overcome in the application 


\section{Chapter 4}

of MVs, such as unacceptable toxicity of the associated danger signals, poor yield of production and poor immunogenicity.(Baker, Chen et al. 2014)

This review will first focus on current approaches to address the difficulties in the translation of MVs-based vaccines from concept to reality. Secondly, it discusses two examples the current state-of-the-art of MVs-based vaccine: Bexsero ${ }^{\circledR}$ and Bera-V.

\section{Production and isolation of MVs}

MVs are naturally produced by bacteria. Their production can also be induced by treating with detergents such as sodium deoxycholate, enzymatic degradation by lysozyme or mechanical shearing force.(Kulp and Kuehn 2010) The molecular composition of naturally produced MVs differs from induced MVs.(van der Pol, Stork et al. 2015, Gnopo, Watkins et al. 2017)

The workflow for harvesting of MVs consists of cultivation, removal of bacterial cells, concentration, isolation and purification steps. Usually, bacteria are inoculated in a suitable medium to reach their exponential /stationary phase of growth.(Klimentová and Stulík 2015) The compositions of MVs is different for the different growth phases. For example, Acm (a collagen binding adhesin) is detected in MVs isolated from Enterococcus faecium E155 in their stationary-phase, but not in log-phase MVs. The opposite is true for PtsD (Enzyme IID subunit of the phosphotransferase system). (Wagner, Joshi et al. 2018) Usually, a higher yield of MVs is obtained when bacteria are cultured up to the stationary phase, but it runs the risk of reducing sample purity due to contamination with membrane fragments as a result of bacterial lysis.(Wagner, Joshi et al. 2018)

To harvest MVs, one of the critical steps is to remove the intact bacteria via a series of centrifugation and filtration steps. Next, the supernatant/flow-through needs to be concentrated. The most extensively-used methods include ammonium sulphate precipitation methods, ultrafiltration, tangential flow filtration and hollowfiber filtration. Subsequently, density gradient-ultracentrifugation (gUC) and sizeexclusion chromatography (SEC) can be used to further purify MVs from co-isolated contaminants such as large molecular weight soluble proteins, and fragments of flagella, pili, and fimbriae. Density gUC is regarded as golden standard to obtain pure MVs samples, although it is less practical for large scale production. After ultracentrifugation, MVs migrate to the fractions with density similar to their buoyant density. SEC separates MVs based on size rather than density, as a result both purification steps may yield different MV populations. After isolation and purification, absence of live bacteria/spores is confirmed by inoculation in a nutrient-rich liquid medium. 


\section{Characterization and quality control of MVs}

Quality control of EVs has proven to be challenging. To assist the field, the International Society for Extracellular Vesicles published a guideline on the minimal characterization of EVs that should be reported to assert the presence of bona fide vesicles. These characterization criteria are known as the 'minimal information for studies of EVs (MISEV)'. It suggests that three or more MV protein markers should be detected by either western blot, flow cytometry or proteomic analysis. At least two different methods should be used to assess MV population heterogeneity. Transmission electron microscopy (TEM) or atomic force microscopy (AFM) which shows both close-up and wide-field images should be presented in a publication, and the size of EVs should be examined by nanoparticle tracking analyzer (NTA), dynamic light scattering (DLS), or resistive pulse sensing.(Lötvall, Hill et al. 2014) MV yield should be normalized to the number of bacteria by measuring OD value or calculating the Colony Forming Unit (CFU) value. In the MVs product development process, the batch-to-batch variation should also be taken into account. Several approaches can be used examine the variation in protein composition, such as Coomassie blue staining or silver staining of electrophoresis gels. Zeta-potential analysis which measures the surface charge of nanoparticle in solutioncan give information on the aggregation tendency. Lipidomics or lipid staining methods can offer extra information on the lipid compositions among different batches. Regarding the nucleic acid contents next generation sequencing techniques can provide profiles of DNA fragments or RNA species present.

\section{Engineering of $M V s$}

Since MVs are produced from bacteria, toxicity and undesirable immunogenicity remain a point of concern. In addition, their yield is often low and the desired immunogenicity can be insufficient. With genetic engineering, tailoring of the properties of MVs has become possible.

\section{Detoxification}

Lipopolysaccharide (LPS) is one of the major outer membrane constituents of Gramnegative bacteria. It can cause fever, inflammation and septic shock via activation of Toll-like receptor 4 (TLR 4).(Watkins, Rappazzo et al. 2017) Therefore, lowering the LPS content of MVs can be important to limit the inflammatory response. Detergent extraction has been used to reduce the amount of LPS. In addition to LPS, the detergent may reduce/cleave other surface pathogen associated molecular pattern (PAMPs) such as surface-exposed lipoprotein factor $\mathrm{H}$ binding protein 


\section{Chapter 4}

(fHbp), which may be unwanted.(Zariri, Beskers et al. 2016) It is also reported that detergent-extraction methods may increase OMVs' aggregation tendency, which could compromise product quality.(Kaaijk, van Straaten et al. 2013) A detergentfree method, based on EDTA extraction, showed less tendency to aggregate, while maintaining yield and immunogenicity.(Van de Waterbeemd, Streefland et al. 2010)

Genetic engineering is a more elegant method that may be preferred for MV detoxification, various strategies are listed in Table 1 and discussed below. Since the toxicity of LPS is correlated to the degree of acylation(Ranallo, Kaminski et al. 2010), one of the strategies is based on reducing the number of LPS acyl chains. For example, a mutation in the $\operatorname{lp} x \mathrm{~L} 1$ gene (which encodes lipid A biosynthesis lauroyl acyltransferase) in Neisseria meningitides, produces pentacylated LPS. This has reduced toxicity compared to the original hexacylated LPS.(Van de Waterbeemd, Streefland et al. 2010) In a similar approach, inactivating the gene $m s b B$ (also known as $L p x M)$, which encodes the lipid A acyltransferase in Escherichia coli strain O157:H7 and Salmonella enterica serovar Typhimurium, reduced the toxicity of OMVs.(Kim, Kim et al. 2009, Lee, Kim et al. 2009) In E.coli strain BL21, the lipid A structure was modified by deletion of a range of genes that encode enzymes which play important roles in its biosynthesis pathway such as $\operatorname{lp} x L, \operatorname{lp} x M$, pagP, lpxP and eptA. As a consequence, the lipid A precursor lipid $\mathrm{IV}_{\mathrm{A}}$ was incorporated into MVs. This strongly attenuates toxicity, since lipid $\mathrm{IV}_{\mathrm{A}}$ is a human Toll like receptor 4 (TLR4) antagonist. (McBroom, Johnson et al. 2006, Mamat, Wilke et al. 2015, Watkins, Rappazzo et al. 2017) In addition, a msbB/pagP mutant was generated in E. coli $\mathrm{K}-12$ strain W3110, leading to pentacylated lipids and reducing pyrogenicity while maintaining their adjuvant activity on T cells.(Lee, Kim et al. 2011, Lee, Kim et al. 2017)

Another approach to attenuate the toxicity induced by LPS is to modulate the phosphorylation of lipid A. By knocking in the lpxF gene, which encodes lipid A 4'-phosphatase, the 4'-phosphate moiety from lipid IV $\mathrm{A}_{\mathrm{A}}$ and penta-acylated lipid A, can be removed. In the $E$. coli $\mathrm{K}-12$ strain W3110 $\Delta m s b B / \Delta p a g P$ mutant, the additional knock in of $l p x F$, TLR4 activation was further reduced in in-vitro and in-vivo tests.(Lee, Kim et al. 2017)

Studies on attenuating the toxicity of MVs with other target molecules than LPS are few up to date. One good example is the deletion of a global regulator (agr), which regulates the secretion of virulence factors and surface proteins(Traber, Lee et al. 2008). After this deletion, the toxicity of MVs from Staphylococcus aureus was significantly reduced.(Wang, Thompson et al. 2018) 


\section{Antigen display}

Genetic engineering offers an alternative to working with highly pathogenic microorganisms. Heterologous MV-vaccines, where non-pathogenic bacteria are genetically modified to express the antigens of pathogens either on the surface or in the lumen, form a safer alternative.

One of the strategies is to fuse antigens to membrane proteins. Cytolysin A $(\mathrm{ClyA})$, a pore forming transmembrane protein which is enriched in MVs is often used for display. For example, -lactamase, organophosphorous hydrolase, antidigoxin single-chain Fv antibody fragment, and green fluorescent protein (GFP) were coupled to the C-terminus of ClyA. This resulted in the display of all of these antigens on the surface of MVs.(Kim, Doody et al. 2008) GFP fused to ClyA on MVs generated a strong anti-GFP antibody response in mice.(Chen, Osterrieder et al. 2010) MVs can also be engineered using outer membrane protein (OMP). Kim et al. successfully localized a FLAG tag into the lumen of MVs from E.coli by fusing the protein to the $\mathrm{C}$-terminus of the $\beta$-barrel domain of outer membrane protein A (OmpA).(Kim, Kim et al. 2009) Also, antigens from Staphylococcus group A and group B fused to OmpA in E.coli resulted in MVs with staphylococcal antigens, which elicited high antibody titers.(Fantappie, de Santis et al. 2014)

The hemoglobin protease ( $\mathrm{Hbp}$ ) autotransporter-based fusion has also been explored in the MV field. Antigens from Mycobacterium tuberculosis including ESAT6 were fused to Hbp for surface modification of E. coli OMVs.(Jong, Soprova et al. 2012) These antigens together with $\mathrm{Hbp}$ are subsequently released into the extracellular space.(Daleke-Schermerhorn, Felix et al. 2014) It will be explained in more detail in the section on Heterologous vaccines.

Instead of fusing protein domains, another strategy is based on engineering glycotopes onto the lipid A moiety of LPS. With this method, the pathogen-mimetic glycotopes are genetically coupled to the O-antigen polysaccharide moiety of LPS. E.coli expressing the gene cluster encoding glycotopes from the highly virulent Francisella tularensis, resulted in MVs displaying the pathogens glycotope signature. The resulting OMVs elicited the IgG titers and protected mice from an $F$. tularensis challenge.(Chen, Valentine et al. 2016)

So far, it has not clearly been established what the preferred location of antigens associated with MVs is for vaccination: inside the lumen or on the surface. On the surface, it can be expected that exposure to immune cells is more direct to elicit an immune response. However, many studies report luminal loading to be able to induce antibody production.(Gerritzen, Martens et al. 2017) For example, Fantappie and co-worker showed that encapsulated Group A streptococcal (GAS) antigens Slo, spyCEP, Spy0269 inside E.coli MVs, are still able to raise protective 


\section{Chapter 4}

antibodies in a mouse model of lethal GAS infection.(Fantappie, de Santis et al. 2014)

\section{Higher MV production}

The yield of MVs is one of the limiting factors for their application. Since, in principle, the outer membrane of Gram-negative bacteria remains stably connected to the inner layers, shedding of OMVs is necessarily restricted.

To increase yield, hypervesiculating bacteria can be engineered by creating mutations in genes encoding Lpp or Tol-pal proteins that connect membrane to peptidoglycan layer. When the $r m p M$ gene, which belongs to peptidoglycan-associated Lpp family, was deleted in in N. meningitidis, MV production increased.(Van de Waterbeemd, Streefland et al. 2010) In a systematic screening of genes which are important in the vesiculation of an E.coli strain, higher MV production was achieved by knocking out $n l p l$, a gene encoding outer membrane anchored Lpp.(McBroom, Johnson et al. 2006) A study using Shigella boydii in which tolA was deleted showed a $60 \%$ higher vesiculation rate, and, surprisingly, also improved immunogenicity in adult Balb/c mice.(Mitra, Sinha et al. 2016)

For Gram-positive bacteria, the thick outer layer of highly cross-linked peptidoglycan is the main barrier for the biogenesis of MVs. Therefore, higher yield of MVs usually requires degradation of the peptidoglycan layer or reduction of crosslinking. When $S$. aureus was treated with sublethal concentrations of Penicillin G (Pen G) or knocking out peptidoglycan proteins pbp4 or tagO, MV yield increased significantly.(Wang, Thompson et al. 2018)

TABLE 1. Summary of genes used in MV engineering.

\begin{tabular}{|c|c|c|c|}
\hline Objective & Gene & Encoding protein & Ref \\
\hline \multirow[t]{7}{*}{ Detoxification } & $m s b B(L p x M)$ & $\begin{array}{l}\text { Lipid A acyltransferase catalysing final } \\
\text { myristoylation step }\end{array}$ & (Kim, Kim et al. 2009) \\
\hline & $L p x L$ & Lipid A biosynthesis lauroyltransferase & $\begin{array}{l}\text { (Van Der Ley, Steeghs et al. } \\
\text { 2001, Mamat, Wilke et al. 2015) }\end{array}$ \\
\hline & pagP & Lipid A palmitoyltransferase PagP & (Mamat, Wilke et al. 2015) \\
\hline & $L p x P$ & $\begin{array}{l}\text { Lipid A biosynthesis } \\
\text { palmitoleoyltransferase }\end{array}$ & (Mamat, Wilke et al. 2015) \\
\hline & $\triangle e p t A$ & Lipid A phosphoethanolamine transferase & e(Mamat, Wilke et al. 2015) \\
\hline & $\because: p a g L$ & $\begin{array}{l}\text { Lipid A deacylase PagL which removes } \\
\text { 3-hydroxydodecanoic acid moiety at the } \\
\text { 3' position }\end{array}$ & (Zariri, Beskers et al. 2016) \\
\hline & $\because: l p x F$ & $\begin{array}{l}\text { Lipid A 4'-phosphatase which removes } \\
\text { the } 4 \text { '-phosphate moiety from lipid IV(A) } \\
\text { and pentaacylated lipid A }\end{array}$ & (Lee, Kim et al. 2017) \\
\hline
\end{tabular}




\begin{tabular}{|c|c|c|c|}
\hline \multirow[t]{4}{*}{ Antigen display } & OmpA & Outer membrane protein $A$ & $\begin{array}{l}\text { (Kim, Kim et al. 2009, Fantap- } \\
\text { pie, de Santis et al. 2014) }\end{array}$ \\
\hline & ClyA & Cytolysin A & $\begin{array}{l}\text { (Kim, Doody et al. 2008, Chen, } \\
\text { Osterrieder et al. 2010, Gujrati, } \\
\text { Kim et al. 2014) }\end{array}$ \\
\hline & $\mathrm{Hbp}$ & Hemoglobin protease & $\begin{array}{l}\text { (Jong, Soprova et al. 2012, } \\
\text { Daleke-Schermerhorn, } \\
\text { Felix et al. 2014, Kuipers, } \\
\text { Daleke-Schermerhorn et al. } \\
\text { 2015) }\end{array}$ \\
\hline & Glycotopes & $\begin{array}{l}\text { Gene cluster for synthesis of } F \text {. tularensis } \\
\text { Schu S4 O-antigen polysaccharide }\end{array}$ & (Chen, Valentine et al. 2016) \\
\hline \multirow[t]{6}{*}{ Increase yield } & rmpM & Protein links OM and peptidoglycan & $\begin{array}{l}\text { (Van de Waterbeemd, } \\
\text { Streefland et al. 2010) }\end{array}$ \\
\hline & $n l p l$ & OM-anchored Lpp & $\begin{array}{l}\text { (McBroom, Johnson et al. } \\
\text { 2006) }\end{array}$ \\
\hline & TolR & Tol-pal complex & (Mitra, Sinha et al. 2016) \\
\hline & TolA & Tol-pal complex & $\begin{array}{l}\text { (Daleke-Schermerhorn, Felix et } \\
\text { al. 2014) }\end{array}$ \\
\hline & $\begin{array}{l}\text { pbp4 } \\
\text { (Gram-pos- } \\
\text { itive) }\end{array}$ & Penicillin-binding protein 4 & (Wang, Thompson et al. 2018) \\
\hline & $\begin{array}{l}\text { tagO } \\
\text { (Gram-pos- } \\
\text { itive) }\end{array}$ & $\begin{array}{l}\mathrm{N} \text {-acetylglucosamine-phosphate } \\
\text { transferase enzyme }\end{array}$ & (Wang, Thompson et al. 2018) \\
\hline
\end{tabular}

\section{$O M V$ vaccines against meningococci}

Outbreaks of $N$. meningitides caused signficiant morbidity and mortality. There are 12 different $N$. meningitidis serogroups which are classified based on the type of capsular polysaccharide. Of these, six serogroups $A, B, C, X, Y$ and $W$ are the main subtypes contributing to global meningococcal disease.(Bai, Findlow et al. 2011) For serogroup A, C, Y and W, a capsular polysaccharide vaccine has been developed. Since capsular polysaccharides from serogroup $B$ are similar to components of human neural cells, there is a risk of an autoimmune response.(Bai, Findlow et al. 2011) For this particular subtype OMV-based vaccine serves as an effective therapeutic alternative and a good example of the current state of the art.

The first monovalent OMV-based vaccines, each directed at one particular strain of meningococci type $B$ include the MenBvac ${ }^{\circledR}$, VA-Mengoc-BC ${ }^{\circledR}$ and MeNZB ${ }^{\circledR}$. In these products, serosubtype outer membrane protein Porin $A(\operatorname{Por} A)$ is the dominant antigen. Other antigens include Porin $B$ (Por $B)$, reduction modifiable protein (Rmp), and Opc A, a protein involved in bacterial invasion.(Holst, Feiring et al. 2005) 


\section{Chapter 4}

MenBvac ${ }^{\circledR}$ is a first-generation OMV-based vaccine developed in Norway in 19841986 in a period when serogroup B meningococci were highly prevalent in Northern Europe with $\sim 10 \%$ fatality in infected patients.(Bjune, Hoiby et al. 1991) The OMVs were isolated from wild-type strain 44/76-SL by a deoxycholate extraction method. The adjuvant in this formulation is aluminum hydroxide. Other compositions include OM protein, thiomersalate and sucrose.(Bjune, Hoiby et al. 1991) In 1991, a clinical study in healthy volunteers appeared promising.(Høiby, Rosenqvist et al. 1990) However, in the subsequent large-scale randomised trial, the formulation failed to protect $40 \%$ of the vaccinated volunteers.(Bjune, Hoiby et al. 1991) This product was also used in the control of an $N$. meningitides epidemic in France starting in 2006,(Caron, Du Chatelet et al. 2011) Studies showed that children of 1-5 year-old immunized with MenBvac ${ }^{\circledR}$, showed serum bactericidal activity in $84.1 \%$ of children after 6 weeks, while this was 10.8\% before immunization.(Caron, Delbos et al. 2012)

VA-Mengoc-BC ${ }^{\circledR}$ was developed in 1987-1989 in Brazil.(Pérez and Meneses 1999) The formulation consists of OMVs isolated from wild-type strain CU-385, OMPs from serogroup B meningococci, polysaccharide from serogroup C meningcocci, LPS, aluminium hydroxide, and sodium thiomersalate.(Sierra, Campa et al. 1991) It showed $83 \%$ efficacy in a clinical trial in Cuba, eliciting long-lasting and high antibody production.(Sierra, Campa et al. 1991) VA-Mengoc-BC ${ }^{\circledR}$ is used in the public vaccination scheme.(Sierra, Campa et al. 1991)

MeNZB ${ }^{\circledR}$ was developed in New Zealand. It is based on OMVs isolated from the NZ98/554 strain which caused an epidemic in New Zealand around 2000. This OMVbased vaccine induced a 4 -fold rise in MenB-antibody titers in $96 \%$ of adults, and $\sim 75 \%$ of children, toddlers, and infants.(Oster, Lennon et al. 2005) It appears a safe and efficient vaccine for the control of this specific serotype of meningococci.(Oster, Lennon et al. 2005)

Though these meningococcal OMV vaccines have shown promise to control MenB outbreaks in different regions, they do not offer broad protection. Since the major antigen Por $A$ is highly variable, these monovalent vaccines showed efficacy only for certain type of $N$. meningitides. It has proven to be a major challenge to find a product that has a broader coverage of the different strains of serogroup B meningococci. (Bai, Findlow et al. 2011)

\section{Broadening coverage}

Peter et al. designed OMV-based vaccines that should provide broader coverage. A bivalent vaccine was obtained by replacing less immunogenetic Class 3 OMPs in a $\mathrm{H} 44 / 76$ strain with high immunogenic Class 1 OMPs, from $N$. meningitidis strain 2996. Interestingly, the resulting OMVs can induce high antibody titers against both 
strains.(Van Der Ley, Poolman et al. 1992) Later on, a hexavalent OMV was reported. This formulation consists of mixed OMVs in 1:1 ratio from two different strains of $N$. meningitidis PL16215 and PL10124, with each strain expressing three different class 1 OMP variants of Por A.(Claassen, Meylis et al. 1996) In order to further broaden the bacterial coverage, a nonavalent OMV was created on the basis of the hexavalent version. OMVs were added from strain HP1416 engineered to express an additional three Por A subtypes, this OMV-based product triggered high serum bactericidal activity (SBA) titers against certain $N$. meningitidis strain in mice model.(Van Den Dobbelsteen, Van Dijken et al. 2004)

\section{Reverse vaccinology}

Reverse vaccinology is a genome-based method to identify potential antigens for vaccine purposes. It can help to shorten the time for vaccine development and is especially helpful when the pathogen can not be easily cultured in vitro.(Rappuoli 2000) When this technique emerged in 2000, it was used to design an efficient meningococci B vaccine. First, the sequence of the genome of virulent $N$. meningitidis strains was determined. Open reading frames, potentially encoding surface-exposed proteins were identified. A total of 350 candidates were identified, amplified and cloned in E. coli and tested in mice.(Pizza, Scarlato et al. 2000) Five proteins which were able to induce protective immunity and selected for the final multicomponent vaccine. This ' $5 \mathrm{CVMB}^{\prime}$ ' design included three antigens with broad coverage: Neisseria heparin binding antigen (Nhba), fHbp and Neisseria adhesin A ( NadA), and two with less broad coverage the genomic Neisseria antigen (GNA) 2091 and 1030.(Pizza, Scarlato et al. 2000, Giuliani, Adu-Bobie et al. 2006) This formulation appeared effective against $78 \%$ of a representive population of 85 pathogenic meningococcal strains, indicating much broader coverage compared with MenBvac ${ }^{\circledR}$ and MeNZB ${ }^{\circledR}$. (Giuliani, Adu-Bobie et al. 2006) To facilitate large-scale manufacture, four of the five antigens were ultimately expressed as fusion protein, and renamed as '4CMenB', tradename 'Bexsero' (Figure 1).(Mameli, Galli et al. 2015)

The first clinical study showed that $4 C$ MenB induced a higher human serum bactericidal activity than 5 CVMB immunization in adults with a good safety profile. (Mameli, Galli et al. 2015) In Phase II and Phase III studies, infants and children were included, demonstrating a preferred schedule of two doses administered 1 to 6 months apart.(Santolaya, O'Ryan et al. 2012) A follow-up study showed that 77 $94 \%$ of the subjects still had protective antibody titers for up to $18-24$ months after the last vaccination.(Santolaya, O'Ryan et al. 2013) Based on these promising results, $4 C M e n B$ has been integrated into the vaccination program in different countries since 2013. 


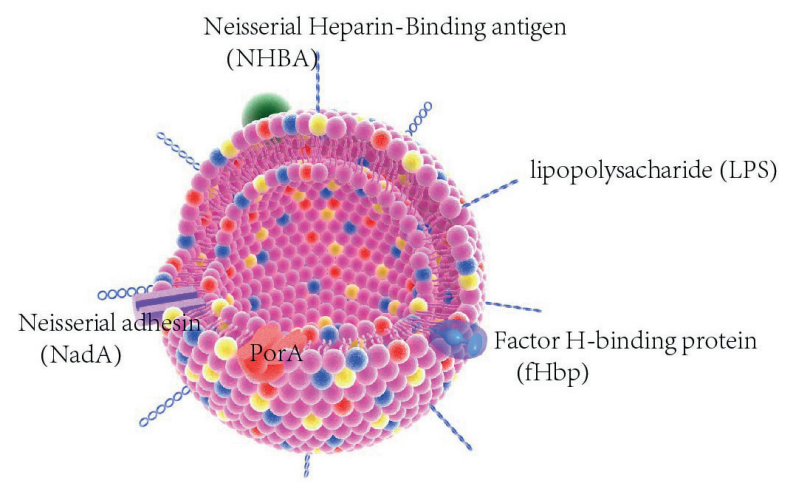

FIGURE 1. Structure of the $4 C M e n B$ OMV vaccine.

\section{Recent developments}

In October 2014 a bivalent recombinant lipidated meningococcal complement factor $\mathrm{H}$ binding protein ( $\mathrm{fHbp}$ ) vaccine (trade name Trumenba ${ }^{\circledR}$ ) was approved by the FDA for patients aged 10 to 25 years. Although $\mathrm{fHbp}$ has a high variability between strains, it can be broadly divided into two subgroups. The vaccine consists of $\mathrm{fHbp}$ of both subgroups. The first clinical studies showed that the vaccine induced bactericidal antibodies against various $N$. meningitides isolates.(Feavers and Maiden 2017)

Dowling et al. attenuated toxicity of $N$. meningitides OMVs by knocking down the gene IpxL1 causing pentacylated LPS to be formed. The OMVs caused a reduced inflammatory response in human leukocytes in vitro while still inducing antigenspecific antibodies in mice. (Dowling, Sanders et al. 2016)

Zhang et al. tried to increase the fHbp content in the OMVs by inserting $\mathrm{fHbp}$ gene into the porB locus, resulting in OMVs without Por B. Interestingly, higher PorA expression was observed. Infant rhesus macaques vaccinated with these OMVs showed antigen-specific antibody responses against the genetically added fHbp. (Zhang, Wen et al. 2016)

\section{Towards heterologous MV platforms}

An example of a novel platform for vaccine development is based on OMVs from an engineered Salmonella enterica serovar Typhimurium strain. A particular advantage of this platform is the fact that the surface of OMVs can be decorated with multiple antigens of choice at a high density. The OMVs are produced by Salmonella enterica serovar Typhimurium strain SL3261, which carries a mutated LPS through msbB 
gene knock-out. In addition, $\triangle$ TolRA was created leading to hypervesiculation of the bacteria.

Antigen loading of the OMVs is achieved using $\mathrm{Hbp}$. As mentioned before, $\mathrm{Hbp}$ is a virulence factor, which is secreted by Gram-negative bacteria via the autotransporter pathway.(Jong, Ten Hagen-Jongman et al. 2007) Autotransporters have three domains, one $\mathrm{N}$-terminal signal peptide, a passenger domain, and a C-terminal -helical domain. the $\mathrm{N}$-terminal signal peptide facilitates crossing of the inner membrane. Then the $\mathrm{C}$-terminal $\beta$-domain helps to target the passenger domain to the outer membrane.(Leo, Grin et al. 2012)

For antigen display, a passenger subdomain was replaced by an ESATs antigen which is secreted by Mycobacterium tuberculosis. After fusing the antigen with Hbp, the cleavage site between the passenger domain and the $\beta$-domain was disrupted, which helps the antigen to remain attached to the surface of the bacteria or OMVs.(Jong, Soprova et al. 2012) Figure 2.

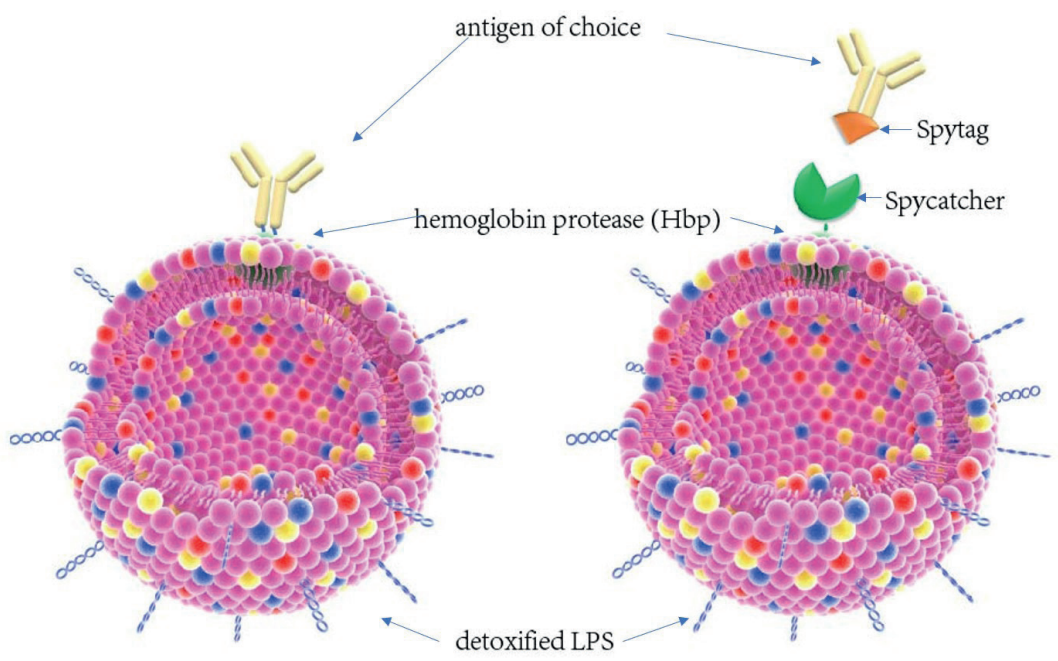

FIGURE 2. Hbp based display of antigens on the surface of OMVs, via fusion with Hbp or equipping $\mathrm{Hbp}$ with a Spytag functionality with cognate Spycatcher coupling of the antigen of choice.

As a result, OMVs from attenuated $S$. Typhimurium was successfully decorated with one, two or three antigens from M. tuberculosis (ESAT6, Ag85B and Rv2660c) and major OMP epitopes from Chlamydia trachomatis.(Daleke-Schermerhorn, Felix et al. 2014) In vitro data showed that the antigen Ag85B delivered by OMVs is able to be recognized and processed by dendritic cells, and subsequently activate $M$. tuberculosis-specific T cells.(Daleke-Schermerhorn, Felix et al. 2014) In addition, when OMVs from the Salmonellae were decorated with high-density Streptococcus pneumoniae antigens pneumococcal surface protein $A$ variants (PspA) and 


\section{Chapter 4}

pneumolysin (Ply) on the surface utilizing this Hbp platform, mice were protected from pneumococcal colonization after intranasal administration.(Kuipers, DalekeSchermerhorn et al. 2015) When compared with previously mentioned membrane/ transmembrane fusion methods, the Hbp platform allows higher density display of antigens. An additional advantage is the fact that it is better suited for antigens with larger sizes and complicated structures.(Jong, Soprova et al. 2012) This Hbpbased platform was further explored to developed OMVs against by decorating with fragments of protective protein.(Kuipers, Daleke-Schermerhorn et al. 2015) One of the disadvantages of the $\mathrm{Hbp}$ platform is that the expression of the $\mathrm{Hbp}$ chimera might be lower when the number, size, and complexity of the coupled antigens increases.(Houben, de Jonge et al. 2018)

One of the solutions to circumvent these issues is to use the Hbp platform together with protein ligation technologies such as 'SpyTag-SpyCatcher' or 'SnoopTagSnoopCatcher'. 'SpyTag' is a short peptide that can form a covalent bond when it encounters its protein partner 'SpyCatcher'.(Reddington and Howarth 2015) Due to the strong binding, it can serve as 'superglue' to fuse antigens of interest to OMVs. Bart et al decorated the MV platform with pneumococcal antigens PspA $\alpha$ and SP1690 by firstly coupling the SpyTag to Hbps, and subsequently fusing the purified pneumococcal antigens to Spycatcher.(Houben, de Jonge et al. 2018)

\section{Final remarks}

MVs from Gram-positive and Gram-negative bacteria are attractive vaccine platforms. Genetic engineering approaches enable precise tailoring of MVs to achieve safe, highly immunogenic and efficient vaccines. The first OMV-based engineered vaccine provides an example for the rational design of such platforms. Currently, new opportunities are emerging with heterologous engineered OMV vaccines that can have broad therapeutic activity by displaying various antigens on their surface.

\section{Acknowledgement}

Prof. Rob Willems (Department of Medical Microbiology, UMC Utrecht) is acknowledged for giving suggestions for this manuscript. Prof. Joen Luirink (Faculty of Science, Molecular Microbiology, Vrije Universiteit Amsterdam) is acknowledged for discussion on the work he has published. 


\section{Bacterial membrane vesicles as promising vaccine candidates}

\section{References}

https://www.uniprot.org/uniprot/A0Q4N6.

http://aberabio.com/technology/.

Bai, X., et al. (2011). "Recombinant protein meningococcal serogroup B vaccine combined with outer membrane vesicles." 11(7): 969-985.

Baker, J. L., et al. (2014). "Microbial biosynthesis of designer outer membrane vesicles." Current opinion in biotechnology 29: 76-84.

Beveridge, T. J. (1999). "Structures of gram-negative cell walls and their derived membrane vesicles." Journal of bacteriology 181(16): 4725-4733.

Bjune, G., et al. (1991). "Effect of outer membrane vesicle vaccine against group B meningococcal disease in Norway." The Lancet 338(8775): 1093-1096.

Caron, F., et al. (2012). "Evolution of immune response against Neisseria meningitidis B: 14: P1. 7, 16 before and after the outer membrane vesicle vaccine MenBvac." 30(34): 5059-5062.

Caron, F., et al. (2011). "From tailor-made to ready-to-wear meningococcal B vaccines: longitudinal study of a clonal meningococcal B outbreak." 11(6): 455-463.

Chatterjee, S. and J. Das (1966). "Secretory activity of Vibrio cholerae as evidenced by electron microscopy." Electron Microscopy: 139-140.

Chen, D. J., et al. (2010). "Delivery of foreign antigens by engineered outer membrane vesicle vaccines." Proceedings of the National Academy of Sciences 107(7): 3099-3104.

Chen, L., et al. (2016). "Outer membrane vesicles displaying engineered glycotopes elicit protective antibodies." Proceedings of the National Academy of Sciences 113(26): E3609-E3618.

Choi, J.-W., et al. (2017). "Tiny RNAs and their voyage via extracellular vesicles: secretion of bacterial small RNA and eukaryotic microRNA." Experimental Biology and Medicine 242(15): 1475-1481.

Claassen, I., et al. (1996). "Production, characterization and control of a Neisseria meningitidis hexavalent class 1 outer membrane protein containing vesicle vaccine." 14(10): 1001-1008.

Daleke-Schermerhorn, M. H., et al. (2014). "Decoration of outer membrane vesicles with multiple antigens by using an autotransporter approach." Appl. Environ. Microbiol. 80(18): 5854-5865.

Daleke-Schermerhorn, M. H., et al. (2014). "Decoration of outer membrane vesicles with multiple antigens by using an autotransporter approach." $\mathbf{8 0}(18)$ : 5854-5865.

Deatherage, B. L., et al. (2009). "Biogenesis of bacterial membrane vesicles." Molecular microbiology 72(6): 1395-1407.

Dorward, D. W. and C. F. Garon (1990). "DNA is packaged within membrane-derived vesicles of Gramnegative but not Gram-positive bacteria." Appl. Environ. Microbiol. 56(6): 1960-1962.

Dowling, D. J., et al. (2016). "A meningococcal outer membrane vesicle vaccine incorporating genetically attenuated endotoxin dissociates inflammation from immunogenicity." 7: 562.

Fantappie, L., et al. (2014). "Antibody-mediated immunity induced by engineered Escherichia coli OMVs carrying heterologous antigens in their lumen." Journal of extracellular vesicles 3(1): 24015.

Feavers, I. M. and M. C. J. C. V. I. Maiden (2017). "Recent progress in the prevention of serogroup B meningococcal disease." 24(5): e00566-00516.

Gerritzen, M. J., et al. (2017). "Bioengineering bacterial outer membrane vesicles as vaccine platform." 35(5): 565-574.

Giuliani, M. M., et al. (2006). "A universal vaccine for serogroup B meningococcus." Proceedings of the National Academy of Sciences 103(29): 10834-10839.

Gnopo, Y. M., et al. (2017). "Designer outer membrane vesicles as immunomodulatory systemsReprogramming bacteria for vaccine delivery." Advanced drug delivery reviews 114: 132-142.

Gujrati, V., et al. (2014). "Bioengineered bacterial outer membrane vesicles as cell-specific drug-delivery vehicles for cancer therapy." 8(2): 1525-1537.

Haurat, M. F., et al. (2011). "Selective sorting of cargo proteins into bacterial membrane vesicles." Journal of biological chemistry 286(2): 1269-1276. 


\section{Chapter 4}

Høiby, E., et al. (1990). "Serological responses in Norwegian adult volunteers to a meningococcal 15: P1. 16 outer membrane vesicle vaccine (phase II studies)." 241-246.

Holst, J., et al. (2005). "The concept of "tailor-made", protein-based, outer membrane vesicle vaccines against meningococcal disease." 23(17-18): 2202-2205.

Houben, D., et al. (2018). "Display of recombinant proteins on bacterial outer membrane vesicles by using protein ligation." Appl. Environ. Microbiol. 84(8): e02567-02517.

Jong, W. S., et al. (2012). "A structurally informed autotransporter platform for efficient heterologous protein secretion and display." Microbial cell factories 11(1): 85.

Jong, W. S., et al. (2012). "A structurally informed autotransporter platform for efficient heterologous protein secretion and display." 11(1): 85.

Jong, W. S., et al. (2007). "Limited tolerance towards folded elements during secretion of the autotransporter Hbp." 63(5): 1524-1536.

Kaaijk, P., et al. (2013). "Preclinical safety and immunogenicity evaluation of a nonavalent PorA native outer membrane vesicle vaccine against serogroup B meningococcal disease." 31(7): 1065-1071.

Kim, J.-Y., et al. (2008). "Engineered bacterial outer membrane vesicles with enhanced functionality." Journal of molecular biology 380(1): 51-66.

Kim, S.-H., et al. (2009). "Structural modifications of outer membrane vesicles to refine them as vaccine delivery vehicles." Biochimica et Biophysica Acta (BBA)-Biomembranes 1788(10): 2150-2159.

Klimentová, J. and J. Stulík (2015). "Methods of isolation and purification of outer membrane vesicles from gram-negative bacteria." Microbiological research 170: 1-9.

Konings, W., et al. (1973). "New procedure for the isolation of membrane vesicles of Bacillus subtilis and an electron microscopy study of their ultrastructure." Journal of bacteriology 116(3): 1456-1465.

Kuipers, K., et al. (2015). "Salmonella outer membrane vesicles displaying high densities of pneumococcal antigen at the surface offer protection against colonization." Vaccine 33(17): 2022-2029.

Kuipers, K., et al. (2015). "Salmonella outer membrane vesicles displaying high densities of pneumococcal antigen at the surface offer protection against colonization." 33(17): 2022-2029.

Kulp, A. and M. J. Kuehn (2010). "Biological functions and biogenesis of secreted bacterial outer membrane vesicles." Annual review of microbiology 64: 163-184.

Lee, D. H., et al. (2011). "Adjuvant effect of bacterial outer membrane vesicles with penta-acylated lipopolysaccharide on antigen-specific T cell priming." Vaccine 29(46): 8293-8301.

Lee, E. Y., et al. (2009). "Gram-positive bacteria produce membrane vesicles: proteomics-based characterization of Staphylococcus aureus-derived membrane vesicles." Proteomics 9(24): 54255436.

Lee, S.-R., et al. (2009). "Multi-immunogenic outer membrane vesicles derived from an MsbB-deficient Salmonella enterica serovar typhimurium mutant." J Microbiol Biotechnol 19(10): 1271-1279.

Lee, T.-Y., et al. (2017). "Outer membrane vesicles harboring modified lipid A moiety augment the efficacy of an influenza vaccine exhibiting reduced endotoxicity in a mouse model." Vaccine 35(4): 586-595.

Leo, J. C., et al. (2012). "Type V secretion: mechanism (s) of autotransport through the bacterial outer membrane." 367(1592): 1088-1101.

Lötvall, J., et al. (2014). Minimal experimental requirements for definition of extracellular vesicles and their functions: a position statement from the International Society for Extracellular Vesicles, Taylor \& Francis.

Mamat, U., et al. (2015). "Detoxifying Escherichia coli for endotoxin-free production of recombinant proteins." Microbial cell factories 14(1): 57.

Mameli, C., et al. (2015). "The multicomponent meningococcal serogroup B vaccine (4CMenB): origin, composition, health impact and unknown aspects." Future microbiology 10(10): 1579-1598.

Mameli, C., et al. (2015). "The multicomponent meningococcal serogroup B vaccine (4CMenB): origin, composition, health impact and unknown aspects." 10(10): 1579-1598.

McBroom, A. J., et al. (2006). "Outer membrane vesicle production by Escherichia coli is independent of membrane instability." Journal of bacteriology 188(15): 5385-5392. 
McBroom, A. J. and M. J. Kuehn (2007). "Release of outer membrane vesicles by Gram-negative bacteria is a novel envelope stress response." Molecular microbiology 63(2): 545-558.

Mitra, S., et al. (2016). "Development of a cost-effective vaccine candidate with outer membrane vesicles of a tolA-disrupted Shigella boydii strain." Vaccine 34(15): 1839-1846.

Oster, P., et al. (2005). "MeNZB ${ }^{\mathrm{TM}}$ : a safe and highly immunogenic tailor-made vaccine against the New Zealand Neisseria meningitidis serogroup B disease epidemic strain." Vaccine 23(17-18): 2191-2196.

Pérez, A. R. and F. J. R. c. d. m. t. Meneses (1999). "VA-Mengoc-BC vaccine: its impact on meningococcal disease in children 1-4 years old." 51(3): 189-193.

Pizza, M., et al. (2000). "Identification of vaccine candidates against serogroup B meningococcus by wholegenome sequencing." Science 287(5459): 1816-1820.

Ranallo, R. T., et al. (2010). "Virulence, inflammatory potential, and adaptive immunity induced by Shigella flexneri msbB mutants." 78(1): 400-412.

Rappuoli, R. (2000). "Reverse vaccinology." Current opinion in microbiology 3(5): 445-450.

Reddington, S. C. and M. J. C. o. i. c. b. Howarth (2015). "Secrets of a covalent interaction for biomaterials and biotechnology: SpyTag and SpyCatcher." 29: 94-99.

Santolaya, M. E., et al. (2013). "Persistence of antibodies in adolescents 18- 24 months after immunization with one, two, or three doses of 4 CMenB meningococcal serogroup B vaccine." 9(11): 2304-2310.

Santolaya, M. E., et al. (2012). "Immunogenicity and tolerability of a multicomponent meningococcal serogroup $B(4 C M e n B)$ vaccine in healthy adolescents in Chile: a phase $2 b / 3$ randomised, observerblind, placebo-controlled study." 379(9816): 617-624.

Sierra, G., et al. (1991). "Vaccine against group B Neisseria meningitidis: protection trial and mass vaccination results in Cuba." NIPH annals 14(2): 195-207; discussion 208-110.

Toyofuku, M., et al. (2018). "Types and origins of bacterial membrane vesicles." Nature Reviews Microbiology: 1.

Traber, K. E., et al. (2008). "agr function in clinical Staphylococcus aureus isolates." 154(Pt 8): 2265.

Turnbull, L., et al. (2016). "Explosive cell lysis as a mechanism for the biogenesis of bacterial membrane vesicles and biofilms." Nature communications 7: 11220.

Van de Waterbeemd, B., et al. (2010). "Improved OMV vaccine against Neisseria meningitidis using genetically engineered strains and a detergent-free purification process." Vaccine 28(30): 4810-4816.

Van Den Dobbelsteen, G., et al. (2004). From HexaMen to NonaMen: expanding a multivalent PorAbased meningococcal outer membrane vesicle vaccine. 14th International Pathogenic Neisseria Conference. Milwaukee WI, Sep.

Van Der Ley, P., et al. (1992). "Construction of a multivalent meningococcal vaccine strain based on the class 1 outer membrane protein." 60(8): 3156-3161.

Van Der Ley, P., et al. (2001). "Modification of Lipid A Biosynthesis inNeisseria meningitidis IpxL Mutants: Influence on Lipopolysaccharide Structure, Toxicity, and Adjuvant Activity." 69(10): 5981-5990.

van der Pol, L., et al. (2015). "Outer membrane vesicles as platform vaccine technology." Biotechnology journal 10(11): 1689-1706.

Wagner, T., et al. (2018). "Enterococcus faecium produces membrane vesicles containing virulence factors and antimicrobial resistance related proteins." Journal of proteomics 187: 28-38.

Wang, X., et al. (2018). "Release of Staphylococcus aureus extracellular vesicles and their application as a vaccine platform." Nature communications 9(1): 1379.

Watkins, H. C., et al. (2017). "Safe recombinant outer membrane vesicles that display M2e elicit heterologous influenza protection." Molecular Therapy 25(4): 989-1002.

Zariri, A., et al. (2016). "Meningococcal outer membrane vesicle composition-dependent activation of the innate immune response." 84(10): 3024-3033.

Zhang, L., et al. (2016). "Improving the immunogenicity of a trivalent Neisseria meningitidis native outer membrane vesicle vaccine by genetic modification." 34(35): 4250-4256.

Zhang, Q., et al. (2015). "Commensal bacteria direct selective cargo sorting to promote symbiosis." Nature immunology 16(9): 918. 


\section{Chapter 5}

\section{Membrane Vesicles as nano-weapons for combating $E$. faecium infection}

Linglei Jiang ${ }^{1}$, Fernanda Paganelli², Janetta Top ${ }^{2}$, Marcel Fens ${ }^{4}$, Najrana Mustafia', Kok van Kessel ${ }^{2}$, Robin Hartman ${ }^{5}$, Pieter Vader ${ }^{1.5}$, Mona Johannessen ${ }^{3}$, Wim E. Hennink ${ }^{4}$, Rob Willems ${ }^{2}$ and Raymond M. Schiffelers ${ }^{1 *}$

1. CDL Research, Division LAB, Faculty of Medicine, University Medical Center Utrecht, Utrecht University, Utrecht, the Netherlands

2. Department of Medical Microbiology, UMC Utrecht, the Netherlands

3. Research Group of Host-Microbe Interactions, Department of Medical Biology, Faculty of Health Sciences, UiT-The Arctic University of Norway, Norway

4. Utrecht Institute for Pharmaceutical Sciences (UIPS), Utrecht University, the Netherlands

5. Department of Cardiology, UMC Utrecht, the Netherlands

Corresponding author: R.Schiffelers@umcutrecht.nl 


\section{Chapter 5}

\section{Abstract}

Membranevesicles (MVs) released by bacteria have important roles in bacteria-bacteria and bacteria-host communication. Research focused on MVs from Gram-positive bacteria is increasing in recent years, after largely being ignored in the period before that. In this study, we investigated the morphology, size, yield, protein compositions and immunogenicity of MVs released from the nosocomial pathogen Gram-positive bacterium Enterococcus faecium (E. faecium) wild-type (WT) strain E1162 and two E1162 isogenic mutants yajC and AltA. Populations with different morphologies were observed among MVs from E. faecium. Qualitative and quantitative proteomics of MVs revealed that 247, 314 and 325 proteins were enriched in MVs from WT, $\Delta y a j C$ and $\triangle A l t A$, respectively when compared to the predicted total number of proteins $(>2000)$ of E1162. This enrichment indicates that there is a selective protein sorting mechanism during MV biogenesis. Furthermore, it was shown that both E1162 WT MV and E1162 $\triangle$ yajC MV contained a higher abundance of potential immunogenic proteins such as Pbp5, LysM, AdcAfm 2410 and AdcAfm 2344 when compared with E1162 $\Delta$ AltA. Finally, it was shown that both E1162 MVs and $\Delta y a j C$ MVs can illicit cytokine release and long-term immune response in a murine model, which suggests that $E$. faecium-derived MVs are promising vaccine candidates against E. faecium infection. 


\section{Introduction}

Enterococcus faecium is a Gram-positive bacterium. They can be commensal bacteria in the gastrointestinal tract of insects, animals and humans. However, $E$. faecium emerged as an important nosocomial pathogen causing infections such as bacteremia, neonatal meningitis, endocarditis and urinary tract infections during the last three decades. (Heikens, Bonten et al. 2007) One of the possible reasons for this emergence is the fact that $E$. faecium is intrinsically resistant to antibiotics, but also that this bacterium is able to efficiently and frequently acquire novel resistance traits. This multi-resistance nature hampers efficient treatment of $E$. faecium infections necessitating the development of a new anti- E. faecium approaches.

One attractive approach may lie in the field of vesicles. Both eukaryotes and prokaryotes constitutively release nano-sized phospholipid bi-layered membranebound structures. Those structures are also referred to as 'extracellular vesicles (EVs)'. They play important roles in cell-cell communication by transferring cargoes such as proteins and nucleic acids, thereby influencing the phenotype of the recipient cells. (Yáñez-Mó, Siljander et al. 2015) EVs shed by bacteria are also termed 'membrane vesicles' (MVs). Evidence shows that both Gram-negative bacteria and Gram-positive bacteria can release MVs. (Brown, Wolf et al. 2015) When bacterial MVs interact with immune cells, they may be able to elicit an immune response which makes bacterial MVs prospective vaccination candidates.(Collins 2011)

The biogenesis of MVs for Gram-negative bacteria is better understood compared with Gram-positive bacteria. Until recently, three models have been proposed for the biogenesis of MVs from Gram-negative bacteria, also referred as 'outer membrane vesicles'. The first model suggests that the accumulation of the peptidoglycan fragments or misfolded proteins in the periplasmic space could exert a turgor pressure to facilitate the pinch off of the outer membrane (OM). A second model suggests that if the growth rate of $\mathrm{OM}$ is higher than the underlying peptidoglycan, it will result in loss of interaction between OM and PGL which allows the vesiculation of OM. The third model suggests that negatively charged B-band lipopolysaccharide (LPS) can be selectively packed in the vesicles, and the abundance of B-band LPS in certain regions of the membrane result in the bending of OM due to charge repulsion. (Kulp and Kuehn 2010, Haurat, Elhenawy et al. 2015).

Recently, another new OMV biogenesis mechanism was proposed, which suggests that deletion of a phospholipid transporter $\mathrm{Vac} / \mathrm{Yrb}$ ATP-binding cassette can facilitate phospholipid ( $P L$ ) accumulation in the $\mathrm{OM}$. As $\mathrm{PL}$ is known for maintaining the asymmetry in $\mathrm{OM}$, the accumulation of PL thus initiates the outward budding of OM. As a result, more OMV shed off the outer membrane. (Roier, Zingl et al. 2016) 


\section{Chapter 5}

In contrast to Gram-negative bacteria, less is known about how MVs escape the thick cell wall in Gram-positive bacteria. One hypothesis for the MVs biogenesis from Gram-positive bacteria is that endolysin or other peptidoglycan-damaging enzymes/ can trigger the 'bubbling cell death' of Gram-positive bacteria, and the bubbling further leads to more production of MVs. (Toyofuku, Nomura et al. 2019)

A previous study by Wagner et. al. has shown that four clinically relevant $E$. faecium strains can produce $M V s$, and their protein cargoes are strain and culture condition dependent. Several vaccine candidates were identified on the MVs. (Wagner, Joshi et al. 2018) Another study showed that MVs from E. faecium can induce stronger proinflammatory responses in host cells when E. faecium were cultured with antibiotics. (Kim, Kim et al. 2019)

In this study, we selected $E$. faecium strain E1162, a nosocomial clinical isolate, to assess MV formation. Strain E1162, which is resistant to a variety of antibiotics including ampicillin, was firstly isolated from the blood of a patient in Lyon. (Zhang, Paganelli et al. 2012) In addition, two isogenic mutants of E1162 were included (E1162 yajC and E1162 AtlA), as these mutants expressed phenotype traits of increased protein release, which could be indicative of enhanced $E$. faecium MV biogenesis.

Here we report the isolation and characterization of MVs from E. faecium strain E1162 and its isogenic mutants. Their yield, size and protein profiles were compared. Vaccine candidates from the MVs were identified. and the potential to use these MVs as vaccine candidates were evaluated in a murine model. This study aims to understand the genetic impact on the release, composition and biological function of MVs, and select a MVs-based vaccine candidate against E. faecium infection for clinical translation.

\section{Results}

\section{Culture of bacteria and medium selection}

Bacterial strains used in this studied were summarized in Table 1.

To select the optimal culture medium for E1162 MV production, four media were tested: BHI (Brain Heart Infusion Broth), Tryptic Soy Broth (TSB), M1 and Lysogeny Broth (LB). The growth rates of E1162 growth rate in the four different culture media is shown in Figure S1. The growth rate follows the order BHI $>T S B>M 1>L B$ medium. The slow growth rate of E1162 in LB medium. disqualified this broth. Subsequently, the three remaining media were evaluated for the production of MVs by the E1162 strain. The production of MVs in TSB medium was virtually absent as observed by transmission electron microscopy (Figure S2). In BHI medium, apparently, a much 
higher MV yield was obtained. However, the vesicles isolated here were for a large part already present in the medium without culturing of the bacteria (Figure S3). The animal source material for this broth is likely responsible for this vesicular contamination. As a result of this high background $\mathrm{BHI}$ medium was also discarded. This resulted in the selection of the M1 medium as E. faecium showed a reasonable growth rate in this medium and MV production in a clean background.

TABLE 1. Information on E. faecium strains and the isogenic mutants studied

\begin{tabular}{lll}
\hline Strain or plasmids & \multicolumn{1}{c}{ Relevant characteristics } & \multicolumn{1}{c}{ reference } \\
\hline E1162 (WT) & $\begin{array}{l}\text { an ampicillin-resistant clinical isolate from a } \\
\text { bloodstream infection }\end{array}$ & (Zhang, Paganelli et al. 2012) \\
\hline yajC & $\begin{array}{l}\text { Deletion of yajC transmembrane gene. which is } \\
\text { involved in stabilization of protein in the bacterial } \\
\text { surface. }\end{array}$ & (Paganelli unpublished data) \\
\hline AtlA & $\begin{array}{l}\text { Deletion of the major autolysin gene, which is } \\
\text { an endogenous lytic enzyme that hydrolyzes the } \\
\text { peptidoglycan components of cells }\end{array}$ & (Paganelli, Willems et al. 2013) \\
&
\end{tabular}

\section{MVs isolation and characterization}

TEM imaging of Optiprep fractions of E. faecium E1162 WT. yajC and AltA mutants revealed that fractions containing 15-30\% lodixanol contains the majority of MVs showing a classical cup-shaped morphology (Figure S4). Hence, these fractions were pooled for subsequent studies. Interestingly, different morphological subtypes of MVs were observed, which included double membrane vesicles, double vesicles, pleomorphic vesicles, electron-dense vesicles and filamentous vesicles/filaments (Figure 1). These results provide evidence that subpopulations can be distinguished for EVs from prokaryotes similar to EVs of eukaryotes (Lässer, Jang et al. 2018). For yajC and AltA mutants similar subpopulation phenomena were observed. (Figure S5) Cryo-EM data revealed the sphere-shaped morphology of MVs from all three samples, and the size of majority of MVs are within 20-60 nm (Figure 2).

Subsequently, the MVs were further characterized by analyzing the protein composition of MVs of E1162 WT and isogenic mutants using by SDS-PAGE and silverstaining and size measurements. SDS-PAGE analysis indicated several differences in protein profiles of the tested strains. For example, the protein with molecular weight of $130 \mathrm{kDa}$ was uniquely present in the yajC mutant but not in WT and AltA (Figure 3a). The size of MVs from WT and its mutants was measured by nanoparticle tracking analysis (NTA), broad size distribution was observed of these three MVs (data not shown) and their mode size has no significant differences (Figure S6). And this result is in agreement with the observation by TEM results (Figure 1). Particle numbers were counted for each MV samples, and it was found out that the MVs production from 


\section{Chapter 5}

E1162 yajC was approximately 3-fold higher than that of E1162 WT and AltA, the latter two exhibited a similar particle concentration (Figure 3b).
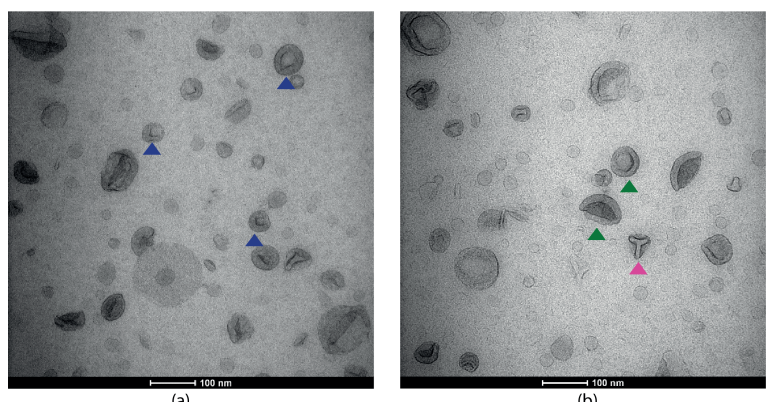

double membrane vesicles

A double vesicles

- pleomorphic vesicles

$\Delta$ electron-dense vesicles

filamentous vesicles

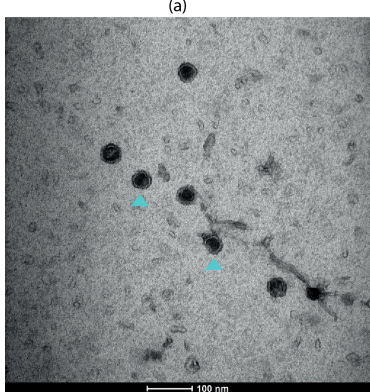

(c)

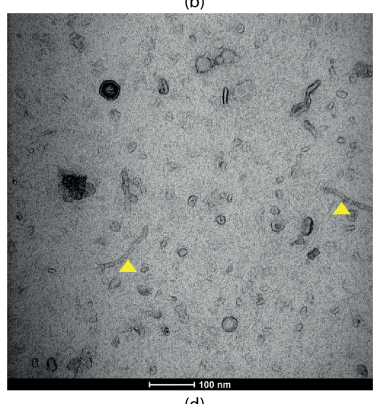

FIGURE 1. MVs from WT strain were purified by density gradient ultracentrifugation (Optiprep). and vesicles present in different fractions were visualized by TEM. Vesicles subpopulations were observed.

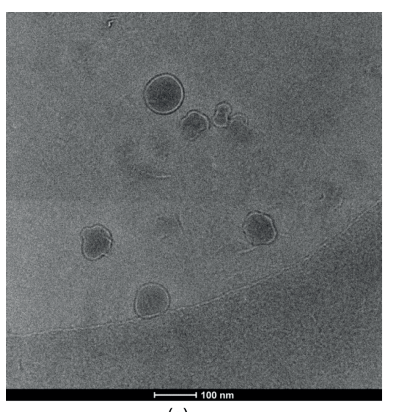

(a)

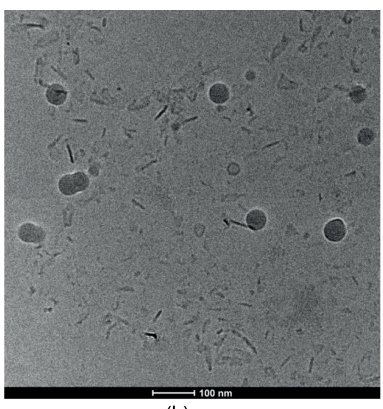

(b)

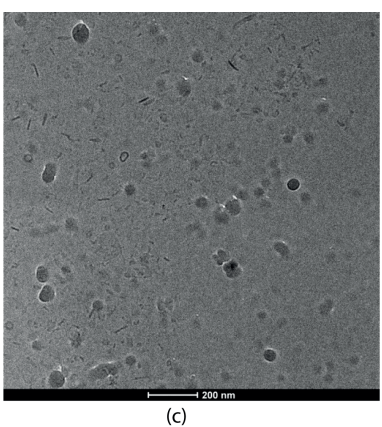

(c)

FIGURE 2. Cryo-EM images of MVs from WT (a), yajC mutant (b) and AltA mutant (c) 


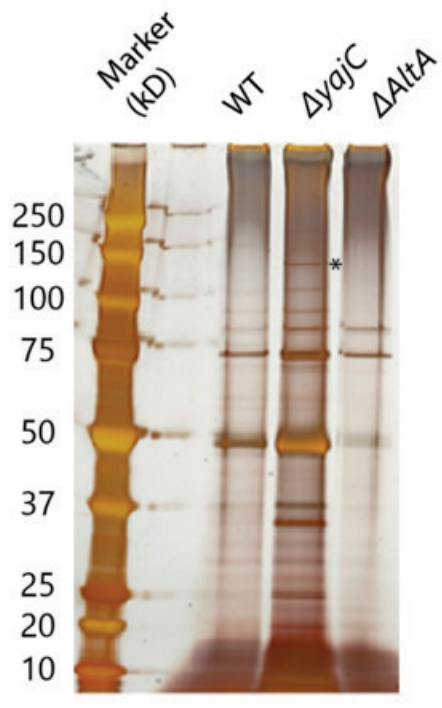

(a)

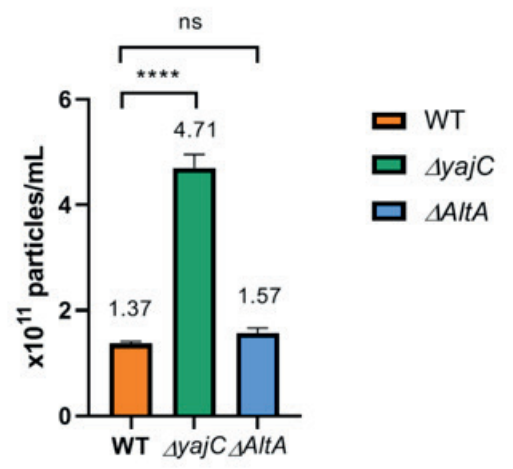

(b)

FIGURE 3. Protein compositions. size and particle concentration of MVs from E1162 WT and its isogenic mutants. Silver staining after SDS-PAGE showing the protein profiles of MVs from WT and its isogenic mutants, $130 \mathrm{kDa}$ band uniquely present by yajC is indicated by asterisk (a); particle concentration of MVs from WT and its mutants measured by NTA (standard error $\left.=4.3 \times 10^{7}, n=3\right)(b)$.

\section{Qualitative and quantitative proteomics}

To explore the protein profiles in more depth. both qualitative and quantitative proteomics analysis were performed for the three MVs samples. In total, 247, 314 and 325 different proteins were identified in MVs from WT, yajC and AltA respectively, which is approximately $10 \%$ of the predicted number of 2696 proteins encoded by E1162. (https://www.uniprot.org/proteomes/UP000004426) This suggests that there is a selective protein sorting process MV biogenesis. 120 proteins are commonly identified in all three strains of MVs. A closer inspection of the proteins unique present in each MV sample, those proteins are predicted to be involved in translation, ribosomal structure and biogenesis were recovered in WT MVs, while proteins related to amino acids/carbohydrate transport and metabolism were found in yajC MV and $\triangle$ AltA MV (Figure 4a).

To quantify the abundance of proteins in all three MV samples, a label-free quantitative proteomic approach was used. The principal component analysis (PCA) plot shows a strong clustering of triplicates for each of the MV types (Figure 4b). Interestingly, muramidase-2 which is a peptidoglycan hydrolase accounts for a high percentage of recovered peptides in all three MV sample, with 33.3\% in WT MV, and $61.8 \%$ and $50.7 \%$ in its yajC and AltA mutant, respectively (Table S1). while peptides 


\section{Chapter 5}

belonging to muramidase-2 only accounts for 3-18\% of total peptide content of E1162 under different culture condition. (Sinnige, de Been et al. 2015)

Next, we analyzed the presence of surface exposed proteins in these MVs as we anticipated that these proteins could potentially elicit a protective immune response. Amongst the surface-exposed proteins, a peptidoglycan-binding protein LysM (LysM), D-alanyl-D-alanine carboxypeptidase (DdcP), low affinity penicillin-binding protein 5 (PBP5) and the peptidyl-prolyl cis-trans isomerase (PpiC) were identified. (Romero-Saavedra, Laverde et al. 2014). In addition, the major secreted protein SagA (Kropec, Sava et al. 2011) and metal-binding proteins. such as the manganese ABC transporter substrate-binding lipoprotein (PsaAfm) and the zinc $A B C$ transporter substrate-binding lipoprotein (AdcAfm) (Romero-Saavedra, Laverde et al. 2015) recovered in all MV samples are also considered immunogenic proteins and potential vaccine candidates. Quantitative analysis results showed that both WT MV and yajC MV contains higher abundance of immunogenic proteins such as pbp5, LysM, AdcAfm 2410 and AdcAfm 2344 than AltA, which suggests that WT MV and $\triangle y a j C$ MV can potentially be promising vaccine hits (Figure $4 \mathrm{C}$ and Table S2). This, in combination with our earlier observation that higher yields of MVs were obtained from E1162 WT and E1162 yajC decided us to focus for further analysis on MVs from E1162 WT and yajC.

\section{yajC MV illicit short-term and long-term immune response in a murine model}

To investigate the possibility of using MVs as a vaccine platform, boost immunizations with $5 \mathrm{mg}$ MVs (quantify by total MV proteins) was administered to mice by subcutaneous (sc) and intraperitoneal (ip) route at week 0 , week 2 and week 4 respectively (Figure $5 a$ ). Both immunization protocols did not result in obvious adverse effects when monitoring the behavior and body weight of the experimental mice (Figure S7).

To study the cytokine release profile, blood samples were collected $18 \mathrm{~h}$ after injection and analyzed with Olink proximity extension assay (using the mouse plasma proteome panel). As is shown in Figure 5b, both WT and yajC elicited an acute immune response, reflected by increasing cytokine expression levels. For example. proinflammatory cytokine IL6 and IL1 are elevated when WT MVs were injected by the sc route. Anti-inflammatory cytokine IL10, IL1a, IL5 were elevated when yajC MVs was administered via the ip route. Of note, cytokines elevated by yajC MV after ip administration cluster well in STRING database, which suggests that there are a lot of protein-protein interactions within this set of cytokines. The elevated cytokines mainly belong to the functional categories "cytokine activity", "cytokine receptor binding", "signaling receptor binding" and "molecular function regulator". 
(a)

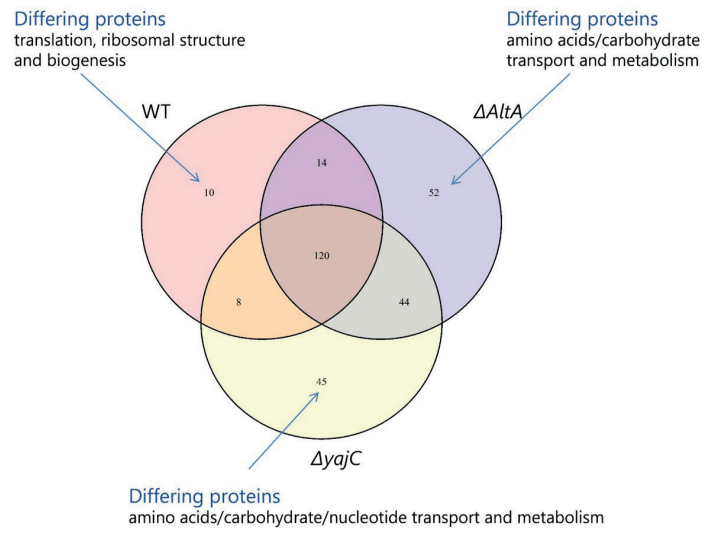

(b)

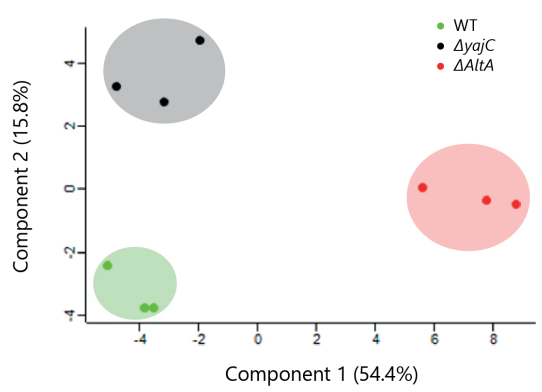

(c)

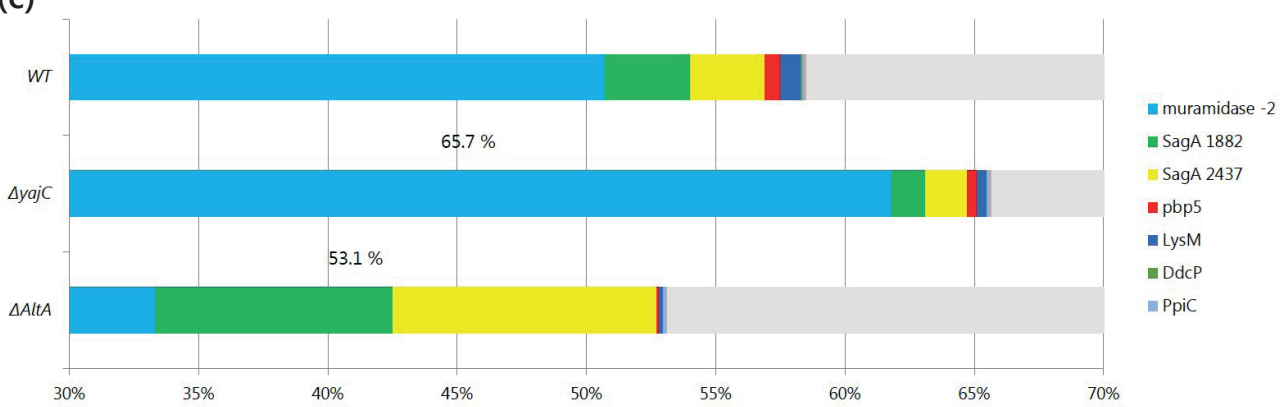

FIGURE 4. Venn diagram of proteins identified in MVs from WT and its isogenic mutants (a); PCA plot of MVs from WT and its isogenic mutants $(n=3)(b)$. quantitative analysis of protein compositions of all three MVs (c).

Figure $5 c$ shows that $E$. faecium binding $\lg G$ levels were significantly increased 4 weeks after ip injection with WT MV and yajC MV and these high IgG levels were maintained at least until 6 weeks after immunization by both sc and ip administration routes. Interestingly, $\triangle y a j C \mathrm{MV}$ induced slightly higher IgG plasma concentrations when injected by same route at week 6 , which suggests that $\triangle y a j C M V$ s have stronger immunogenic properties than WT MVs at the same dose. 
Chapter 5

(a)

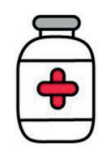

injected MV samples

1. mock MVs

2. WT MVs

3. $\triangle$ Yajc MVs

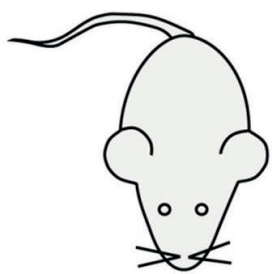

week 4

week 0

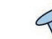

\&
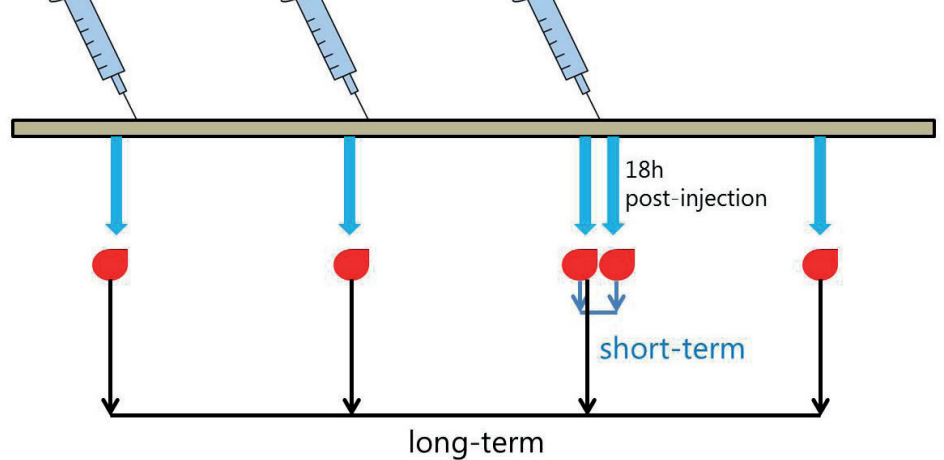

(b)

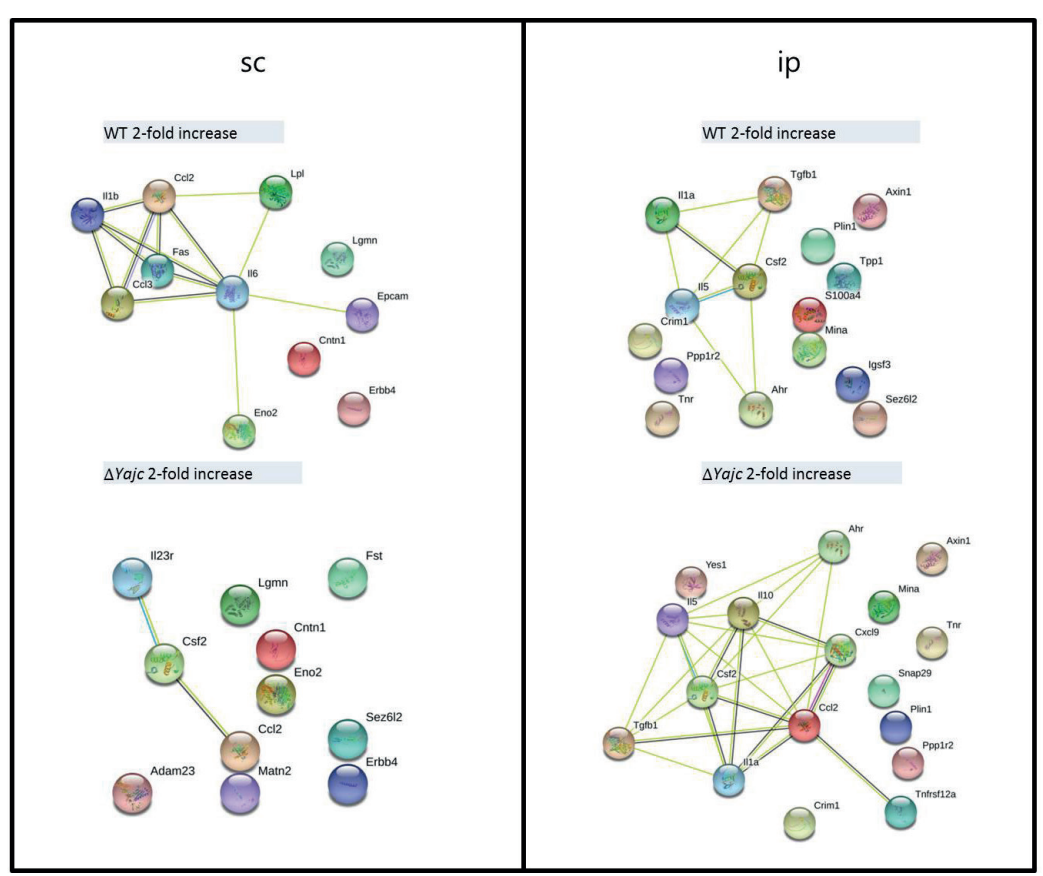


(c)

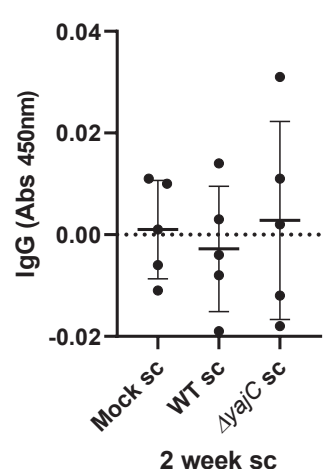

2 week sc

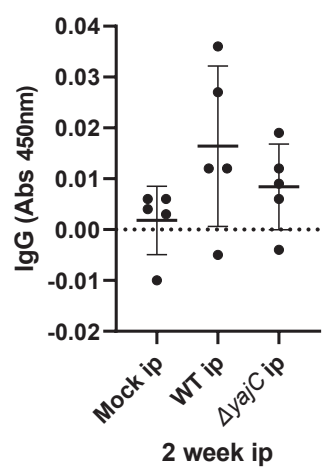

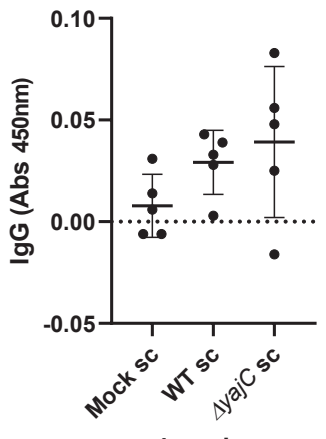

4 week sc

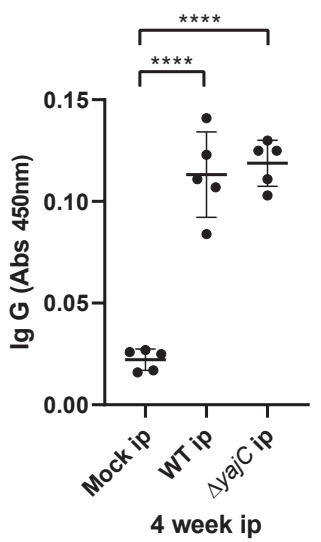

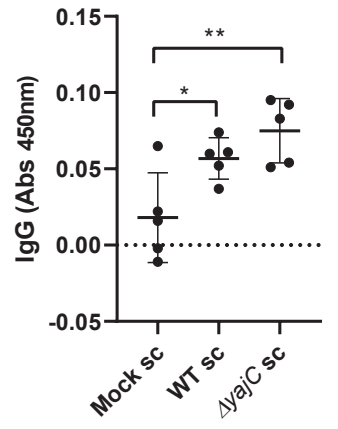

6 week sc

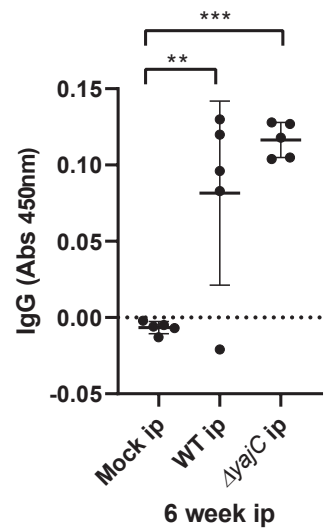

FIGURE 5. Schematic representation of in vivo study in murine model (a); functional protein association networks in STRING database in acute immune response study (b); Anti-E. faecium IgG expression levels measured during the course of the experiment (2, 4 and 6 weeks), plasma samples were diluted 100 times (c).

\section{Discussion \& conclusion}

Nosocomial infections due to multidrug-resistant E. faecium are an increasing problem in hospitals and the multidrug-resistance nature of this pathogen significantly comprise treatment of infected patients. Therefore, alternative treatment and preventive strategies and MVs could represent an attractive new class of vaccine candidates. also for pathogenic Gram-positive bacteria like E. faecium. However, there is limited information on using MVs from Gram-positive bacteria as vaccine platform. In this study, we showed that both E.faecium E1162 WT and its isogenic 


\section{Chapter 5}

mutants $\triangle y a j C$ and $\triangle A l t A$ release $M V s$, and these $M V s$ varies regarding their yield, size and protein profiles. Importantly. we provide the first-in-vivo information on using MVs as vaccine candidates.

When analyzing the production of MV from E1162 and its isogenic mutants E1162yajC and E1162AltA, five morphologically distinct subpopulations of MVs were recovered from each strain providing evidence that MV subpopulations exist in prokaryotes similarly as for eukaryotes where this is well established (Lässer, Jang et al. 2018).

The yield of MVs from E1162 WT and its isogenic mutants yajC was significantly different. In the mutant in which the yajC gene, putatively encoding a protein implicated in retaining cytoplasmic proteins to the bacterial surface, was deleted, the yield of MVs production was 3-fold higher compared with WT. This suggests that absence of the yajC protein causes an of imbalance of the E. faecium cell wall, thereby impacting MV biogenesis in a positive way. However, in the mutant deprived of the major autolysin AtlA that hydrolyses the E. faecium cell wall, MV yield was similar to the WT strain. This suggests that autolysins may play a minor role in the MV production process in E faecium in contrast to the biogenesis of MVs in Staphylococcus aureus where autolysin can significantly promote the MV release by hydrolyzing the peptidoglycan layer.(Wang, Thompson et al. 2018) E. faecium has 8 different hydrolases that can catalyse the hydrolysis of peptidoglycan. (Paganelli, Willems et al. 2013) and the observation that muramidase-2 is highly abundant in the MVs might suggests a more important role of muramidase-2 in hydrolyzing peptidoglycan compared to the major autolysin used in this study.

Taken together, these data indicate that genetic engineering approach can be an approach to increase the production of MV by E. faecium.

Proteomics shows that MVs from each strain share a large set of proteins (120). In addition, each strain has their unique protein cargoes which differ from the others. In WT MVs, those proteins are related with translation, ribosomal structure and biogenesis. In yajC and AltA MVs, those proteins are related with amino acid/ carbohydrate transport and metabolism. Quantitative analysis results revealed that MVs from yajC contain more antigenic proteins (65.7\%) including murimidase-2, pbp5, LysM, AdcAfm 2410 and AdcAfm 2344 when compared with WT (58.5\%) and $\triangle$ AltA $(53.1 \%)$, which suggests that MV from WT and yajC may be MV-based vaccine candidate compared with AltA (Figure 4c). The vaccine candidates including PsaA, Pbp5, LysM, DdcP and PpiC are commonly present both in the MVs from E1162 strain in our study and also in the MVs from DO, E155, K60-39, K59-68 strains. (Wagner, Joshi et al. 2018) Drug resistance transporter EmrB and tellurite resistance protein TelA are also commonly present in MVs from E1162 and MVs from strain ATCC 700221. (Kim, Kim et al. 2019) 
The presence of glycopeptide resistance-related protein such as VanR-A, VanS-A was observed in previous study on the MVs from E155 strain (Wagner, Joshi et al. 2018), but these proteins are not present in the MVs from E1162 strain. This is logical as E1162 is a vancomycin-sensitive strain and four strains used in previous study are vancomycin-resistant. (McKenney, Yan et al. 2019)

To further investigate the potential of using the MVs from WT and its mutants as a vaccine platform. Healthy Balb/C mice were immunized by different administration route (sc and ip). No toxicity-associated symptoms were observed in these mice, and it was shown that both sc and ip immunization can trigger acute and longterm immune response. The cytokine study suggests that these MV product can increase both proinflammatory and anti-inflammatory cytokines. The observation of proinflammatory cytokine increase is also seen in a previous study by Kim et.al, in which it was reported that MVs from $E$. faecium can induce strong proinflammatory response in host cells. (Kim, Kim et al. 2019)

From a manufacture point of view, we provide culture and isolation methods which allows the harvest of clean, and quality-controllable MV products. As the gradientultracentrifugation isolation procedures may be difficult to be used for large volume of culture medium, further work towards rapid and time-effective, cost-effective methods should therefore be considered.

In summary, this study gives information how genetic engineering can influence the size, yield, protein compositions and immunogenicity of MVs from Gram-positive bacteria. The $\triangle y a j C$ and AltA genes play an important roles in the process of MV production. The high immunogenicity of MV from E1162 and its yajC mutant makes them promising and attractive vaccine candidates to fight infections caused by $E$. faecium.

\section{Materials \& methods}

\section{Bacterial strain and culture condition}

E. faecium strain E1162 (WT) and its isogenic mutants ( $\triangle$ yajC and $\Delta A(t A)$ were inhouse materials generated by Dr. Fernanda Paganelli.(Paganelli, Willems et al. 2013) To plot the growth curve, the WT strains were culture respectively in BHI, M1, TSB and LB medium, and the optical density $\left(\mathrm{OD}_{600}\right)$ were measured by Bioscreen $\mathrm{C}^{\mathrm{TM}}$ system every 15 min for 18 consecutive hours.

For the MVs used for characterization and the in vivo study, we harvested the MVs shed by bacteria in their exponential phase. 2-Step culturing procedures were applied. Firstly, a single colony was picked and inoculated overnight with aeration in $30 \mathrm{ml}$ 


\section{Chapter 5}

$\mathrm{BHI}$ medium at 37. Secondly, the overnight culture was spun down to remove the $\mathrm{BHI}$ medium and the bacteria pellet was resuspended in $30 \mathrm{~mL} \mathrm{M1}$ medium and scaled up in $3 \mathrm{~L} \mathrm{M1} \mathrm{medium} \mathrm{which} \mathrm{contained} \mathrm{30g} \mathrm{tryptone} \mathrm{(LP0042,} \mathrm{Oxoid} \mathrm{Ltd,} \mathrm{England),} \mathrm{1.5g}$ yeast extract (LP0021, Oxoid Ltd, England) and 1\% (m/V) glucose. OD 600 was closely monitored after 7-8 hours of culture at 37. To obtain samples with less batch-tobatch variation, MVs were harvested when bacteria were in their exponential phase of growth at an OD600 value of 0.55 .

\section{Isolation of the MVs}

To harvest the MVs, the culture suspensions were centrifuged twice at $4000 \times \mathrm{g}$ for 15 minutes at 4, and the supernatant was further filtered through an $0.45 \mu \mathrm{m}$ MCE membrane (Merck Milipore Ltd. Ireland). The obtained bacteria-free filtrate was concentrated with QuixStand ${ }^{\mathrm{TM}}$ Benchtop System which coupled with Xampler hollow fiber cartridges (GE Healthcare, Buckinghamshire, UK, HP7 9NA) to the final volume of approximately $200 \mathrm{~mL}$. This concentrate was further concentrated with 100kD Amicon ${ }^{\circledR}$ Ultra-15 Centrifugal Filters (Merck Millipore Ltd. Ireland) to final volume of $25 \mathrm{~mL}$.

The crude MVs in the concentrate were further pelleted by ultracentrifuge at 40.000 rpm for $2 \mathrm{~h}$ at $4{ }^{\circ} \mathrm{C}$ (Beckman Coulter Optima ${ }^{\mathrm{TM}}$ L-90K with fix-angle rotor $50.2 \mathrm{Ti}$ ).

In order to purify the crude MV pellet, density gradient ultracentrifugation (DGC) was applied. In brief, the MV crude pellet was resuspended in pre-chilled $60 \%$ OptiPrep solution (Sigma-Aldrich. Germany) and loaded on the bottom. Different concentrations of OptiPrep solutions were prepared (55\%, 50\%, 45\%, 40\%, 35\%, $30 \%, 25 \%, 20 \%, 15 \%, 10 \%$ and $0 \%$ in PBS) and overlaid on top of the $60 \%$ fraction. Ultracentrifugation was carried out at $39.000 \times \mathrm{rpm}$ for 14 hours at $4^{\circ} \mathrm{C}$ with Optima XE-90 Ultracentrifuge (Beckman Coulter. USA) together with swing-out rotor SW $40 \mathrm{Ti}$. After ultracentrifugation, the different fractions were collected into sterile Eppendorf tubes.

Fractions containing MVs (15\%-30\%) were pooled and concentrated with $100 \mathrm{kD}$ Amicon centrifugal filter (Merck Millipore, Cork Ireland) at $4000 \times \mathrm{g}$ for $5 \mathrm{~min}$ at $4{ }^{\circ} \mathrm{C}$. The MV concentrate was washed with $7.5 \mathrm{ml}$ of ice-chilled PBS for 3 times in Amicon centrifugal filter to remove the iodixanol.

\section{SDS- PAGE and silver staining}

Proteins present in the different MV samples were separated by SDS-PAGE. In brief, $20 \mu \mathrm{L}$ of each MV sample Optiprep fraction (MVs were isolated from $3 \mathrm{~L}$ culture of bacteria at the OD 0.55) was mixed with $3 \times$ sample buffer with $25 \mathrm{mM}$ dithiothreitol (DTT), and the mixtures were boiled for 10 minutes at $90^{\circ} \mathrm{C} . \mathrm{MVs}$ from WT, yajC and 
AltA were loaded onto a 4-12\% Bis-Tris gel (Invitrogen, Thermo Fisher Scientific, Life Technology, USA). Subsequently, the proteins were separated by electrophoresis at the voltage of $100 \mathrm{~V}$ (Biorad).

To visualize the protein compositions. a sensitive silver-staining method was used. The gel was firstly fixed in fixative solution (45\% methanol-20\% acetic acid in milliQ water) for $30 \mathrm{~min}$ at room temperature with gentle shaking. Afterwards. the gel was incubated for $30 \mathrm{~min}$ with incubation solution (ethanol/sodium-thiosulphate/sodiumacetate/glutaradehyde/milliQ water). Next, the gel was washed 3 times with superQ water and further incubated with staining solution (silver nitrate/formaldehyde/miliQ water) for $40 \mathrm{~min}$ at room temperature while keep the gel in dark. Subsequently, the gel was rinsed with milliQ water to remove excess staining solution. To visualize the protein patterns, the gel was then in incubated in developing solution (sodium carbonate/formaldehyde/miliQ water) until clear visible bands appeared. In the end, the reaction was terminated by bringing the gel in stop solution (EDTA/miliQ water) and finally stored in milliQ water. The silver-stained gel was imaged by HP Scanjet 4850 scanner.

\section{Concentration and size distribution of the isolated MVs}

MVs were isolated from 3L M1 culture of each bacteria (OD 0.55). And the purified MVs were normalized to equal final volume for size and concentration measurement.

To determine the yield and size of the isolated MVs, nanoparticle tracking analysis (NTA) was performed with a NanoSight NS500 system equipped with an LM14 405 nm violet laser unit (Malvern Instruments. UK). The three MV samples were diluted in cold, sterile PBS to appropriate concentrations before analysis. Three different movies of $30 \mathrm{~s}$ were recorded, and the camera level was set at 13 which included acquisition of 3 movies of $1 \mathrm{~min}$ at a fixed temperature of $22^{\circ} \mathrm{C}$. The concentration and size were analyzed using the software NTA version 3.3. The detection threshold was set at 6 and analysis was performed with NTA 3.1 software.

\section{Negative staining transmission electron microscopy}

To image MVs, $20 \mu \mathrm{L}$ of each MV fractions was first incubated with copper grids which were freshly coated with carbon for 5 minutes. Afterwards. MV-absorbed sample was fixed with $1 \%$ glutaraldehyde in PBS for 10 min. Next the grids were intensively washed for 8 times with distilled water to remove excess fixative and salt. Finally. the grids were stained with Uranyl cellulose $\mathrm{pH} 4.0$ for $5 \mathrm{~min}$ on ice, the excess staining solution was removed using a filter paper and the grids were dry in air. The grids were then visualized with a transmission electron microscope (FEI. Technai 12). 


\section{Chapter 5}

\section{Cryo- electron microscopy}

To image the intact morphology of MV samples, $20 \mu \mathrm{L}$ of each MV fractions was first incubated with copper grids which were freshly coated with carbon. The MV samples deposited on the grids were fixed and then plunged into liquid ethane. Next, the grids were transferred into a Gatan cryo-sample holder and store in liquid nitrogen before imaging. All images were acquired by TEM (FEI, Technai 12).

\section{TCA protein precipitation}

Density gradient fractions $15-30 \%$ were pooled and trichloroacetic acid (TCA) protein precipitation procedures were applied. In brief, pure TCA and 2\% deoxycholate were added to MV suspension to a final concentration of $10 \%$ and $0.02 \%$, respectively, to precipitate the proteins. Afterwards, the mixture were centrifuged at $16000 \mathrm{~g}$ for 15 $\min$ at $4^{\circ} \mathrm{C}$ and the supernatants were removed. The pellets were then washed with $500 \mathrm{l}$ ice-cold acetone and again centrifuged at $16000 \mathrm{~g}$ for $5 \mathrm{~min}$, the supernatants were discarded and the samples were dried at room temperature for $5 \mathrm{~min}$.

\section{Proteomics}

Proteins were reconstituted in lysis buffer ( $100 \mu \mathrm{L} 1 \mathrm{M}$ TEAB buffer $\mathrm{pH} 8.5$ was added to $480 \mathrm{mg}$ urea and water was added to a final volume of $1 \mathrm{ml}$ ). Next $50 \mathrm{mg}$ sodium deoxycholate was added and the detergent was dissolved by vortex. Afterwards, 20 $\mu \mathrm{L}$ lysis buffer was added to the TCA-precipitated protein samples for downstream proteomics.

Digestion of proteins was done according to a suggested powerful. reproducible. sample-saving, and cost-effective protocol. (Schniers, Pasing et al. 2019) OMIX C18 tips (Varian. Inc. USA) was used for sample cleanup and concentration. Peptide mixtures $(2 \mu \mathrm{g})$ containing $0.1 \%$ formic acid were loaded onto a Thermo Fisher

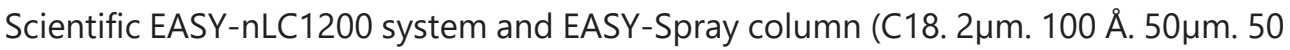
$\mathrm{cm})$. Peptides were fractionated using a $4-40 \%$ gradient of increasing amounts of $80 \%$ acetonitrile in water over $120 \mathrm{~min}$ at a flow rate of $300 \mathrm{nl} / \mathrm{min}$. The mobile phases contained $0.1 \%$ formic acid. Separated peptides were analyzed using an Orbitrap Fusion Lumos (Thermo) mass spectrometer. The mass spectrometer was operated in a data-dependent mode with the precursor scan in the orbitrap over the range $\mathrm{m} / \mathrm{z}$ 375-1500. The most intense ions were selected for CID fragmentation using $3 \mathrm{sec}$ between each master scan. Dynamic exclusion was set to 60s. The Orbitrap AGC target was set to $4 \mathrm{E} 5$ and the MS2 scans in the lon Trap was set to $3 \mathrm{E} 3$ with maximum injection times 50 and $300 \mathrm{~ms}$. respectively. Raw data were processed using MaxQuant ( $v$ 1.6.0.16) with the integrated Andromeda search engine. MS/ MS data were searched against the E1162 protein database which was generated based on the E1162 whole-genome sequencing data. A FDR ratio of 0.01 was needed 
to give a protein identification. Protein quantitation by LFQ and iBAQ was done in Perseus (v 1.6.0.7). For LFQ quantitation. LFQ intensities were Log10 transformed. Rows were filtered based on min 3 valid values. Missing values were replaced with a value from the normal distribution with a down shift of 1.8. Hierarchical clustering and volcano plots were produced in Perseus. For iBAQ quantitation, a relative iBAQ value (riBAQ) was calculated as riBAQ $=\mathrm{iBAQ} / \Sigma i B A Q \times 100$.

Analyses to generate the Venn diagram were performed in $\mathrm{R}(\mathrm{v} 3.5 .1)$ with the package VennDiagram.

\section{In vivo experiment to evaluate the released cytokine level and IgG level}

Mouse experiments were carried out within the guidelines of IvD Utrecht. Animal Welfare Body Utrecht. Boost immunization was given to the subject mice every 2 weeks within the total experimental course of 6 weeks. In each immunization, $5 \mathrm{~g}$ of each MV samples (quantify by MVs total proteins) in 100L PBS (mock isolation MV. yajC and AltA) were injected into BALB/c mice ( $n=5$. Charles River Laboratories) either subcutaneously or intraperitoneal. Plasma samples were collected before each injection by cheek puncture. For short-term immune response experiment, extra plasma was collected after $18 \mathrm{~h}$ at week 4 . At the endpoint of the experiment (after 6 weeks), the mice were sacrificed and blood was withdrawn by heart puncture. AltA strain was not included in the study because these vesicles were obtained and in too low yield.

\section{Olink and data analysis}

The collected mice plasma samples were subjected to Olink mouse exploratory screening. Olink mouse exploratory offers 92 high-quality assays for murine proteins. The proteins assayed in the panel are all known to be detectable in human serum and plasma, increasing the human relevance of the findings with the mice. Samples of $2 \mathrm{~L}$ plasma were analyzed with proximity extension assay (PEA), which utilizes pairs of protein specific oligonucleotide-linked antibodies and involves qPCR to detect and quantify target proteins. The proteins' levels were expressed with linear NPX (Normalised Protein eXpression), which is a relative quantification scale in arbitrary units. (https://www.olink.com/)

For calculation of the cytokine expression level in plasma, the cytokine level was converted into linear scale, and the average values were calculated for the different groups (mock, WT and yajC) at time point 1) 4 week and 2) 4 week $+18 \mathrm{~h}$. Data at time point 4 week served as the background, and the protein level was substrate by this background value. If the protein level in WT and yajC group was 2 fold higher than the mock group, this proteins were send to STRING database (https://string-db.org/) which is used for identification of protein-protein interactions. 


\section{Chapter 5}

\section{Enzyme-linked immunosorbent assay (ELISA)}

WT single colony in $\mathrm{BHI}$ medium were inoculated for $8 \mathrm{~h}$, and bacteria were pelleted at $2000 \mathrm{~g}$ for $5 \mathrm{~min}$ and washed twice with PBS solutions. Next, $5 \times 10^{6}$ bacteria suspendend in $50 \mu \mathrm{L}$ were added to each well on a high-binding ELISA plate (like Maxisorp plate), and incubated overnight at $4^{\circ} \mathrm{C}$. The coating bacteria suspension was washed 3 times with $100 \mu \mathrm{L}$ washing buffer ( $0.05 \%$ Tween $20 / \mathrm{PBS})$, and then blocked with $300 \mu \mathrm{L}$ Block Solution (0.05\% Tween20/ PBS/ 4\%BSA) for $1 \mathrm{~h}$ at $37^{\circ} \mathrm{C}$. Then, the blocking buffer was washed 3 times with $100 \mu \mathrm{L}$ washing Buffer and incubated with $50 \mu \mathrm{L}$ serum in dilutions of $1: 10,1: 100$ and $1: 1000$ at $37^{\circ} \mathrm{C}$ for $1 \mathrm{~h}$. The plasma samples were washed 3 times with washing buffer and incubated with secondary antibody peroxidase labeled Goat-anti-Species-lgG (1:6000, southern Biotech) at $37^{\circ} \mathrm{C}$ for $1 \mathrm{~h}$. The secondary antibody were washed with $100 \mu \mathrm{L}$ washing buffer for 3 times.

For detection, $50 \mu \mathrm{L}$ TMB substrate solution was added to each well and incubated at $\mathrm{RT}$ the solution became blue $(15 \mathrm{~min})$, and the reaction was terminated with $50 \mu \mathrm{L}$ $\mathrm{H}_{2} \mathrm{SO}_{4}(1 \mathrm{M})$. Finally, the ODs of the samples at $450 \mathrm{~nm}$ were measured and plotted with GraphPad Prism 8.3.

\section{Statistics}

One-way ANOVA with Tukey's multiple comparison test was applied for the analysis of MV size, particle concentrations and IgG levels in mice plasma. 


\section{Reference}

Brown, L., et al. (2015). "Through the wall: extracellular vesicles in Gram-positive bacteria, mycobacteria and fungi." Nature Reviews Microbiology 13(10): 620-630.

Collins, B. S. (2011). "Gram-negative outer membrane vesicles in vaccine development." Discovery medicine 12(62): 7-15.

Haurat, M. F., et al. (2015). "Prokaryotic membrane vesicles: new insights on biogenesis and biological roles." Biological chemistry 396(2): 95-109.

Heikens, E., et al. (2007). "Enterococcal surface protein Esp is important for biofilm formation of Enterococcus faecium E1162." Journal of bacteriology 189(22): 8233-8240.

https://www.olink.com/.

https://www.uniprot.org/proteomes/UP000004426.

Kim, M. H., et al. (2019). "Production of membrane vesicles by Enterococcus faecium cultured with or without subinhibitory concentrations of antibiotics and their pathological effects on epithelial cells." Frontiers in cellular and infection microbiology 9: 295.

Kropec, A., et al. (2011). "Identification of SagA as a novel vaccine target for the prevention of Enterococcus faecium infections." Microbiology 157(12): 3429-3434.

Kulp, A. and M. J. Kuehn (2010). "Biological functions and biogenesis of secreted bacterial outer membrane vesicles." Annual review of microbiology 64: 163-184.

Lässer, C., et al. (2018). "Subpopulations of extracellular vesicles and their therapeutic potential." Molecular aspects of medicine 60: 1-14.

McKenney, P. T., et al. (2019). "Intestinal bile acids induce a morphotype switch in vancomycin-resistant Enterococcus that facilitates intestinal colonization." Cell host \& microbe 25(5): 695-705. e695.

Paganelli (unpublished data). "unpublished data."

Paganelli, F. L., et al. (2013). "Enterococcus faecium biofilm formation: identification of major autolysin AtIAEfm, associated Acm surface localization, and AtIAEfm-independent extracellular DNA Release." MBio 4(2): e00154-00113.

Roier, S., et al. (2016). "A novel mechanism for the biogenesis of outer membrane vesicles in Gramnegative bacteria." Nature communications 7: 10515.

Romero-Saavedra, F., et al. (2015). "Characterization of two metal binding lipoproteins as vaccine candidates for enterococcal infections." PLoS one 10(8).

Romero-Saavedra, F., et al. (2014). "Identification of peptidoglycan-associated proteins as vaccine candidates for enterococcal infections." PLoS one $\mathbf{9}(11)$.

Schniers, A., et al. (2019). Lys-C/Trypsin Tandem-Digestion Protocol for Gel-Free Proteomic Analysis of Colon Biopsies. Proteomics for Biomarker Discovery, Springer: 113-122.

Sinnige, J. C., et al. (2015). "Growth condition-dependent cell surface proteome analysis of Enterococcus faecium." Proteomics 15(22): 3806-3814.

Toyofuku, M., et al. (2019). "Types and origins of bacterial membrane vesicles." Nature Reviews Microbiology 17(1): 13-24.

Wagner, T., et al. (2018). "Enterococcus faecium produces membrane vesicles containing virulence factors and antimicrobial resistance related proteins." Journal of proteomics 187: 28-38.

Wang, X., et al. (2018). "Release of Staphylococcus aureus extracellular vesicles and their application as a vaccine platform." 9(1): 1379.

Yáñez-Mó, M., et al. (2015). "Biological properties of extracellular vesicles and their physiological functions." Journal of extracellular vesicles 4(1): 27066.

Zhang, X., et al. (2012). "Genome-wide identification of ampicillin resistance determinants in Enterococcus faecium." PLoS genetics 8(6). 


\section{Chapter 5}

\section{Acknowledgement}

Dr. Fatemeh Askarian (Faculty of Chemistry, Biotechnology and Food Science, Norwegian University of Life Sciences) was acknowledged for setting up the proteomics collaboration; Mr. Cor Seinen (CDL Research, Division LAB, Faculty of Medicine, University Medical Center Utrecht, the Netherlands) is acknowledged for kindly assistance with the negative EM staining and cryo-EM experiment; Mr. JackAnsgar Bruun (UiT Norges arktiske universitet) and Toril Anne Grønset (UiT Norges arktiske universitet) are acknowledged for performing quantitative and qualitative proteomics study and data analysis; Mr. Arjan Schoneveld (CDL Research, Division LAB, Faculty of Medicine, University Medical Center Utrecht, the Netherlands) is acknowledged for Olink study. 


\section{Supplementary Information}

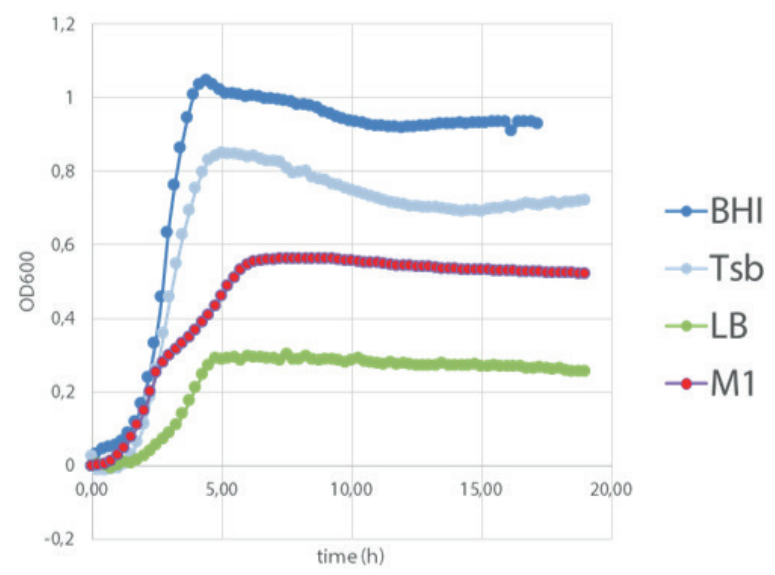

FIGURE S1. Growth rate of WT in different media.

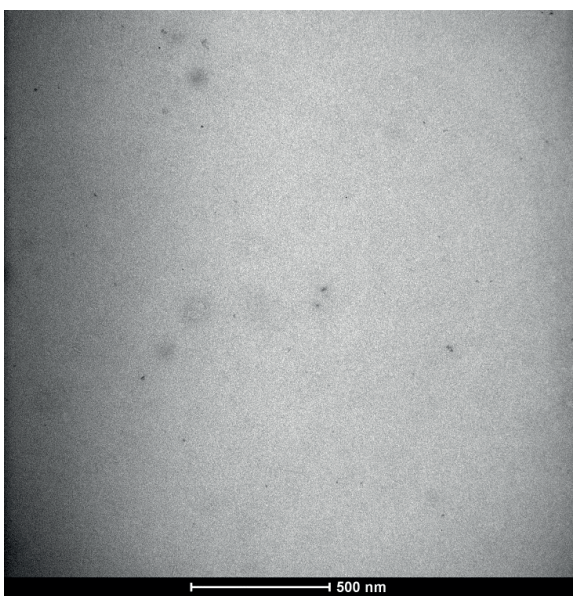

FIGURE S2. TEM photograph of purified MVs from E1162 cultured in TSB medium. 
Chapter 5

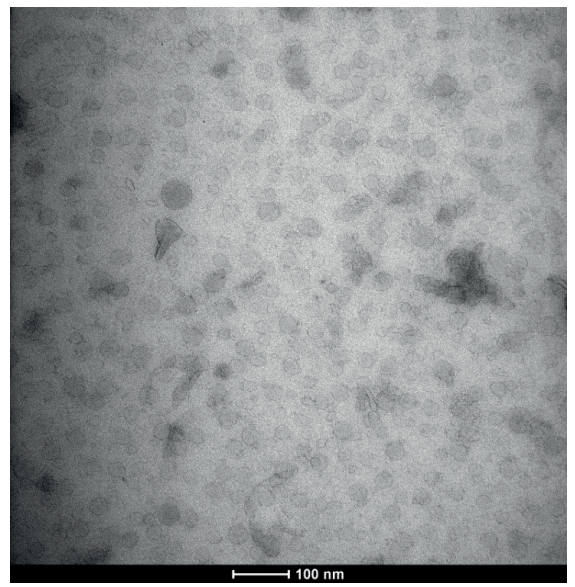

FIGURE S3. TEM photograph of MVs from EV-depleted BHI medium.

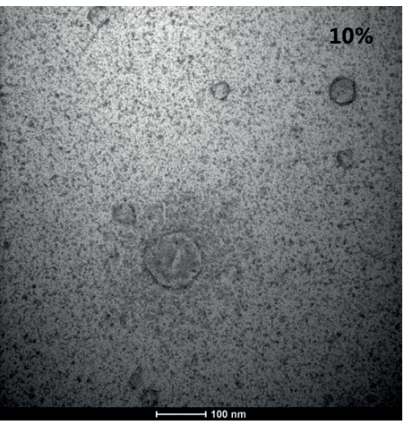

(a)

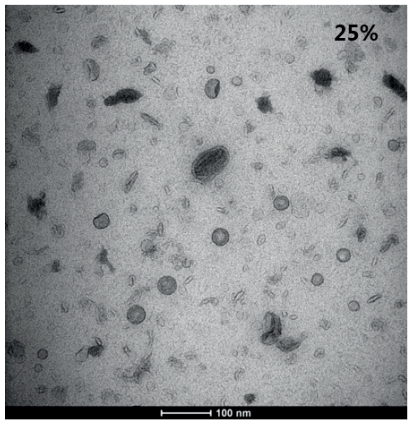

FIGURE S4. TEM pictures of MVs from E1162 WT strain were purified by density gradient ultracentrifugation (Optiprep), and MV's from different fractions (10\%-35\%) were visualized by TEM (a-g). 


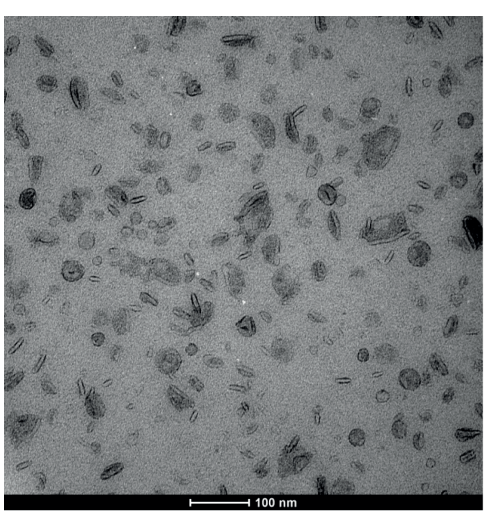

(a)

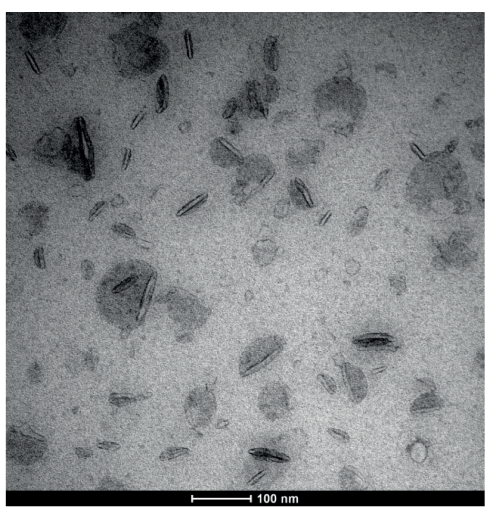

(b)

FIGURE S5. TEM photographs of the MVs from yajC (a) and AltA (b).

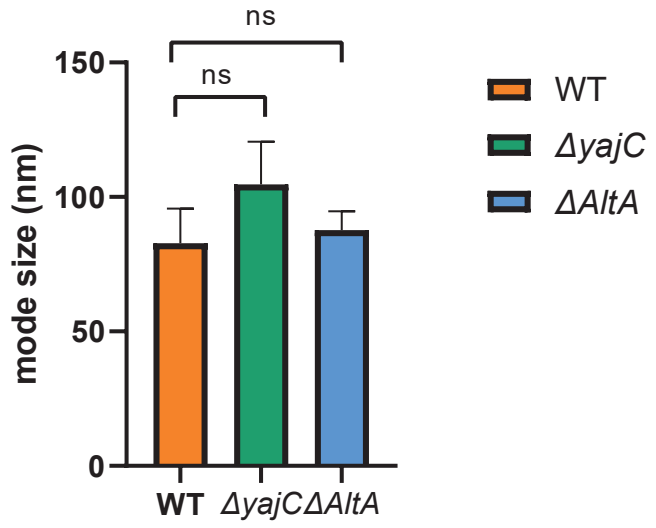

FIGURE S6. Mode size of MVs from WT and its mutants measured by NTA (standard error $= \pm 25 \mathrm{~nm}$, $n=3)$. 
Chapter 5

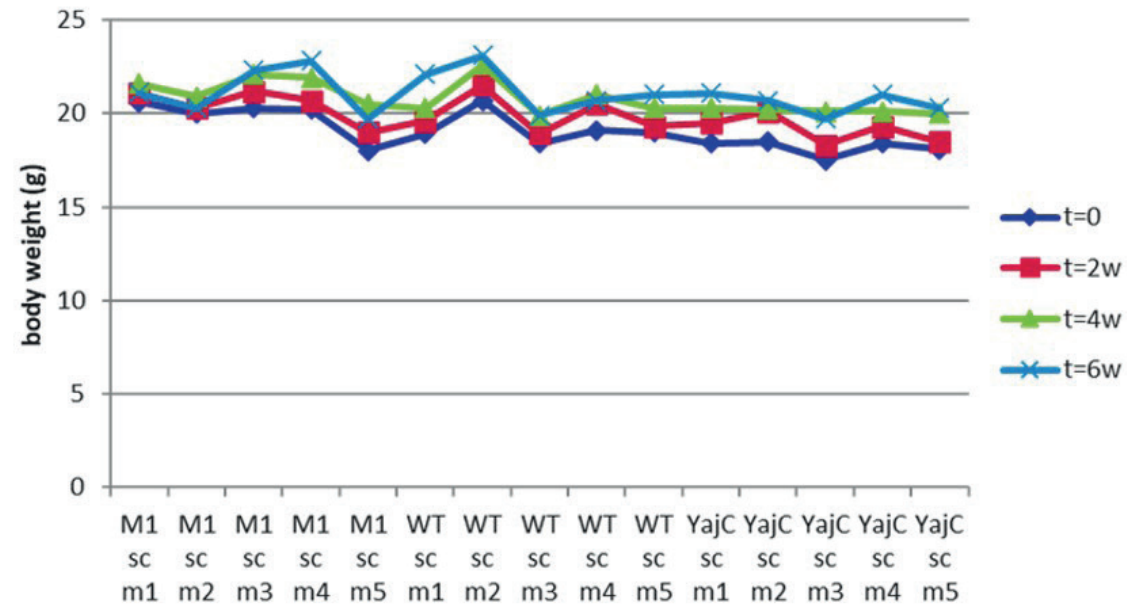

(a)

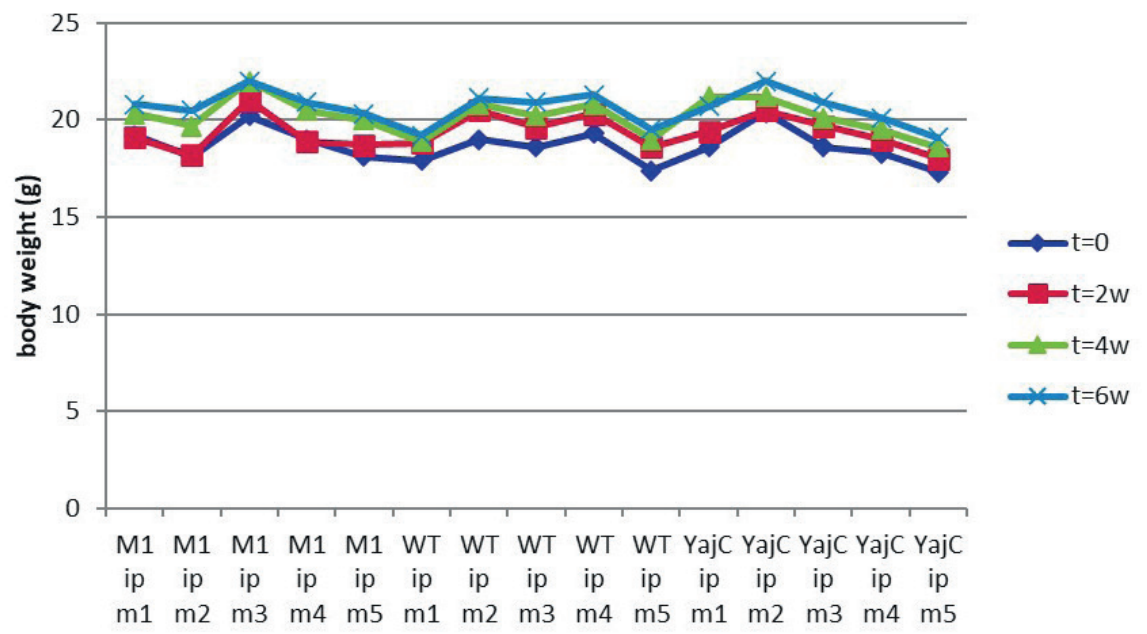

(b)

FIGURE S7. Bodyweight of mice within the experiment course. 


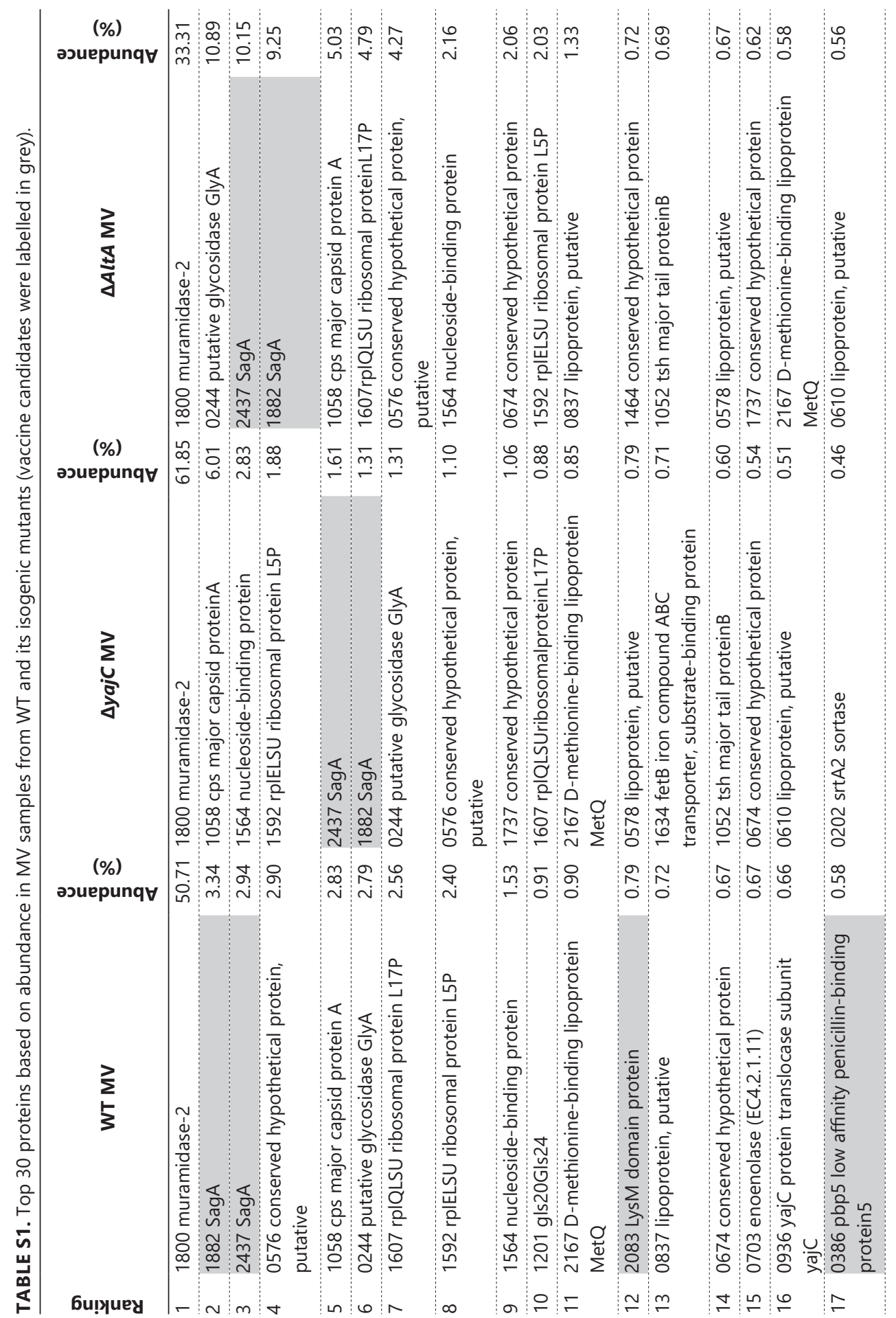




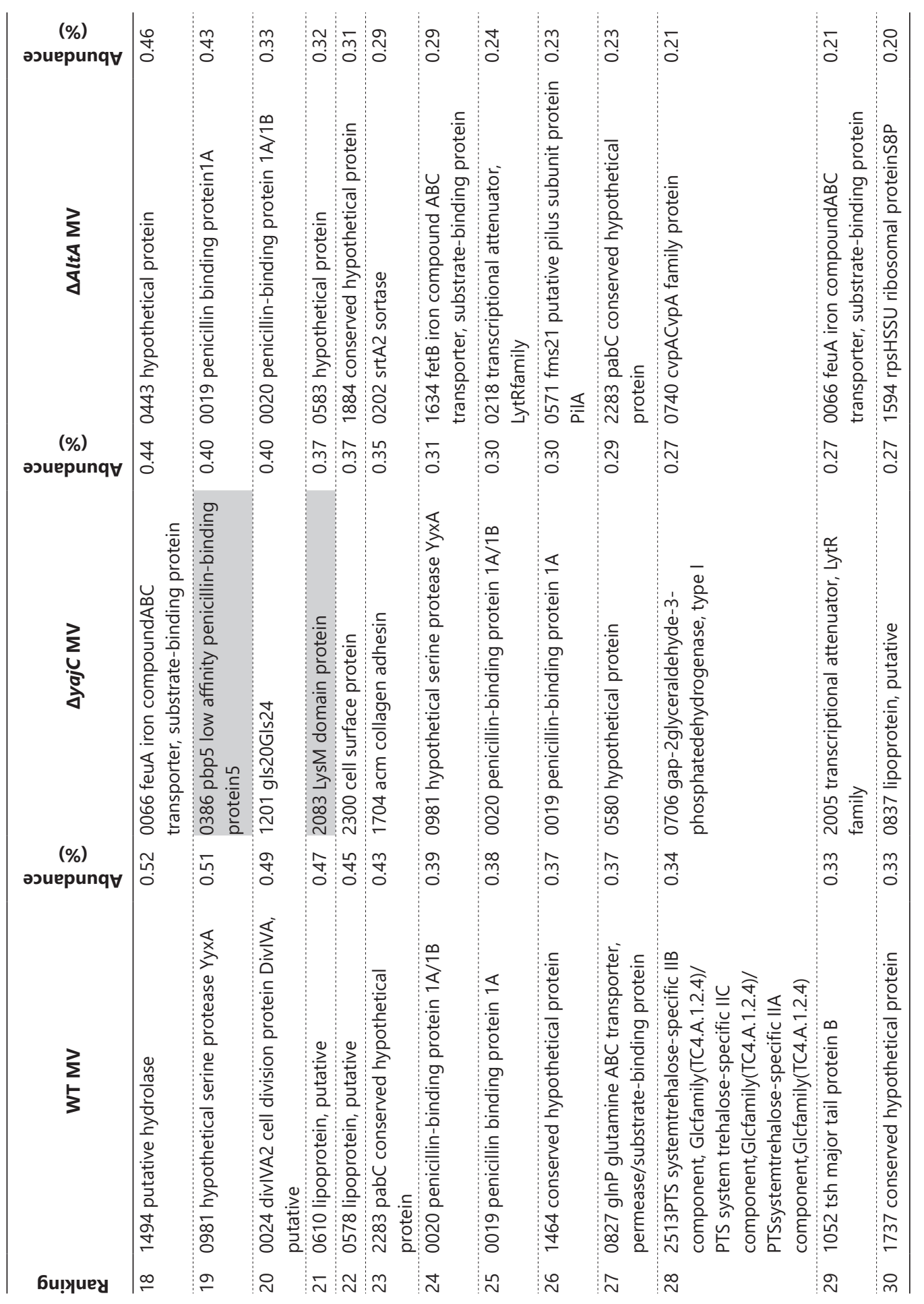


Membrane Vesicles for combating E. faecium infection

TABLE S2. Abundance of proteins (\%) in MVs based vaccine candidates.

\begin{tabular}{|c|c|c|c|}
\hline & WT & $\Delta$ yajC & $\Delta$ AltA \\
\hline muramidase -2 & 50.70 & 61.80 & 33.30 \\
\hline SagA 1882 & 3.30 & 1.30 & 9.20 \\
\hline SagA 2437 & 2.90 & 1.60 & 10.20 \\
\hline pbp5 & 0.60 & 0.40 & 0.10 \\
\hline LysM & 0.78 & 0.37 & 0.16 \\
\hline DdcP & 0.06 & 0.00 & 0.01 \\
\hline PpiC & 0.10 & 0.14 & 0.12 \\
\hline PsaAfm & 0.03 & 0.02 & 0.00 \\
\hline AdcAfm 2410 & 0.02 & 0.02 & 0.01 \\
\hline AdcAfm 2344 & 0.03 & 0.02 & 0.01 \\
\hline Sum of the vaccine candidate proteins & 58.51 & 65.67 & 53.11 \\
\hline Other proteins & 41.49 & 34.33 & 46.89 \\
\hline
\end{tabular}




\section{Chapter 6}

\section{A post-insertion strategy for surface functionalization of bacterial and mammalian cell-derived extracellular vesicles}

Linglei Jiang ${ }^{1}$, Joen Luirink ${ }^{2,3}$, Kok P. M. van Kessel ${ }^{4}$, Wouter Jong ${ }^{3}$, Max van Essen', Cor Seinen', Olivier de Jong', Jerney Francois-Gitz' ${ }^{1}$ Pieter Vader ${ }^{1,5}$ and Raymond M. Schiffelers ${ }^{1 *}$

1. Laboratory of Clinical Chemistry and Hematology, UMC Utrecht, Utrecht, the Netherlands

2. Department of Molecular Microbiology, Amsterdam Institute for Molecules Medicines and Systems (AIMMS), Vrije Universiteit, Amsterdam, Netherlands

3. Abera Bioscience AB, Solna, Sweden

4. Medical Microbiology, UMC Utrecht, Utrecht, the Netherlands

5. Laboratory of Experimental Cardiology, UMC Utrecht, Utrecht, the Netherlands

* Corresponding author

E-mail address: r.schiffelers@umcutrecht.nl (R.M. Schiffelers) 


\section{Chapter 6}

\section{Abstract}

Extracellular vesicles (EVs) are nanoparticles which can be released by organisms from different domains such as mammalian cells, bacteria and fungi etc. They mediate cell-cell communication by transferring the information cargoes such as proteins and nucleic acids. EVs receive great interest in both academia and industry as they have the potential to be natural drug carriers or vaccine candidates. However, the limitations of their clinical translation do exist as lacking of efficient isolation and labelling methods. And some EV types exhibit short circulation time in vivo, or has difficulty to reach the site of disease or has poor immunogenicity. In this article, we investigate a 'post-insertion' approach which is commonly used in the functionalization of liposome in pharmaceutical field on two different EV types mammalian cell-derived $\mathrm{EVs}$ and bacteria-derived $\mathrm{EVs}$, aiming to find an easy and flexible approach to functionalize them, and thus improving the efficiency regarding their labelling and isolation. In addition, to increasing their targeting and immunogenicity capabilities. 


\section{Introduction}

Extracellular vesicles (EVs) are nanosized phospholipid bi-layered membrane-bound structure which can be released by organisms from different domains including eukaryote, prokaryote, archaea and fungi. The main compositions of EVs are proteins, lipids and nucleic acids. They are important signaling entities that mediate intercellular communication. And they also represent a promising drug carrier platform due to the fact that they can protect encapsulated drugs (such as protein and RNA) from degradation. In addition, they have the potential to target specific tissues such as solid tumors or myocardial infarction sites via enhanced permeability effect (EPR). (Torchilin 2011, Nguyen, Carlini et al. 2015) In addition, EVs hold therapeutic potential as vaccine candidates. For example,immune-stimulatory molecules such as MHC class I and II complexes are present on the surface of dendritic cells (DCs), and MHC I and II complexes are also present on the surface of their derived EVs. From a technical point of view, DCs can be expanded in vitro condition, so it can be manufactured at large-scale level.(Zitvogel, Regnault et al. 1998) Pathogen-associated molecular pattern molecules (PAMPs) are present on the surface of bacteria, and the PAMPS therefore also present on the surface of EVs shed from these bacteria.(Fais, O'Driscoll et al. 2016, Jiang, Schinkel et al. 2019) As EV vaccine is non-replicate, it is safer for clinical use when compared with inactivated bacteria vaccine. Due to these merits EVs have for cell-cell communication, for drug carrier and vaccination purpose, EVs have triggered interest in both academia and industry.

However, challenges do exist to advance EV research. For example, efficient labelling approach is lacking when studying the biological roles of EVs in physiology and pathology. Traditional labelling methods using lipidic dyes (e.g. PKH, DiO, DiR) might result in undesirable dye aggregates or micelles and hence may introduce 'labelling artifacts'. On the other hand, genetic modification of donor cells for labelling purposes (e.g. fuse palmtdTomato fluorescent marker to EV surface protein CD63) may limit the tracking only to certain subtypes of EVs.(Chuo, Chien et al. 2018) In addition, the circulation time for some specific EV types may not be long enough for therapeutic purpose. And 'off-target effect' to the site of disease may exist in clinical application. At last, current isolation approaches are usually time-consuming and laborious which hinder the further scale-up in manufacture. Together, these obstacles necessitate the development of a platform that would allow efficient EV labelling, rapid isolation and addition of targeting ligands.

A membrane post-insertion approach could be considered as an attractive strategy for EV modification. 'Post-insertion' is a simple and flexible method commonly used for producing ligand-coupled liposomes. In this method, ligands of interest are firstly covalently linked to polyethylene glycol (mPEG)-lipid micelles, and afterwards 


\section{Chapter 6}

incubate the ligand-PEG conjugate with liposome of choice, the ligand then can be transferred to liposome under desirable physiochemical condition. (Allen, Sapra et al. 2002)

Previous reports have shown that by incubating mammalian cells in medium containing lipid conjugates distearoyl phophatidylethanolamine-poly(ethylene glycol)-biotin (DSPE-PEG-biotin) or DSPE-PEG-folate (DSPE-PEG-FA) micelles, leads to spontaneous incorporation into the plasma membrane. As a result, EVs shed from these donor cells are also modified. (Zhang, Yu et al. 2017, Zhu, Dong et al. 2017) Using this 'post-insertion' approach, decoration of the EV membrane with biotin as well as FA is enabled. Several applications and advantages of this approach can be envisioned: 1) as DSPE-PEG lipids form micelles in aqueous solution, it is unlikely to aggregate when compared with traditional lipidic dyes; 2)EVs modified with PEG anchors have been shown to display prolonged circulation times in vivo and thus have increased opportunity to accumulate in target tissue.(Kooijmans, Fliervoet et al. 2016) 3) Successfully biotinylated EVs can bind to a number of streptavidin-conjugated entities such as streptavidin-dye conjugates, streptavidincoated magnetic beads, and streptavidin-coupled antigens/antibodies, which offers opportunities for lipid dye-free, specific labelling, rapid isolation (compare with traditional ultracentrifugation methods) and flexible modification of the immunogenicity of EVs. In addition, successful incorporation of FA moieties onto the surface of EVs can enhance their targeting to the FA receptor highly expressed cells, for example, tumor cells. Previous reports show an efficient post-insertion labelling of two cell types under single culture conditions.(Zhang, Yu et al. 2017) Here we investigated the robustness of the labelling in additional mammalian cell lines grown under different conditions. In addition, as bacteria-derived vesicles have emerged as a promising natural nanomedicine platform for vaccination strategies, we also tested the same modification approach here. This is the first time that a 'post-insertion' strategy is being used for bacterial outer membrane vesicles (OMVs) to our knowledge.

\section{Results}

Post-insertion labelling efficiency of MDA-MB-231 and HEK 293T cells can be improved when cells are cultured in suspension mode and longer period of time

In order to improve the strategy for mammalian cells, two different cell types, i.e. a breast cancer cell line MDA-MB-231 and a human embryonic kidney cell line HEK 293T, were cultured with/without $5 \mu \mathrm{g} / \mathrm{mL}$ DSPE-PEG-biotin lipid micelles in either adherent mode or suspension mode for 2 and 5 days, respectively. After 2-day culture, cells were collected after removing the free lipid micelles by centrifugation 


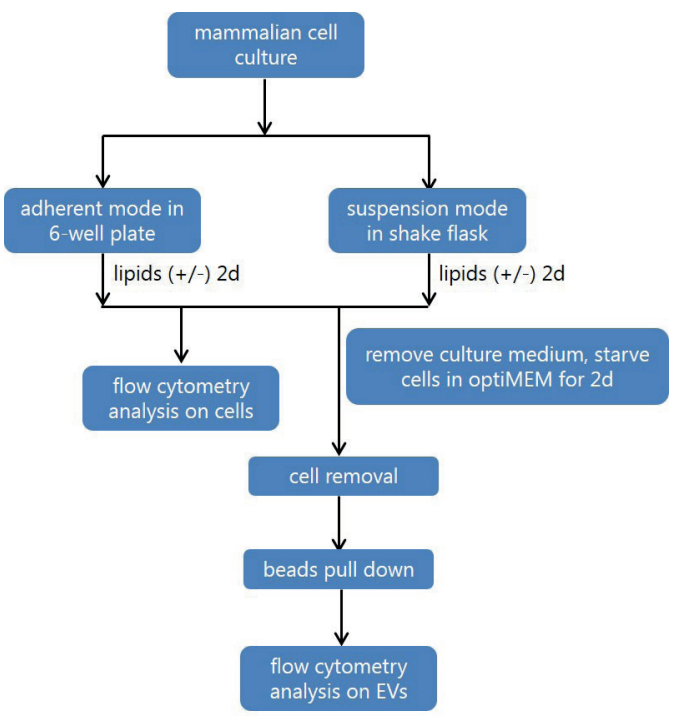

(a)

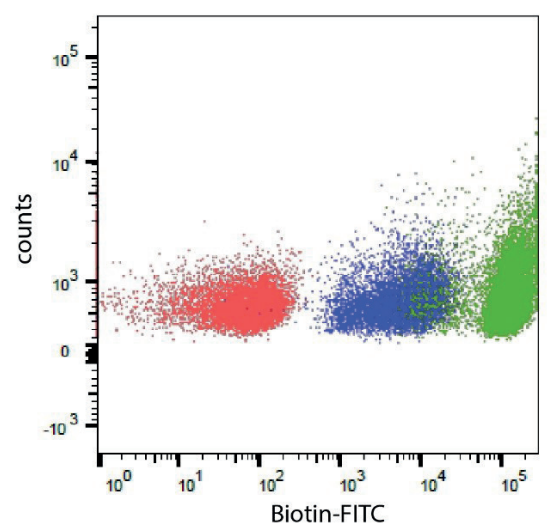

MDA-MB-231 cell DSPE-PEG-biotin (-)

MDA-MB-231 cell DSPE-PEG-biotin (+)/ adherent

MDA-MB-231 cell DSPE-PEG-biotin (+)/ suspended

(b)

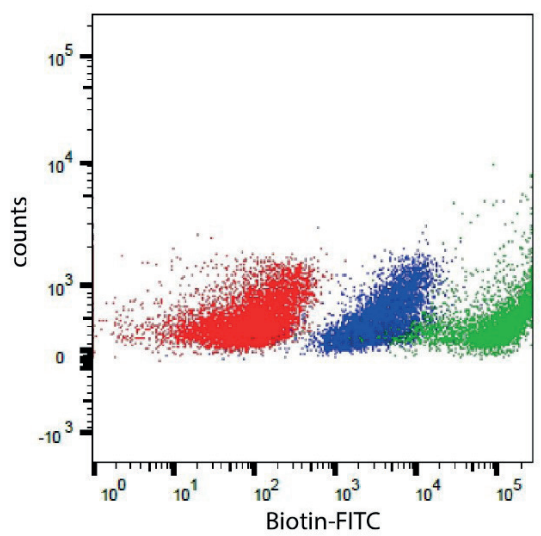

HEK 293T cell DSPE-PEG-biotin (-)

HEK 293 T cell DSPE-PEG-biotin (+) / adherent

HEK 293 T cell DSPE-PEG-biotin (+) / suspended

(c)

FIGURE 1. Schematic diagram of cell cultured in adherent mode and suspended mode (a). Flow cytometic quantification of biotin on the surface of MDA-MB-231 cells (b) and HEK 293T cells (c) cultured in adherent and suspended mode for 2 days.

and filtration. The collected cells were then further divided to two groups. One group of cells were subjected to fluorescent-labelling with streptavidin-FITC and then 


\section{Chapter 6}

analyzed by flow cytometry, the other group of cells were further starved in EV-free OptiMEM medium for additional 2 days to produce EVs for further analysis. (Figure 1a) Both MDA-MB-231 and HEK 293T cells exhibited a more pronounced fluorescent shift when they were cultured in suspension mode compared to adherent mode (Figure $1 \mathrm{~b}$ and $1 \mathrm{c}$ ), which suggest higher labelling efficiency can be achieved when the cells are cultured in suspension. After culturing for 5 days in adherent mode, both MDA-MB-231 and HEK 293T cells showed more fluorescent labelling than the cells incubated for 2 days (Figure S1a and S1c), which suggests the lipids assemble into the membrane of cells in a time-dependent manner. This time-dependent increase was also seen in MDA-MB-231 cells when cultured in suspension mode (Figure S1b).

Post-insertion labelling efficiency of MDA-MB-231 cells-derived EV can be improved when cells are cultured in suspension mode but not HEK $293 T$ cells-derived EV

Next, to investigate the labelling efficiency of EVs derived from these cells, both cell types were first cultured in adherent and suspended mode in the presence of DSPEPEG-biotin after which they were starved in serum-free OptiMEM medium for 2 days. As CD63 is a general protein marker expressed on the mammalian EVs, a CD63magnetic bead pull-down assay was performed. In this assay, the CD63-expressing EVs are bound to the magnetic CD63-antibody-carrying beads. CD63-positive EVs that are bound to the beads can be enriched on a magnetic plate within 5 mins. The captured EVs were then fluorescently labeled with streptavidin-FITC conjugate. (Figure 4a)

For MDA-MB-231 cells, when cultured in adherent mode, their EVs showed no labelling based on FACS analysis (Figure 2a). In contrast, for cells cultured in suspended mode, EVs display clear fluorescent labelling (Figure 2b). This suggests that the suspension culture can contribute to a higher EV labelling efficiency by this post-insertion strategy. In contrast, EVs from HEK $293 \mathrm{~T}$ cells show a more pronounced fluorescent label in adherent mode then suspended mode (Figure $2 c$ and $2 \mathrm{~d}$ ). Combined, cell culturing conditions strongly influence the post-insertion efficiency in both mammalian cells and their EVs. For cells, higher labelling efficiency is achieved for the HEK 293 and MDA-MB-231 cells when cultured in suspension mode compared with the adherent mode reported previously. The labelling efficiency for EVs does not correlate with the labeling efficiency of the parental cells and optimal labeling conditions require experimental optimization. 


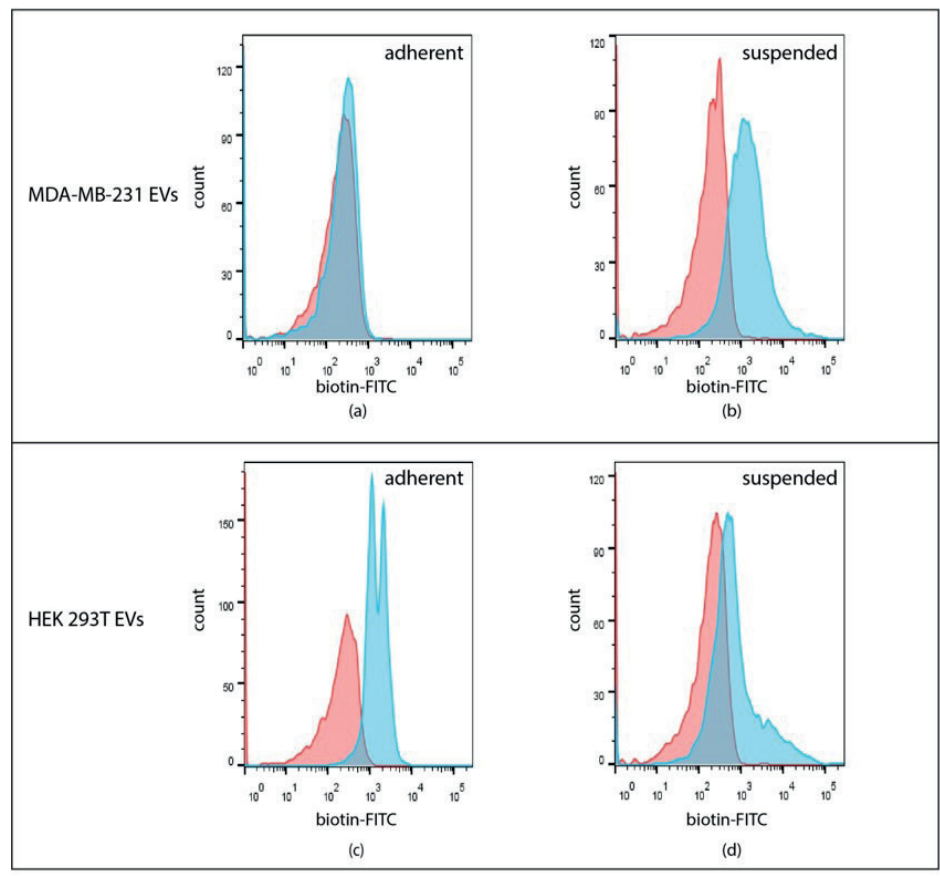

red $=$ EVs lipids (-)

blue $=$ EVs lipids $(+)$

FIGURE 2. Quantification of biotin on the surface of EVs shed from MDA-MB-231 $2 d$ cultured in adherent mode (a); suspended mode (b) and on the surface of EVs from HEK 293T cells cultured in adherent mode (c); suspended mode (d). EVs were pulled down by a CD63-dynabeads. EVs from cells cultured without DSPE-PEG-biotin are shown in red and with DSPE-PEG-biotin in blue.

\section{Salmonella bacteria surface cannot be modified by post-insertion strategy}

As another type of natural nanomedicine, bacteria-derived EVs trigger great interest for vaccination applications. Compared to their mammalian counterparts, bacteriaderived EVs appear easier to be scaled-up. In addition, the endogenous pathogenassociated molecular patterns (PAMPs) are efficient triggers for an immune response. The first FDA-approved product Bexsero ${ }^{\circledR}$ is a Neisseria meningitidis-derived OMVbased product used for protection against meningococcal infections. (Carter 2013) Within such context, an engineered Salmonella enterica serovar typhimurium SL3261 strain with hypervesiculation properties (, genes encodes Tol-pal proteins which link peptidoglycan layer and outer membrane layer) and low-toxicity (which encodes transferase important for LPS biosynthesis ) was selected in this study as it represents an interesting platform which can be used to display a range of antigens. (DalekeSchermerhorn, Felix et al. 2014) The post-insertion strategy may be an attractive approach to decorate the surface of the OMVs.

Bacteria were cultured in medium containing $40 \mathrm{~g} / \mathrm{mL}$ DSPE-PEG-Biotin and $5 \mathrm{~g} / \mathrm{mL}$ DSPE-PEG-FA for 3 days, after which membrane modification was assessed by FACS 


\section{Chapter 6}

and immuno-Electron Microscopy (EM). No labeling was detected on FACS (data not shown) and also no DSPE-PEG-biotin nor DSPE-PEG-FA modification was observed on the membrane surface of bacteria when ultra-thin section slides of the bacteria were examined with highly sensitive immune EM (Figure $3 a$ and $3 b$ ). We did observe FA-associated gold particles in the bacteria's cytoplasm (Figure 3b), however, these likely reflect endogenous FA storage.(Bermingham and Derrick 2002)

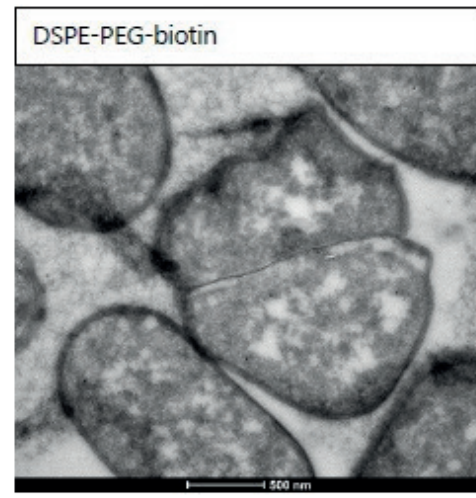

(a)

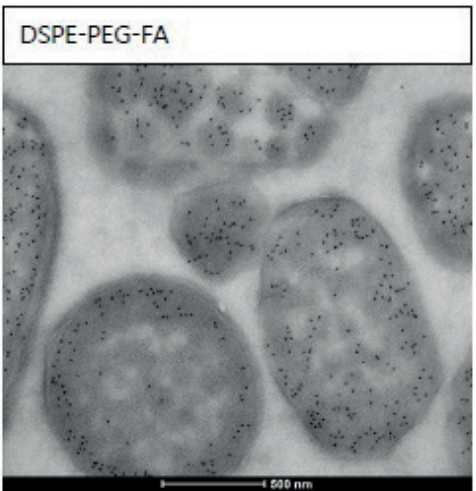

(b)

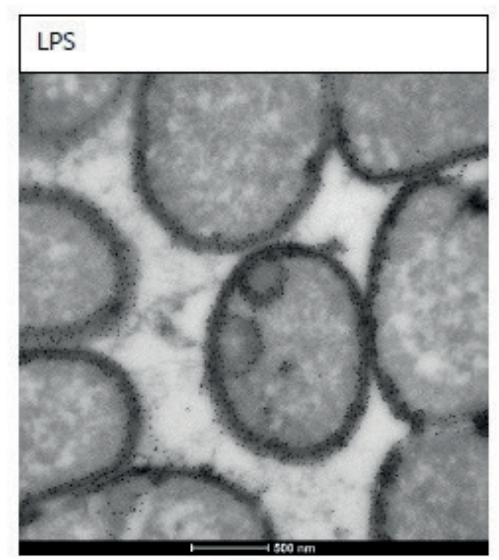

(c)

FIGURE 3. Immunogold labelling of bacteria Ultra-thin section slides. (a) No anti-biotin-collidal gold labeling is observed for DSPEG-PEG-biotin incubated bacteria nor is (b) anti-FA-colloidal gold membrane labeling observed for bacteria incubated with DSPE-PEG-FA. Intracellular FA staining is seen (c). As a positive control, extensive membrane labeling of anti-LPS-colloidal gold was observed.

As a positive control, LPS gold-labelling was observed on the surface of bacteria (Figure 3c). Together, these data suggest that lipids DSPE-PEG-biotin/ DSPE-PEG-FA were not inserted into membranes of bacteria. 
Salmonella-derived OMVs can be modified by post-insertion strategy independent of bacteria cells

TTo check the post-insertion efficiency on the OMVs released by those bacteria. Bacterial cells were removed by centrifugation and filtration, and OMVs were isolated from the filtrate by ultracentrifugation. A similar magnetic bead pulldown experiment as shown above for the mammalian EVs was performed on the isolated OMVs sample. Specifically, streptavidin-coated magnetic beads were used to capture DSPE-PEG-biotin positive OMVs. Subsequently, a one-step immunostaining with streptavidin-FITC was performed (Figure $4 \mathrm{~b}$ strategy 1 ). Flow cytometry results show that DSPE-PEG-biotin is indeed associated to the OMVs bound by the magnetic beads (Figure $5 a$ ). To rule out the possibility that biotinylated micelles were pulled-down, two two-step staining strategies were investigated. For these strategies (Figure $4 \mathrm{~b}$ strategy 2 and 3), we try to detect the other components present on the OMVs, thus FA moiety and LPS. We firstly check the presence of DSPE-PEG-FA which has similar structure of DSPE-PEG-biotin. Since the hypothesis for DSPE-PEGbiotin post-insertion mechanism is that its hydrophobic tail could insert onto the phospholipids bi-layer of OMVs, similar lipids should do the same trick. Next, if the OMVs were successfully capture by the beads, general OMV marker should be present. In our study, OMVs were captured using streptavidin-coated magnetic beads. Subsequently, the presence of FA was detected using an anti-folic acid antibody as primary antibody (Figure $4 \mathrm{~b}$ strategy 2); Alternatively, an anti-LPS antibody was used as the primary antibody (Figure $4 \mathrm{~b}$ strategy 3 ). The beads were subsequently counterstained with goat anti-mouse IgG APC conjugate and analyzed by flow cytometry. Both FA and LPS immunofluorescence were detected which indicates the presence of all three epitopes on the OMVs. (Figure 5a-b). Immuno-EM further confirmed this observation (Figure 6a-b). As an additional supporting evidence, after OMVs being enriched by the magnetic beads, lysis buffer was added to lyse the samples associated with the beads. A pronounced staining of outer membrane protein $\mathrm{C}(\mathrm{OmpC})$, a protein abundantly present on the membrane of gram-negative bacteria, was observed by immunoblot methods (Figure $6 \mathrm{c}$ ). Together these data indicate that although the bacterial cell themselves cannot be labeled using a postinsertion strategy, this strategy works for the OMVs derived from the bacteria and both DSPE-PEG-Biotin/DSPE-PEG-FA was assembled onto OMVs successfully. 


\section{Chapter 6}
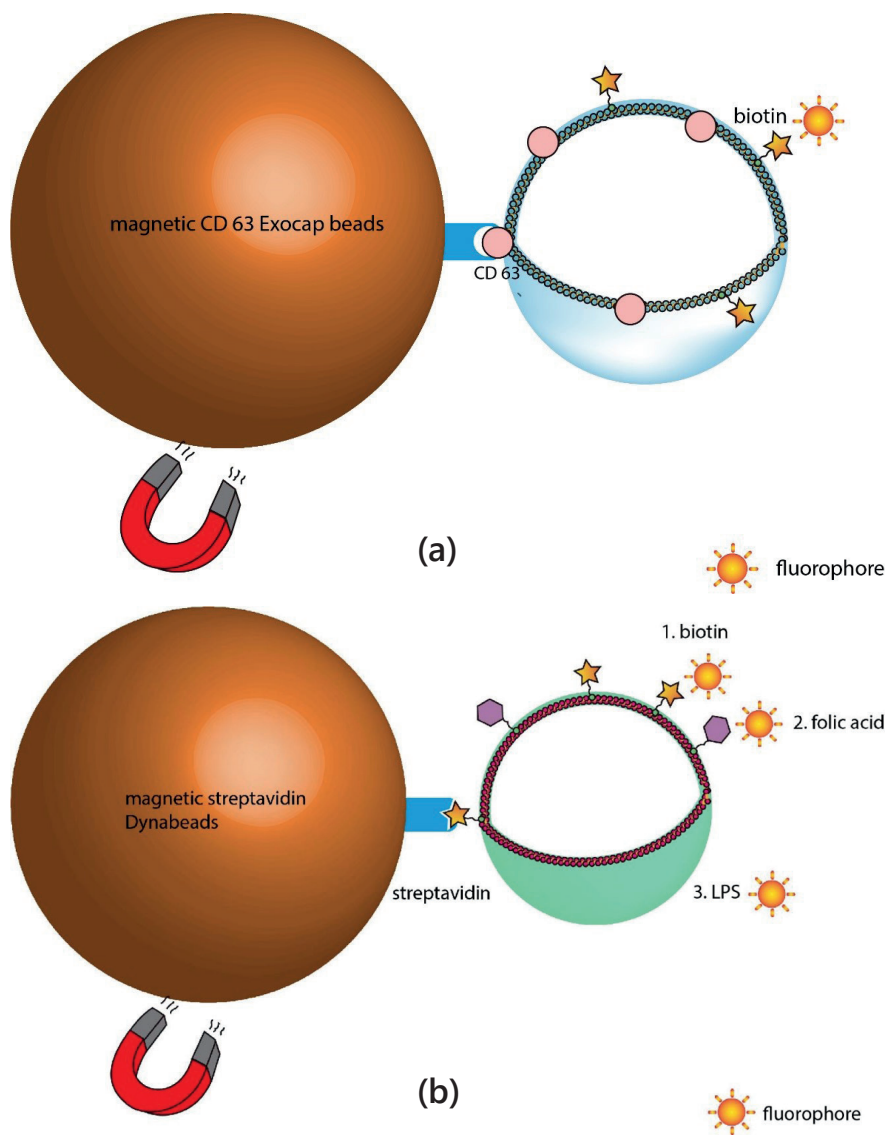

FIGURE 4. Bead pull-down strategies for mammalian cells-derived EVs (a) and bacteria-derived OMVs (b). Mammalian EVs are pulled by CD 63 Exocap beads firstly and then stained with streptavidin-FITC; Different detection strategies used to confirm the successful engineering of OMVs. OMVs were firstly pulled down by streptavidin magnetic beads, and the beads were 1) incubated with biotin-fluorophore conjugate; 2) incubated with mouse anti-folic acid antibody, and then incubate with rabbit anti-mouse fluorophore; 3 ) incubated with mouse anti-LPS antibody, and then incubate with goat anti-mouse IgG APC. 


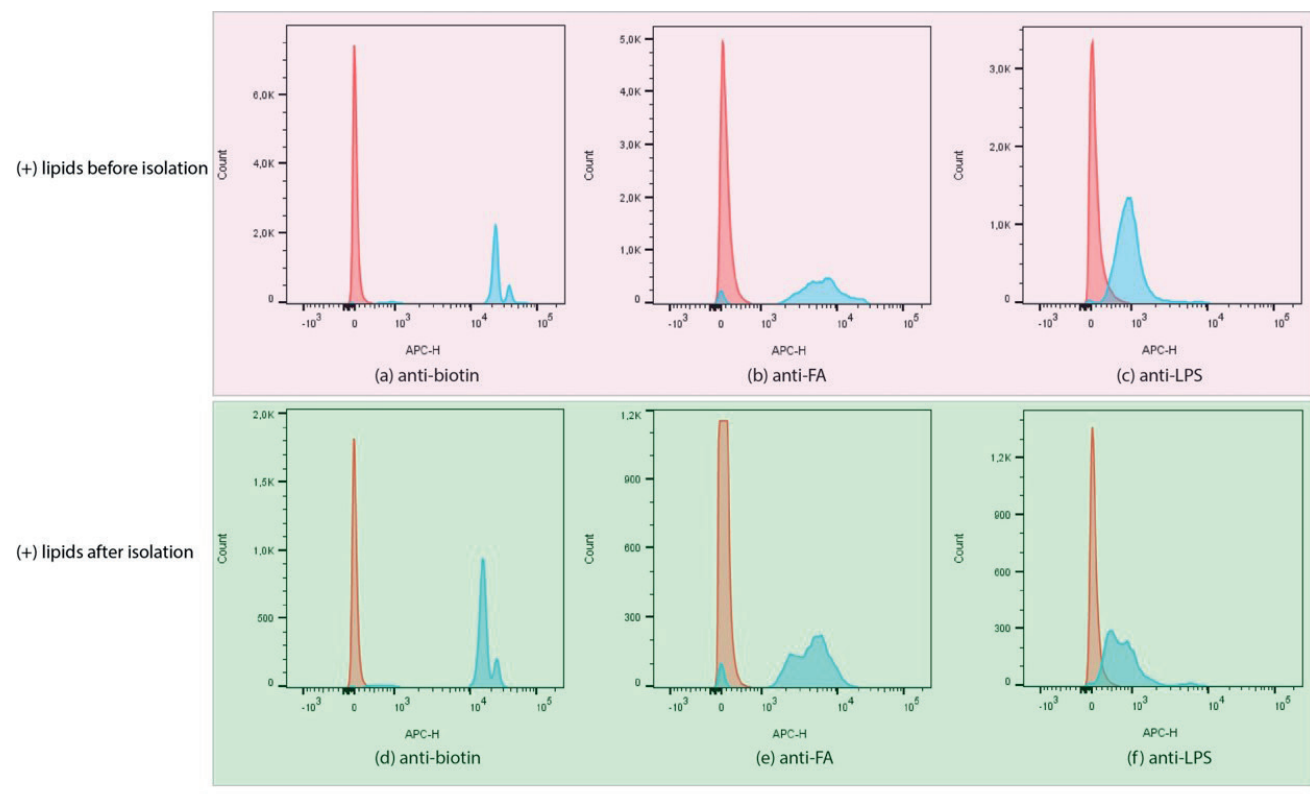

red $=$ EVs lipids $(-)$ blue $=$ EVs lipids $(+)$

FIGURE 5. Quantification of the DSPE-PEG-biotin, DSPE-PEG-FA, LPS on the surface of compositions pulled down by the streptavidin-magnetic beads when lipids were added during the bacteria culture (a)(c) and when lipids were added to the OMVs-containing medium only (d)-(f).

To further investigate whether OMV post-insertion is independent of the presence of the parental cells, bacteria were removed and the OMV-containing medium was filtered with 0.45 um filters. After this, the filtrate was incubated with DSPE-PEG-biotin/ FA. Interestingly, similar labeling efficiencies were observed as in the presence of the bacteria (Figure $5 d-f$ ), which suggests the post-insertion process occurs independent of the parental cells from which the OMV were derived. 


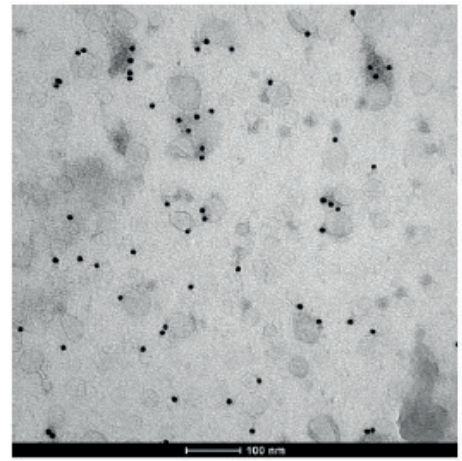

(a)

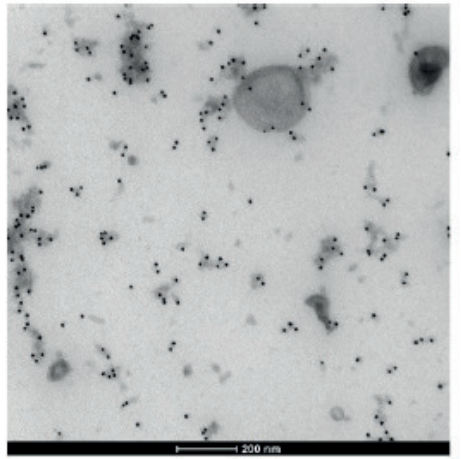

(b)

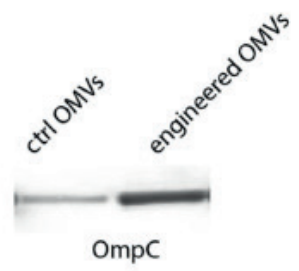

(c)

FIGURE 6. Immuno-gold labelling of DSPE-PEG-biotin (a) and DSPE-PEG-FA (b), PBS was used as an internal negative control in this experiment; OmpC protein immuno-blot of control OMVs and engineered OMVs (c).

\section{Conclusion \& discussion}

n summary, our data show that both mammalian EVs and bacteria-derived EVs can be readily modified by a post-insertion strategy. In addition, we demonstrate that the labelling efficiency can be improved by culturing mammalian cells in suspension mode. We also discovered differences between the modification process between mammalian-derived $\mathrm{EV}$ s and bacteria-derived $\mathrm{EV}$ s.

In the mammalian system, lipids first assemble into the donor cell membrane, and, as a result, EVs shed from those cells will also be modified. At present it is unclear how the ratio between exosomal labeling and microvesicle labeling is for this post-insertion strategy. It can be anticipated that the microvesicle labeling is more straightforward as it is not dependent on internalization and sorting.

As for bacteria, no insertion was detectable onto the surface of bacterial cells. This may be due to the fact that the outer membrane contains heavily glycosylated lipids which may have sterically shielded the surface of the cell. In contrast, lipids were found to more readily insert into the membrane of the highly curvature vesicles 
directly. This may be due to a different lipid composition of the OMVs compared to the membrane form which they originate or the physical characteristics of the OMVs. As OMVs represent a high curvature structure these often have lipid packing defects that may facilitate insertion.(Kastelowitz and Yin 2014) Taken together we propose different post-insertion mechanisms between mammalian EVs and bacterial OMVs. For mammalian EVs, cells incorporate the exogenous lipids into the cell membrane. The EVs shed from those modified cells are subsequently modified as well. As for bacteria, the exogenous lipids inserted into OMVs directly independent of the parental cells (Figure 7).

This lipids post-insertion strategy offers an easy and rapid approach to modify the surface of both mammalian cells and bacteria-derived EVs when compared with traditional genetic engineering approach. However, the in vitro and in vivo function of these lipids-modified EV are not known yet, future study reveal this information would be very important.
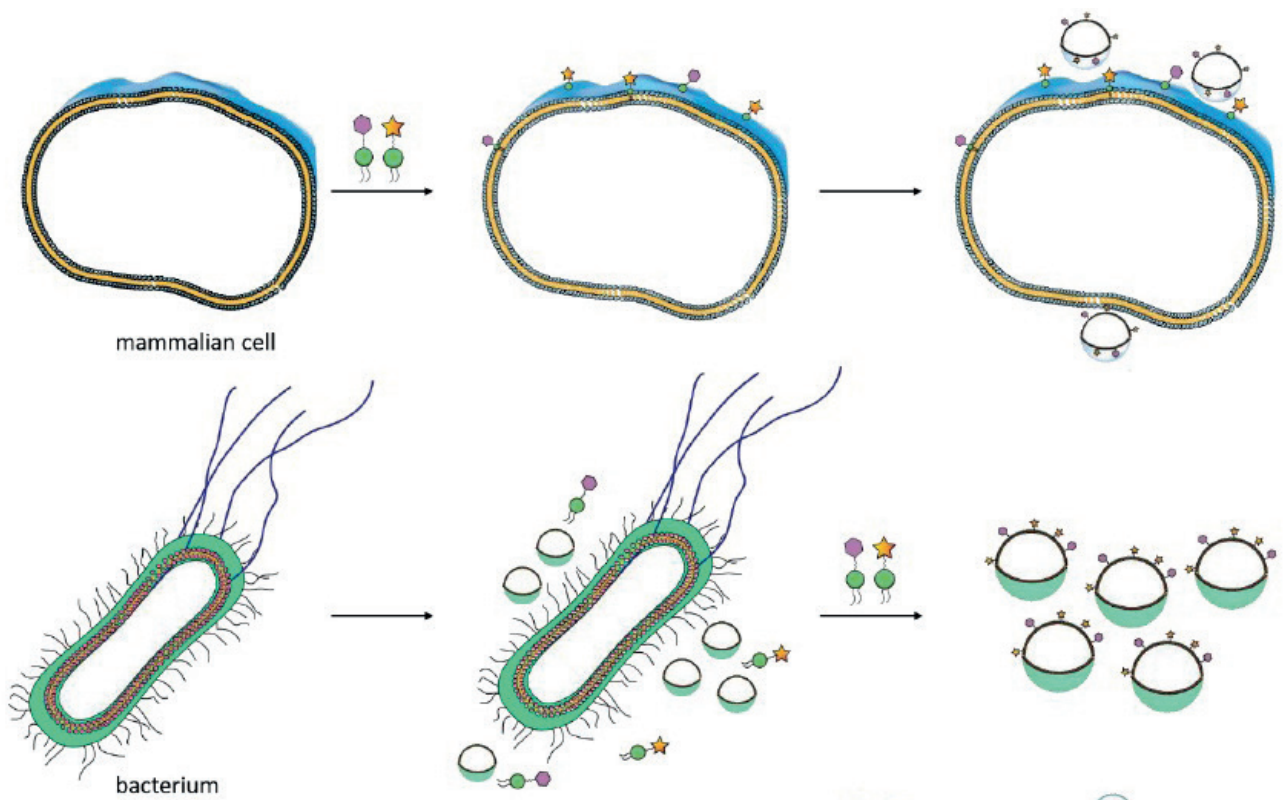

FIGURE 7. Lipids modification mechanism involved in mammalian-derived vesicles (upper row) and bacteria-derived vesicles (bottom row). 


\section{Chapter 6}

\section{Materials \& methods}

\section{Chemicals and reagents}

Mouse monoclonal anti-folic acid (F5766), Optiprep density gradient medium (D1556) and Halt Protease and Phosphatase Inhibitor Cocktail (ABCA3796199) were purchased from Sigma-Aldrich chemie. Anti-Salmonella typhimurium 0-4 antibody [1E6](AB8247) was purchased from Abcam. DSPE-PEG-2K-biotin (880129) was purchased from Avanti Polar Lipids, Inc. DSPE-PEG-2K-folate were purchased from Nanosoft polymers. Polyclonal Goat anti Mouse IgG APC (405308) was purchased from Biolegend. RIPA buffer (20-188) was purchased from Merck Millipore. Alexa Fluor 647-conjugated streptavidin was purchased from Jackson Immuno Research. And rabbit anti-Omp C antibody is in-house from Department of Medical Microbiolgy, UMC Utrecht. Dulbecco's modified eagle medium (DMEM, 42430-025), OptiMEM medium (51985) and Free-Style 293 medium (12338) was purchased from Thermofisher Scientific.

\section{Mammalian cell culture}

For adherent mode cell culture experiment, MDA-MB-231/HEK 293T cells were seeded at the density of $2 \times 10^{5}$ in $2 \mathrm{~mL}$ medium (DMEM supplemented with $10 \%$ fetal bovine serum and $1 \%$ Penicillin-Streptomycin) in 6-well plate, and cultured with/without the addition of DSPE-PEG-biotin $(5 \mathrm{~g} / \mathrm{mL})$, and cultured in a normal incubator maintained at $37^{\circ} \mathrm{C}$ and $5 \% \mathrm{CO}_{2}$.

For suspended mode cell culture experiment, MDA-MB-231/HEK 293T cells were seeded at the density of $2 \times 10^{5}$ in $2 \mathrm{~mL}$ medium, and culture in a shaker embedded inside the incubator.

Cells were collected and stained for $15 \mathrm{~min}$ at room temperature with StreptavidinAlex488 conjugate (final conc. $1 \mathrm{ug} / \mathrm{mL}$ in $1 \%$ FBS-containing PBS) and analyzed by BD FACS Canto II ${ }^{\mathrm{TM}}$. SPHERO ${ }^{\mathrm{TM}}$ Rainbow Calibration Particles (Spherotech) are used to determine the voltages for the uniform calibration of the flow cytometers.

\section{Mammalian cells-derived EVs isolation and bead assay}

For EVs isolation, cells were cultured in adherent and suspended mode as mentioned before, and washed and continued culturing with OptiMEM ${ }^{\mathrm{TM}}$ for 2 days. Remove the cells by serial centrifugation steps, firstly $300 \mathrm{~g}$ for 5 mins and then $2000 \mathrm{~g}$ for 15 mins at room temperature. The EVs in the supernatant was captured by CD63 ExoCap beads (JSR life sciences) overnight at 4, and the beads were subsequent counterstained with Streptavidin Alexa-488 and analyzed by BD FACSCanto ${ }^{\mathrm{TM}}$. 


\section{Preparation of LB-0 medium}

$10 \mathrm{~g}$ bacto tryptone (Oxoid LP0042) and $5 \mathrm{~g}$ yeast extract (Oxoid LP0021) were added in $1 \mathrm{~L} \mathrm{milliQ}$ water and autoclaved at 121 for $15 \mathrm{~min}$.

\section{Bacteria culture and $\mathrm{OMVs}$ isolation}

Streaked bacteria from glycerol stock and inoculated in $2.5 \mathrm{~mL}$ LB-0 medium ( $2 \mathrm{mM}$ $\mathrm{MgCl}_{2^{\prime}}, 2 \mathrm{mM} \mathrm{CaCl}{ }_{2}$ LB-0, $0.2 \%$ glucose, $25 \mu \mathrm{g} / \mathrm{ml}$ kanamycin) at 30 overnight, and scale up of the overnight culture in fresh LB medium (subculture ratio 1:40) with the addition of DSPE-PEG-biotin $(40 \mu \mathrm{g} / \mathrm{mL})$ and DSPE-PEG-FA $(5 \mu \mathrm{g} / \mathrm{mL})$ for 3 days.

Bacteria were removed by centrifuging the culture medium at $2000 \mathrm{~g}$ for $10 \mathrm{~min}$ at 4. And the supernatant was filtered through $0.45 \mathrm{~m}$ filter with syringe. The filtered medium was then subject to ultracentrifugation at $48000 \mathrm{rpm}$ at 4 for $1 \mathrm{~h}$ and $15 \mathrm{mins}$ (50.2Ti rotor, Beckman). The crude pellet were resuspened in $60 \%$ iodixanol solutions and then subject to gradient ultracentrifugation $(60 \%, 55 \% 50 \%, 45 \%, 40 \%, 35 \%$, $30 \%, 25 \%, 20 \%, 15 \%, 10 \%, 5 \%$ and $0 \%$ ) (SW40Ti rotor, Beckman) at $39000 \mathrm{rpm}$ at 4 for $14 \mathrm{~h}$. 3 fractions $(15 \%, 20 \%, 25 \%)$ were pooled and concentrated by $100 \mathrm{KD}$ centrifugal filter (Amicon). The concentrate was washed with PBS for 3 times to remove the excess iodixanol.

\section{Bacteria ultra-thin section}

Ultrathin bacteria slides are prepared with a diamond knife at -120 on a Leica UCT Ultracut (cryo sectioning). Bacteria section were picked up with looping device filled with mixture of methylcellulose and sucrose. Samples are incubated with freshly carbon coated formvar grid (Hexagonal $\mathrm{Cu}$ and processed following the Tokuyasu method. Firstly, samples are fixated in PBS solutions containing $2 \%$ paraformaldehyded and $0.2 \%$ glutaraldehyde for $1 \mathrm{~h}$ at room temperature. Then washed with PBS. Afterwards, samples are added to warm $12 \%$ gelatin solution and pelleted by centrifugation. Rapid cooling of the sample-containing gelatin are performed by placing it on ice, which results in the solidification of the gelatin. Pellet of fixated bacteria in solidified gelatin is retrieved from eppendorf vial and cut with a clean razor blade into small cubic blocks $\left(\sim 1 \mathrm{~mm}^{3}\right)$. Sucrose infiltration steps are performed by incubating the gelatin blocks in $2.3 \mathrm{M}$ sucrose PBS solution overnight in a carrousel.

Samples were incubated with freshly carbon-coated grids. Grids with samples were incubated on several droplets of 40 phosphate buffer. Then inactivate the fixative with $0.15 \%$ Glycine. Blocking step was performed with $1 \%$ BSA-containing PBS. Immuno- labeling were performed with the rabbit anti-biotin antibody (1:10000)/ mouse anti-folic acid antibody (1:200)/ mouse anti-LPS antibody (1:200), staining and embedding in Uranyl Oxalate/Uranyl Methylcellulose solution. Tecnai 20 Twain MK2 (5022/11) was used for acquiring images. 


\section{Chapter 6}

\section{OMVs immuno-gold labelling and TEM imaging}

For folic acid immuno-gold labelling, OMVs in PBS were adsorbed to carbon-coated formvar grids for 10 mins at room temperature, washed 3 times in PBS droplets, block in 1\% BSA for 10min and incubate with mouse anti-folic acid antibody (1st Antibody) for 20 min, wash 3 times in 1\% BSA and then incubate with rabbit anti-mouse antibody (1:250 dilution, bridging antibody from UMC Utrecht, the Netherlands) for 10mins. Intensive washing steps were performed (7 times in PBS and 1 time in 1\% BSA), and incubate in $10 \mathrm{~nm}$ protein A-gold for $15 \mathrm{mins}$ and fixated in $2 \%$ paraformaldehyde and $0.2 \%$ glutaraldehyde-containing PBS. After 8 times washing with milliQ water, grids were embedded in methyl cellulose uranyl-acetate $(\mathrm{pH} 4)$. For biotin immunogold labelling, similar steps as folic acid labelling were used, and rabbit anti-biotin antibody (1:10000 dilution, UMC Utrecht, the Netherlands) was used and the bridging step was omitted. Grids were imaged using a Tecnai T12 transmission electron microscope (FEl, Eindhoven, The Netherlands).

\section{Beads pull down and flow cytometry}

In a 96-well round-bottom plate, 0.2 I Dynabeads ${ }^{\mathrm{TM}}$ M-280 Streptavidin (11205D, Thermofisher) per well were incubated with samples for $2 \mathrm{~h}$ at room temperature while shaking. Using the magnetic plate to pull down the beads, and remove the sup ernatant. Beads were blocking in 1\% BSA, and afterwards incubate with 50ul diluted mouse anti-folic acid antibody (1:200 dilution)/ mouse anti-Samonella antibody (1:200) for $1 \mathrm{~h}$ at room temperature. After the removal of $1 \mathrm{st}$ antibody and washing, add Polyclonal Goat anti Mouse IgG APC (405308 Biolegend, 1:500 dilution) and incubate for $1.5 \mathrm{~h}$ at room temperature while shaking (keep plate in dark). Wash away the excess fluorophore and resuspend the beads in 200I 1\% BSA solution. Flow cytometry analysis was performed with FACSCanto II ${ }^{\mathrm{TM}}$ (BD science, USA). And for biotin analysis, Alexa Fluor 647-conjugated streptavidin (1:600) was incubated with beads for $1 \mathrm{~h}$ at room temperature.

\section{SDS-PAGE and Western blot}

To reach the maximum pull down efficiency of the OMVs in the sample, $0.5 \mathrm{I}$ beads per well were added, and after incubating with beads for $2 \mathrm{~h}$ at room temperature while shaking. The supernatant was removed, and beads were washed 3 times with PBS. And lyse the OMVs with 1x RIPA buffer supplemented with 1x protease inhibitor cocktail. Samples were mixed with reducing sample buffer containing dithiothreitol (DTT), heated to $95^{\circ} \mathrm{C}$ for 10 mins and subjected to electrophoresis over $4-12 \%$ BisTris polyacrylamide gels (Thermo Fisher Scientific). Gels were stained with PageBlue Protein Staining Solution (Thermo Fisher Scientific) according to manufacturer's instructions. Alternatively, proteins were blotted on Immobilon-FL polyvinylidene 
difluoride (PVDF) membranes (Merck Millipore, Amsterdam, The Netherlands), which were subsequently blocked with $50 \%$ v/v Odyssey Blocking Buffer (LI-COR Biosciences, Leusden, The Netherlands) in Tris buffered saline (TBS). Antibody incubations were performed in $50 \% \mathrm{v} / \mathrm{v}$ Odyssey Blocking Buffer in TBS containing $0.1 \% \mathrm{v} / \mathrm{v}$ Tween 20 (TBS-T). Primary antibody are in-house rabbit anti-OmpC antibody (dilution 1:50). And secondary antibody is Alexa Fluor 680-conjugated anti-rabbit antibodies (Thermo Fisher Scientific, A-21076, 1:10000). Imaging was performed on an Odyssey Infrared Imager (LI-COR Biosciences, Leusden, the Netherlands) at $700 \mathrm{~nm}$.

\section{Acknowledgement}

L.J. would like to thank PhD Candidate Dennis Doorduijn (Department of medical microbiology, UMC Utrecht, the Netherlands) for useful discussion.

\section{Conflict of Interest}

J.L. and W.S.P.J. are involved in Abera Bioscience $A B$, which aims to exploit outer membrane vesicle-based delivery technologies. 


\section{Chapter 6}

\section{References}

Allen, T. M., et al. (2002). "Use of the post-insertion method for the formation of ligand-coupled liposomes." Cellular and Molecular Biology Letters 7(2): 217-219.

Bermingham, A. and J. P. Derrick (2002). "The folic acid biosynthesis pathway in bacteria: evaluation of potential for antibacterial drug discovery." Bioessays 24(7): 637-648.

Carter, N. J. (2013). "Multicomponent meningococcal serogroup B vaccine $\left(4 C M e n B ;\right.$ Bexsero $\left.{ }^{\circledR}\right)$ : a review of its use in primary and booster vaccination." BioDrugs 27(3): 263-274.

Chuo, S. T.-Y., et al. (2018). "Imaging extracellular vesicles: current and emerging methods." Journal of biomedical science 25(1): 1-10.

Daleke-Schermerhorn, M. H., et al. (2014). "Decoration of outer membrane vesicles with multiple antigens by using an autotransporter approach." Appl. Environ. Microbiol. 80(18): 5854-5865.

Fais, S., et al. (2016). "Evidence-based clinical use of nanoscale extracellular vesicles in nanomedicine." ACS nano 10(4): 3886-3899.

Jiang, L., et al. (2019). "Bacterial membrane vesicles as promising vaccine candidates." European Journal of Pharmaceutics and Biopharmaceutics.

Kastelowitz, N. and H. Yin (2014). "Exosomes and microvesicles: identification and targeting by particle size and lipid chemical probes." Chembiochem 15(7): 923-928.

Kooijmans, S., et al. (2016). "PEGylated and targeted extracellular vesicles display enhanced cell specificity and circulation time." Journal of controlled release 224: 77-85.

Nguyen, M. M., et al. (2015). "Enzyme-Responsive Nanoparticles for Targeted Accumulation and Prolonged Retention in Heart Tissue after Myocardial Infarction." Advanced materials 27(37): 5547-5552.

Torchilin, V. (2011). "Tumor delivery of macromolecular drugs based on the EPR effect." Advanced drug delivery reviews 63(3): 131-135.

Zhang, W., et al. (2017). "Magnetic and folate functionalization enables rapid isolation and enhanced tumor-targeting of cell-derived microvesicles." 11(1): 277-290.

Zhu, L., et al. (2017). "Folate-engineered microvesicles for enhanced target and synergistic therapy toward breast cancer." 9(6): 5100-5108.

Zitvogel, L., et al. (1998). "Eradication of established murine tumors using a novel cell-free vaccine: dendritic cell derived exosomes." Nature medicine 4(5): 594-600. 


\section{Supplementary Information}

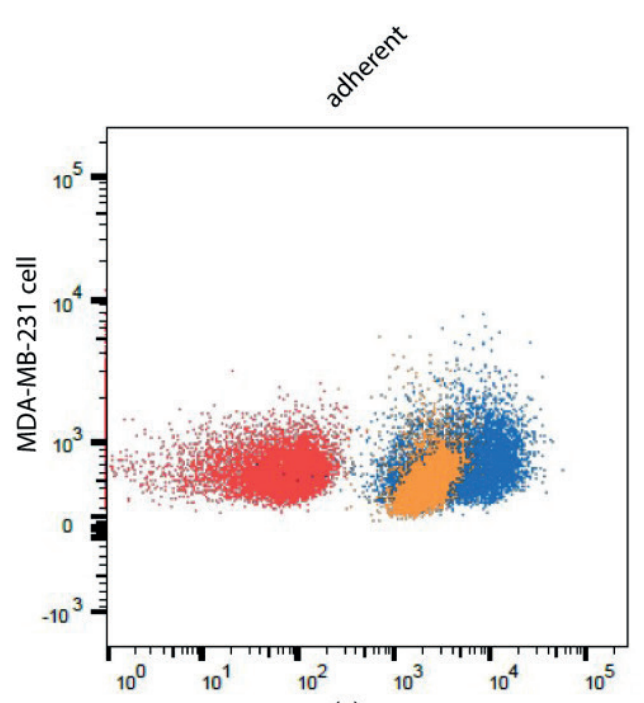

(a)

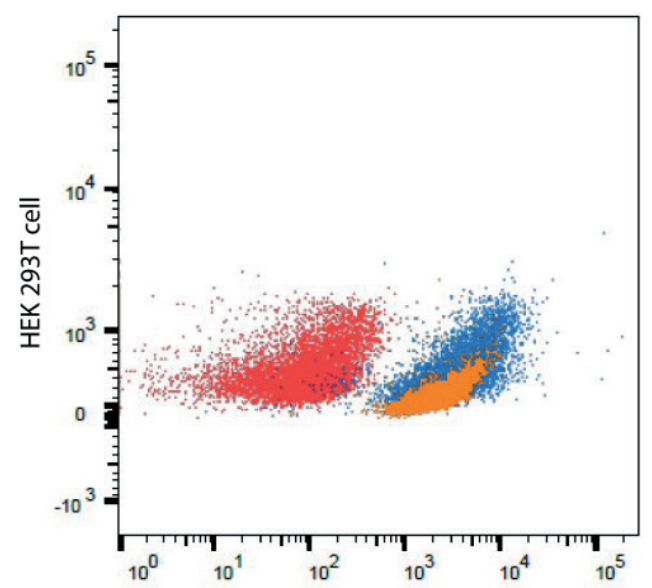

(c)

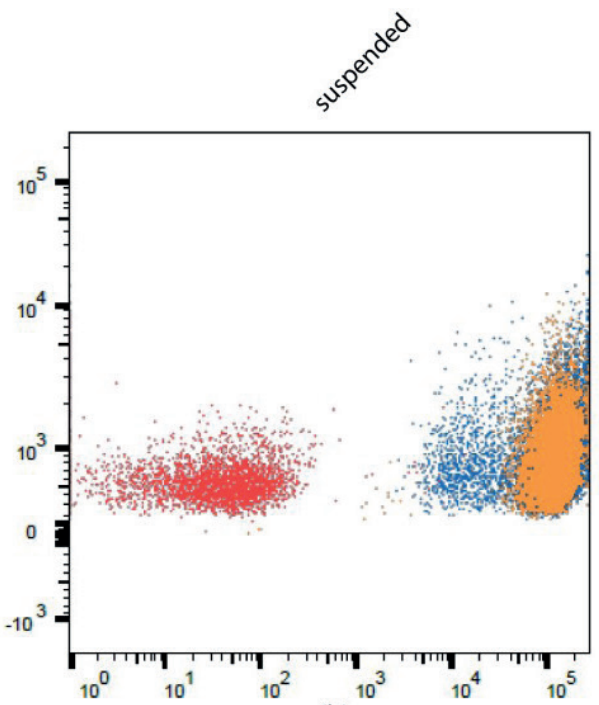

(b)

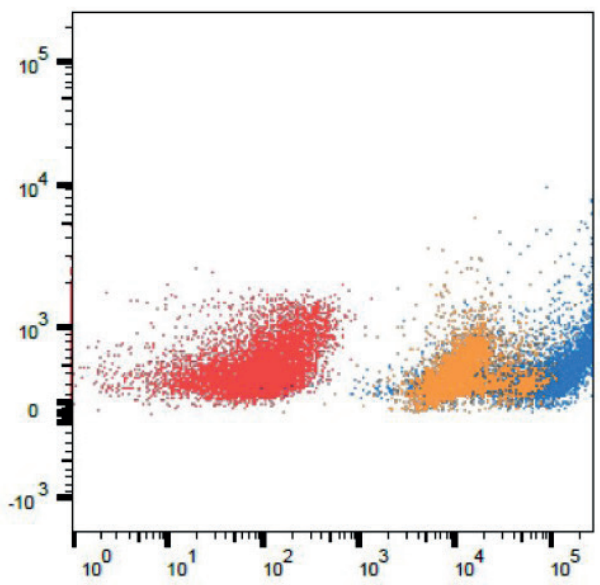

(d)

$(-)$ DSPE-PEG-biotin

(+) DSPE-PEG-biotin culture for 2d

(+) DSPE-PEG-biotin culture for 5d

FIGURE S1. Quantification of biotin on the surface of MDA-MB-231 cells after $2 d$ and $5 d$ culture in adherent mode (a); suspended mode (b) and on the surface of HEK 293T cell in adherent mode (c); suspended mode $(d)$. 


\title{
Chapter 7
}

\section{Immunomodulatory effects of Salmonella typhimurium OMVs in an intestine-mimicking co-culture system- a preliminary study}

\author{
Linglei Jiang ${ }^{1 *}$, Veronica Ayechu Muruzabal ${ }^{2 *}$, Max van \\ Essen $^{1 *}$, Linette E. M. Willemsen ${ }^{1}$, Pieter Vader ${ }^{1}$, Johan \\ Garssen $^{2,3}$, Raymond Schiffelers'
}

1. Department of clinical chemistry and Haematology, UMC Utrecht, the Netherlands

2. Division of Pharmacology, Utrecht Institute for Pharmaceutical Sciences, Faculty of Science, Utrecht University, Utrecht, The Netherlands

3. Department of Immunology, Nutricia Research, Utrecht, The Netherlands

4. Department of Cardiology, UMC Utrecht, the Netherlands

* These authors contributed equally.

Corresponding author: R.Schiffelers@umcutrecht.nl 


\section{Chapter 7}

\section{Abstract}

Salmonella infection can cause serious disease symptoms such as diarrhea, fever, abdominal cramps, and vomiting. It is usually acquired by consuming unhygienic food and it is of rising concern globally. Currently, effective and safe vaccines against Salmonella are lacking. Outer membrane vesicles (OMVs) which are nanosized, phospholipid bilayered membrane bound structures can be released by Gramnegative bacteria and play important roles in bacteria-bacteria as well as bacteriahost interactions. The Pathogen-Associated Molecular Patterns (PAMPs) that are displayed on OMVs can trigger innate immunity responses, and OMVs can be considered safer when compared with live-attenuated bacteria vaccines.(Jiang, Schinkel et al. 2019) Hence, Salmonella-derived OMVs could serve as a promising vaccine candidate for combating salmonellosis.

Oral delivery of vaccines is considered as safe and easy-to-execute when compared with other administration routes such as intravenous, intramuscular and intradermal injection. In this study, an in-vitro intestine-mimicking platform was set up to evaluate the immunogenicity of OMVs from a detoxified, hypervesiculating Salmonella strain msbB, tolRA SL3261. Our study shows that Salmonella-derived OMVs can effectively modulate the host response by activating the intestinal epithelial cells.

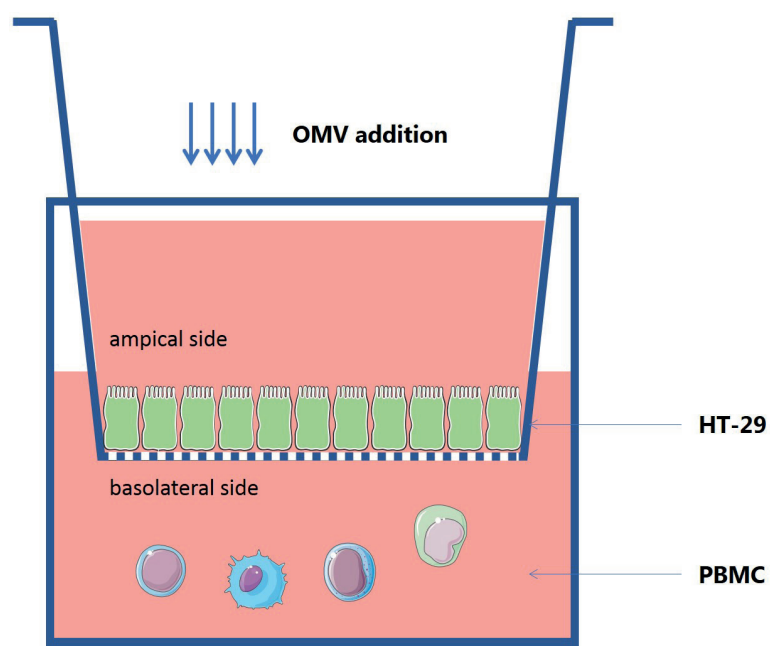




\section{Introduction}

Salmonella bacteria can be genetically engineered to use them as vaccines (Kang, Srinivasan et al. 2002) However, the use of live bacteria has potential safety concerns as they can replicate, which may lead to infections or strong immune reactions. This provides a rationale for new vaccination approaches that are both effective and safe.

Extracellular vesicles (EVs) are nanosized structures composed of phospholipid bilayers, which can be secreted by all domains of life: eukaryotes, bacteria, and archaea. EVs from Gram negative bacteria are termed 'Outer membrane vesicles $(\mathrm{OMVs})^{\prime}$. OMVs play important roles in communication between bacteria as well as between bacteria and host, and are considered important for bacterial survival and shaping the host's immune reaction. (Jan 2017)

OMVs have several attractive properties for use as vaccine candidates. They display pathogen-associated molecule patterns on their surface, which can trigger innate and adaptive immune responses in the host. These may be further enhanced by proinflammatory molecules in the OMVs interior. In addition, OMVs do not replicate and therefore present a safer alternative to a vaccine composed of live attenuated bacteria. An attractive opportunity is the fact that bacteria can be readily engineered to enhance beneficial traits or remove or downregulate adverse qualities

In this study, a genetically engineered msbB tolRA Salmonella enterica serovar Typhimurium (S. typhimurium) strain SL3261 was used as OMVs donor. By knocking out the gene $m s b B$, which encodes acyltransferase, hexa-acylated lipopolysaccharide (LPS) can no longer be synthesized and the toxicity of the OMVs will be reduced. By deleting $\triangle$ tolRA, the donor bacteria become hypervesiculating and as a result higher yields of OMVs can be obtained.

To study the opportunities for these OMVs as oral vaccination vehicles, an intestinemimicking co-culture system was established. Specifically, a human colon cancer cell line HT29 was cultured on a transwell system. It forms a monolayer which mimics the human intestinal environment, and adopts a polarized morphology under the specific culture condition we have used. This system has already been used fully in the study of drug/food absorption, transport and release by epithelial cells.(Martínez-Maqueda, Miralles et al. 2015, Hayen, Otten et al. 2018). Here we evaluated the interaction of OMVs through the HT29 intestinal epithelial cell (IEC) and evaluated the response of the epithelial monolayer as well as basolateral PBMCs on OMV exposure. 


\section{Chapter 7}

\section{Results}

\section{SL326 1 OMV size distribution and morphology}

OMVs were isolated by ultracentrifugation. The average size of isolated OMVs was $0.1+/-0.0 \mu \mathrm{m}$ as determined by nanoparticle tracking analysis (NTA) (Figure 1a). Transmission electronic microscopy (TEM) showed classic cup-shaped images of isolated OMVs., They appear heterogeneous in size, morphology, and electron density (Figure 1b). This may indicate different formation processes and may represent different subclasses of OMVs. These differences could be investigated further by different isolation methods or a refined density gradient ultracentrifugation protocol.

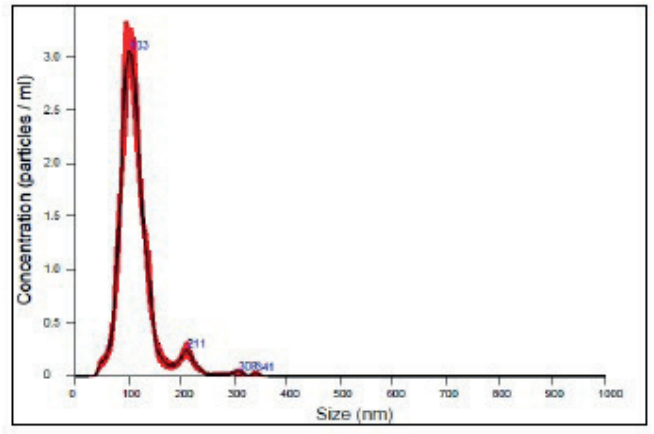

(a)

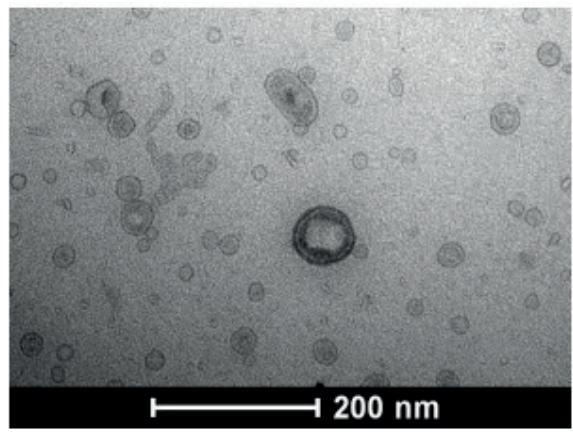

(b)

FIGURE 1. a) Size distribution of SL3261-derived OMVs measured by NTA; b) Transmission electron microscopy image of SL3261 OMVs.

\section{Uptake of OMVs by HT29 cells and Peripheral Blood Mononuclear Cells (PBMCs)}

OMVs were fluorescently labelled with the lipidic dye PKH67. When HT29 cells were incubated with labelled OMVs, pronounced uptake by cells was seen. No signal was detected when PBMCs were incubated with labelled OMVs (Figure 2). This was somewhat unexpected as the PBMC preparation contains many cell types that are responsible for the uptake of pathogens. Since OMVs contain many of the same surface molecules as their parental cell it was assumed that this would result in uptake.

To quantitatively evaluate the uptake by these cells, we analyzed samples by flow cytometry. Uptake of OMVs by HT29 cells upon incubation with PKH67-labelled OMVs was observed (Figure $3 b$ ), which is consistent with the uptake phenomena 
observed by microscopy (Figure 2). Interestingly, the complete population of cells demonstrated this shift. This peak shift was not apparent in HT29 cells incubated with a 10-fold lower dose of OMVs (Figure 3b). PBMCs did not show uptake at either dose (Figure 3d).

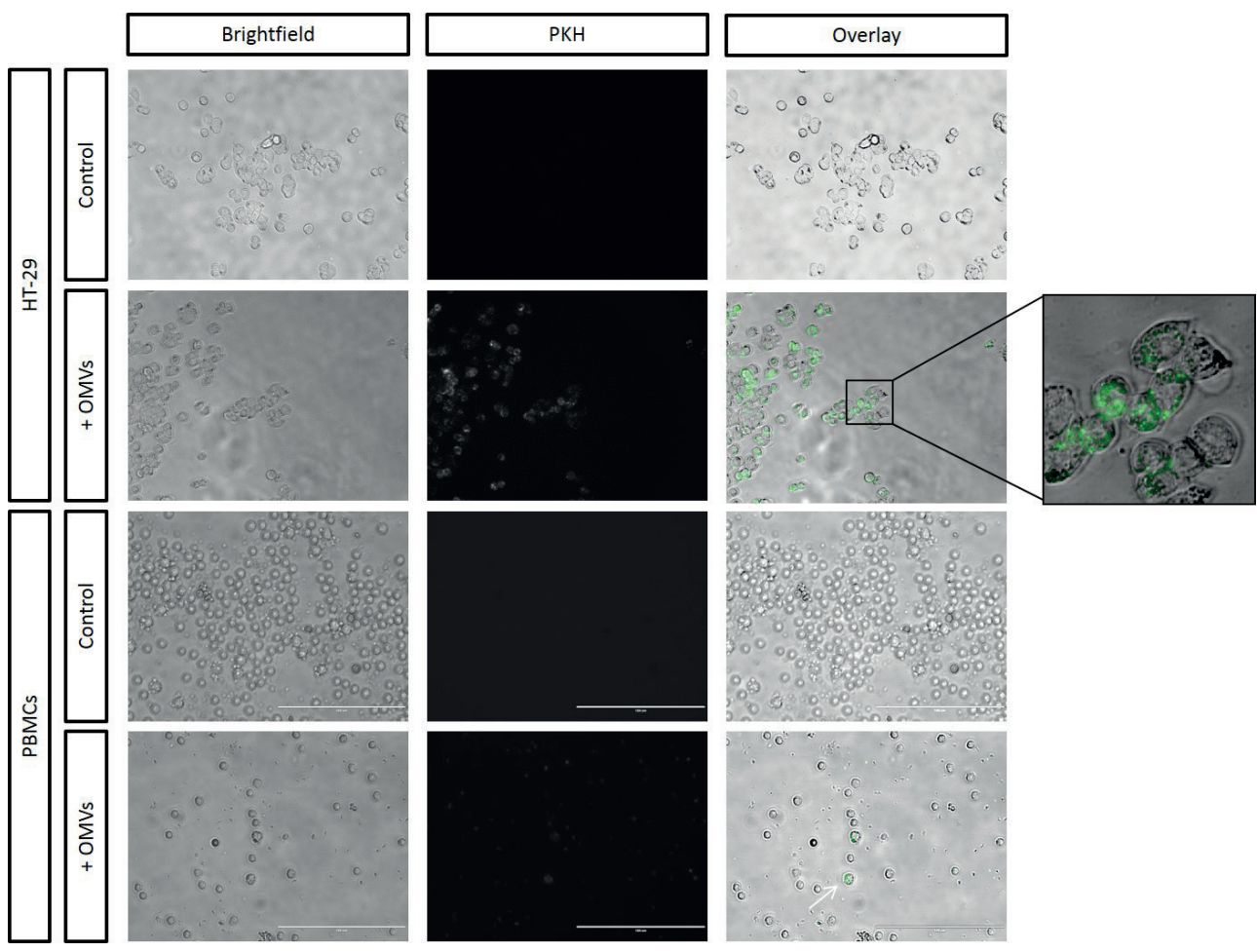

FIGURE 2. Brightfield and fluorescent microscope images. HT29 cells and PBMCs incubated with fluorescent labelled or unlabelled OMVs. PKH: a green fluorescent dye with long aliphatic tails (PKH67) which can incorporate into the lipid region of OMVs.

\section{Cytokine profile released by PBMCs and HT29/PBMC co-culture}

To evaluate the consequences of the interaction with cells, different doses of OMVs were added to the apical side of the transwell co-culture system containing PBMCs in the acceptor compartment. Culture medium from this compartment was collected after 24 hours exposure. OMVs did not induce significant cytotoxicity both in the HT29/PBMC co-culture or when directly incubated with PBMCs (supplementary Figure S1).

Apical addition of OMVs did induce a significantly increased secretion of proinflammatory cytokines, i.e. Interferon- (IFN-), TNF- $\alpha$, IL-6, IL-8, and IL-10 in both HT29/PBMC co-culture and PBMC monoculture ( $p<0.05)$, but not IL-13. (Figure 4) 


\section{Chapter 7}

However, when the HT29 intestinal epithelial cell (IEC) layer was present, the levels of IFN-, TNF- $\alpha$ and IL-10 were less markedly increased than when IEC was not present. Surprisingly, for IL-6 and IL-8, released levels are similar when IEC layer is present or not. Also, even when IEC layer was present, OMV samples are able to induce more IFN- $\gamma$ and TNF- $\alpha$ secretion than LPS, and these levels are even higher compared to direct exposure of PBMCs. When IEC layer was absent, OMVs induced a significant increased IFN- $\gamma$ and TNF- $\alpha$ production in a dose-dependent manner $(p<0.05)$.

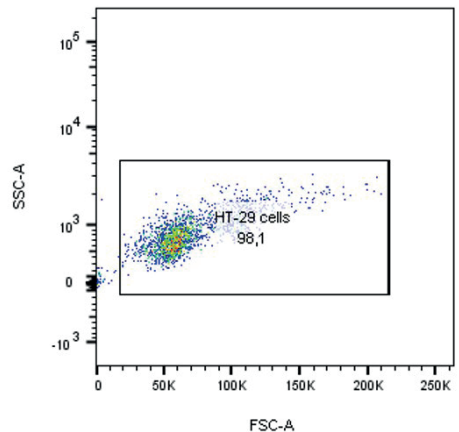

(a)

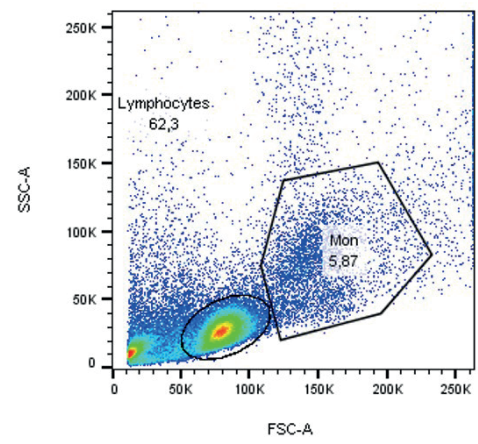

(c)

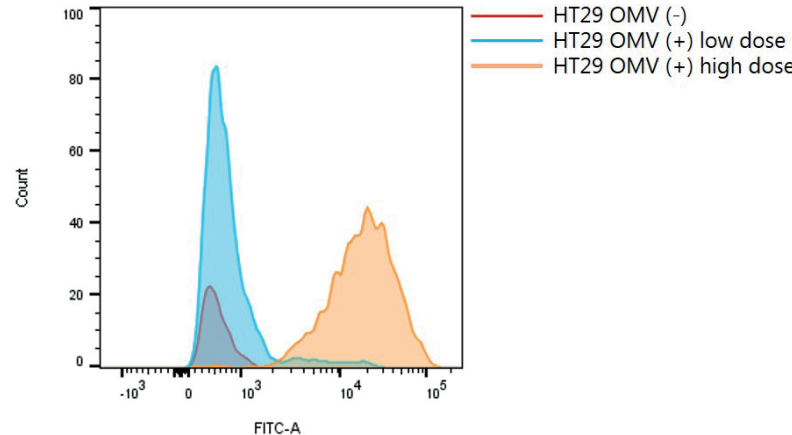

(b)

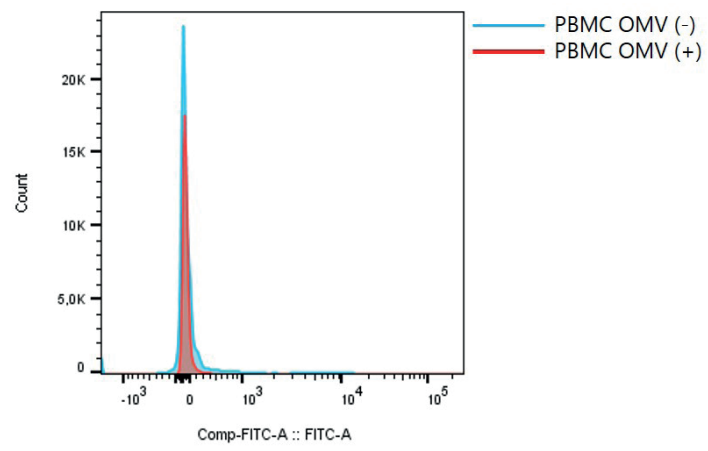

(d)

FIGURE 3. Dot plot of HT29 cell after incubated with fluorescently labelled OMVs (a) and PBMCs when incubated with fluorescently labelled OMVs (c); histogram of HT29 cell after incubated with fluorescently labelled OMVs (b) and PBMCs when incubated with fluorescently labelled OMVs (d).

These results indicate that OMVs are able to mount immune responses from intestinal cell/PBMCs in a co-culture system. This may indicate that this system can be explored to investigate the immune stimulation profile form engineered OMVs for vaccine purposes. It may also be an attractive set-up to test the effect of microbiomederived OMVs on intestinal cells and their communication to systemic circulation. Refinements of the current set-up could include a long-term cytokine expression profile, and to study the effects of indirect exposure. 


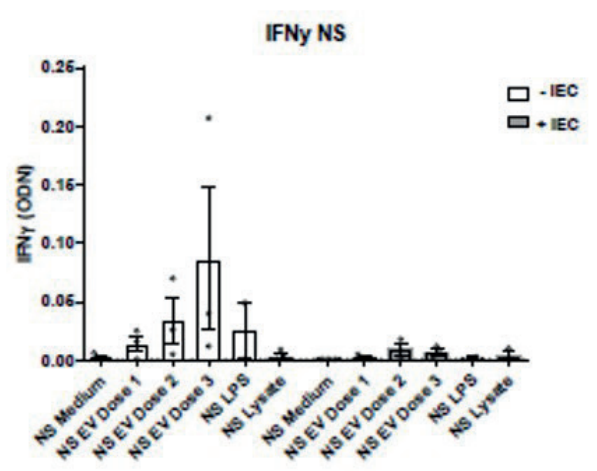

(a)

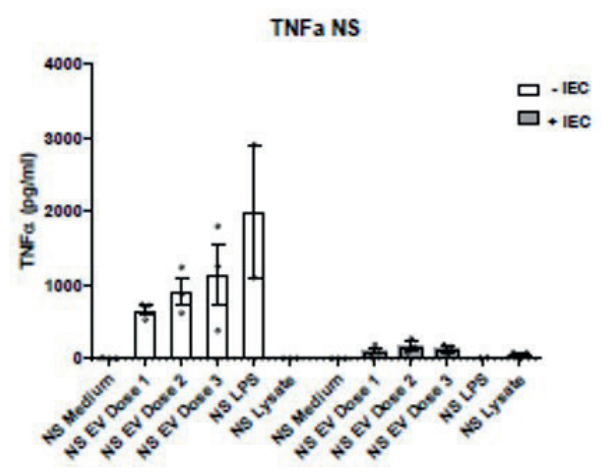

(c)

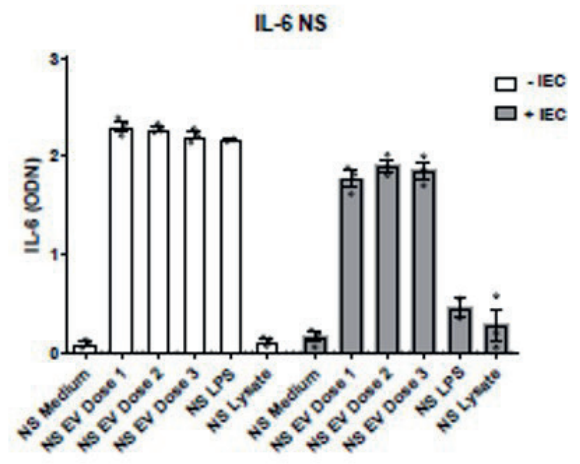

(e)

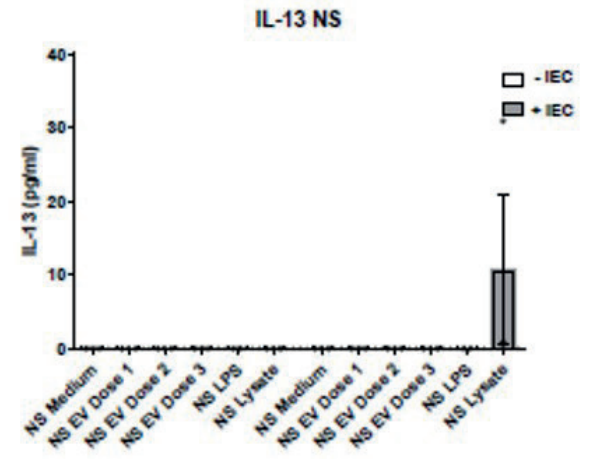

(b)

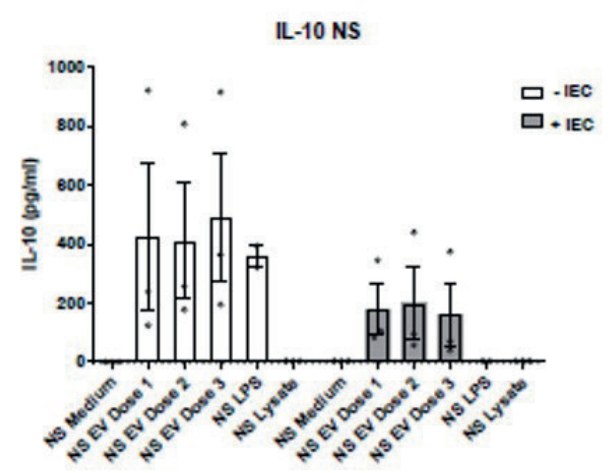

(d)

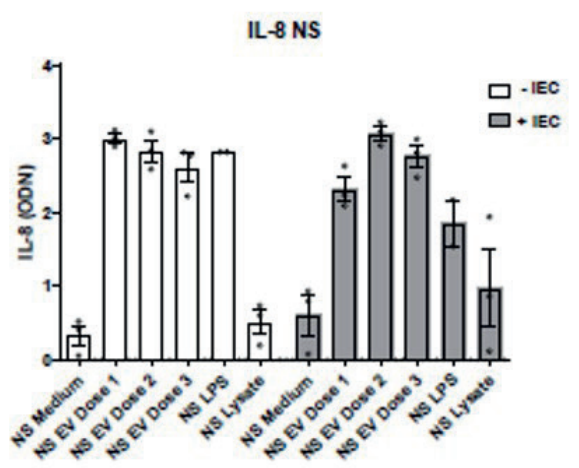

(f)

FIGURE 4. Cytokine levels measured by ELISA in direct stimulation of PBMCs and HT29/PBMC co-culture condition. 


\section{Chapter 7}

\section{Materials \& methods}

\section{Bacterial culture and $\mathrm{OMV}$ isolation}

The hypervesiculating and LPS deficient S. typhimurium strain SL3261 was cultured in Luria-Bertani broth with $0.2 \%$ glucose and $25 \mathrm{ug} / \mathrm{mL}$ Kanamycin at $30^{\circ} \mathrm{C}$. Bacterial cultures were scaled up twice and at an Optical Density (OD) ${ }_{600}$ of 1.2, bacteria were pelleted at $4000 \mathrm{xg}$. The OMV containing supernatant was collected and filtered through a 0.45um filter (Sartorius Stedim Biotech, Göttingen, Germany). OMVs were subsequently pelleted by ultracentrifugation at $208.000 \mathrm{xg}$ for $75 \mathrm{~min}$ (Beckman Coulter Type 50.2 Ti Rotor). Pellets were then washed once with $0.5 \mathrm{M} \mathrm{NaCl}$ and pelleted again at $208.000 \mathrm{xg}$ for $75 \mathrm{~min}$. After washing, OMV pellets were resuspended in medium of the final destination, and if required snap-frozen in liquid nitrogen and stored at $-80^{\circ} \mathrm{C}$ until further use. Total protein amount in each batch was measured by Bicinchoninic acid (BCA) assay in order to create reproducibility and equal amounts of OMVs were used in each experiment.

\section{Size distribution and transmission electron microscopy}

OMV size and concentration were measured using nanotracking particle analysis (NTA) (Nanosight NS500, Malvern Panalytical) and analysed with NTA 3.3 Development Build software (Malvern Instruments Ltd.). OMV samples for transmission electron microscopy (TEM) imaging were collected before snap-freezing and incubated with freshly carbon coated electron microscopy grids. Grids were then washed with distilled water and blocked with $0.1 \%$ BSA in PBS. OMVs were then fixed on the grids with $0.2 \%$ glutardialdehyde/2\% paraformaldehyde. Finally, after another washing step with distilled water, grids were embedded in Uranyl methylcellulose $\mathrm{pH}$ 4.0. Grids were imaged using Tecnai-12 Electron microscope.

\section{HT29 culture and PBMC isolation}

A transwell ${ }^{\circledR}$ system(Corning Life Sciences, Corning, NY, USA) with $0.4 \mu \mathrm{m}$ pore size was used in order to mimic the human gut in vitro. The human colon carcinoma cell line HT29 (ATCC, HTB-38) was used as intestinal epithelial cell layer. Once HT29 cells formed a complete monolayer, PBMCs were added to the lower well. HT29 cells were cultured in T-75 flasks (Greiner Bio-One) in McCoy's 5A medium (modified) (Gibco, Thermo Fisher Scientific), supplemented with 5\% foetal bovine serum (FBS) and $0.5 \%$ penicillin/streptomycin. The cells were passed 1:20 each week and medium was changed every 3 days. After reaching sufficient confluency, HT29 cells were seeded on to the upper well of the transwell ${ }^{\circledR}$ systems and cultured for another week until confluent. Light microscopy was used for visual assessment of confluency. In the transwell ${ }^{\circledR}$ system, medium was changed every 2 days. 
Peripheral blood mononuclear cells (PBMCs) were isolated from whole blood. Whole blood was diluted 2 times in Dulbecco's Phosphate Buffered Saline (PBS) (Sigma-Aldrich). The whole blood suspension was then added to a $50 \mathrm{~mL}$ tube with $15 \mathrm{~mL}$ Ficoll-Paque and tubes were centrifuged in swinging buckets at $400 \mathrm{xg}$ for 30 minutes at room temperature (Hettich Zentrifugen, ROTINA 380R, Germany). The mononuclear cell layer was collected and cells were counted using Flow cytometry. During all experiments, $2 \times 10^{6}$ cells were added to each recipient well. When PBMCs were added to the transwell ${ }^{\circledR}$ system, medium was changed to RPMI (Gibco, Thermo Fisher Scientific) with $2.5 \%$ FBS and $1 \%$ penicillin/streptomycin in all wells to reduce influences of the culture medium on cytokine production.

\section{Experimental conditions and cytokines}

In the cytokine study, the concentration of OMVs is calculated by microBCA (Thermo Fisher). Stimulations were performed with OMVs (dose 1: $50 \mathrm{mg} / \mathrm{mL}$; dose 2: 150mg/ $\mathrm{mL}$; dose $3500 \mathrm{mg} / \mathrm{mL}$ ) or bacterial lysates $(100 \mathrm{ng} / \mathrm{ml})$ from SL3261 strains.

Alongside with OMVs, OMV lysates, $1 \mathrm{ug} / \mathrm{mL}$ LPS and cell culture medium served as controls in all experiments. Levels of IFN- $\gamma$, TNF- $\alpha$, IL-6, IL-8, IL-12p70 (Invitrogen, Vienna, Austria), and IL-10(U-Cytech Biosciences, Utrecht, The Netherlands) in the bottom wells were measured by sandwich Enzyme-Linked Immuno Sorbent Assay (ELISA). Differences between different conditions were assessed using one-way ANOVA and p-values below 0.05 were considered significant.

\section{OMV uptake by HT29 and PBMCs}

Uptake of OMVs by both HT29 cells and PBMCs was assessed by addition of fluorescently labelled OMVs to each cell type in monoculture. OMVs were isolated as described previously and labelled using PKH67GL (Sigma-Aldrich), following the manufacturer's protocol. Two different dosages of PKH67 labelled OMVs were used to assess dose dependent uptake. The low and high dose of OMVs contained $3.97 \times 10^{10}$ and $3.97 \times 10^{11}$ particles respectively. HT29 cells were seeded at the density of $2.5 \times 10^{6}$ cells/well in a 24 -well plate 1 day before OMVs were added. Unlabelled SL3261 OMVs were added to control wells. Upon exposure to the OMVs, wells were incubated for 4 hours at $37 \mathrm{C}$. Afterwards, fluorescence was directly visualized in the culture plate using EVOS Cell Imaging Systems (Life Technologies, Grand Island, NY, USA). Following visualization, cells were trypsinized with $1 \%$ trypsin and fixed with 4\% paraformaldehyde. Fluorescein (FITC) intensity of HT29 cells and PBMCs was measured with flow cytometry (BD FACS canto ii, BD Biosciences) for quantitative measurement. 


\section{Chapter 7}

\section{Cytotoxicity of OMVs}

The possible cytotoxic effects of OMVs on PBMCs and HT29 cells were investigated by measurement of lactate dehydrogenase $(\mathrm{LDH})$ release with a Lactate Dehydrogenase Activity Assay (Sigma Alderich, St. Louis, USA). LDH concentrations were measured in supernatants of the HT29/PBMC co-cultures that were incubated for 24 hours with different concentrations of OMVs as well as the earlier mentioned controls.

\section{Discussion}

This study investigates the immune modulatory effects of OMVs produced by $S$. typhimurium. A clear increase of pro-inflammatory cytokine levels was detected in a co-culture system mimicking the human intestinal environment in vitro. OMVs were able to elicit an immune response across a barrier of intestinal epithelial cells. While direct stimulation of PBMCs with SL3261 OMVs induced the greatest immune response, intestinal epithelial cells could internalize OMVs and indirectly stimulate PBMC in the recipient cells. This suggests that the epithelial cell layer serves as a barrier of the passage of OMVs, but also mediates bacteria host communication. Cytokine levels did not differ significantly between intact OMVs and lysed OMVs. This can be due to the fact that the immune response is triggered by a certain composition of OMVs, which do not necessarily have to be intact. Long-term production of TNF$\alpha$ was seen after exposure to OMVs, whereas IL-6, IL-8 and IL-10 nearly normalized after $48 \mathrm{~h}$.

An earlier study has also demonstrated S. typhimurium OMV induced production of TNF- $\alpha$ by dendritic cells.(Alaniz, Deatherage et al. 2007) This phenomena is in accordance with the observation in this study.

The production of TNF- $\alpha$ by macrophages is normally instantaneous upon stimulation in vivo, so the release of TNF- $\alpha$ may be overlooked. (Van Deventer, Buller et al. 1990, Parameswaran and Patial 2010) The secretion of TNF- $\alpha$ interplays with other signalling pathways and cytokines, for example, it can be upregulated by Toll-like receptor 4 (TLR-4) induced NF-KB activation and downregulated by IL-10. (Dickensheets, Freeman et al. 1997, Anderson 2000) Another cytokine that supress the secretion of TNF- $\alpha$ is IL-6. (Schindler, Mancilla et al. 1990)

Interleukin-6 is a cytokine with pro- and anti-inflammatory properties, and is produced by both macrophages and intestinal epithelial cells. IL- 6 production can be seen as a protective mechanism that limits tissue injury caused by toxins and infectants.(Papanicolaou, Wilder et al. 1998) Although a no clear production by HT29 cells was observed, OMVs induced significantly elevated IL-6 levels in both PBMC monocultures and HT29/PBMC co-cultures. This suggests that OMVs are able to 
activate the pathways involved in IL-6 production in a similar fashion as their parent bacteria.(Klimpel, Asuncion et al. 1995) This may indicate that OMV can plays similar role as the inactivated Salmonella vaccine.

It has long been known that interferon- $\gamma$ plays a crucial role in the immune response against Salmonella infections.(Bao, Beagley et al. 2000) IFN- $\gamma$ is essential to eliminate Salmonella bacteria from invaded macrophages.(Ingram, Brodsky et al. 2017) Levels of IFN- $\gamma$ tend to rise over the course of days, and measuring production 24 hours after exposure to OMVs might be too early. Because IFN- $\gamma$ production from 24- to 48 hours after OMV exposure also did not show increased production, even this timeframe might be too short. The possibility that OMVs are not able to induce the same inflammatory response as their parent Salmonella bacterium can also not be excluded.

The most interesting finding is the successful elevation anti-inflammatory cytokine IL-10. IL-10 inhibits the development of $\mathrm{T}$ cell differentiation into Th1 cells and cytotoxic T cells, and dampens NK cell responses.(Salazar, Peñaloza et al. 2017) IL-10 can inhibits a lot of pro-inflammatory marker such as IL-6, TNF- $\alpha$ and IFN- $\gamma$ and serve as a therapeutic agent. (Fioranelli and Roccia 2014) This study shows that SL3261 OMVs are able to induce IL-10 production in the HT29/PBMC co-culture, much like their donor bacteria. This again highlights the immune modulatory properties of Salmonella-derived OMVs can potentially be used as a vaccine platform.

Research on OMVs as new vaccine candidates has drastically increased in the past years. In 2013, a N. meningitidis OMV vaccine has been approved by the European Medicines Agency. OMV vaccines promise a better immunogenic capacity than subunit vaccines due to the close resemblance of the parent bacteria. In contrast to inactivated live vaccines, OMVs might also induce the same fast immune response as live vaccines, but with increased safety because of the inability to replicate. OMVs also offer the possibility of modification and engineering of these vesicles. Refinements of (membrane-)protein composition can further potentiate the immune response or even provide immunity against other bacterium species.

Besides, OMVs from S. typhimurium could even induce cross-protection against other Salmonella species, expanding the potential use of OMV vaccines.(Liu, Liu et al. 2016) The present study suggests that OMVs can induce an inflammatory response that is similar, but not identical to the response to their donor Salmonella bacteria. It can be used as a safer oral vaccine agent. In addition, this intestinal-mimicking coculture system can also be a good alternative to in vivo platforms for evaluation of oral vaccine. 


\section{Chapter 7}

\section{Reference}

Alaniz, R. C., et al. (2007). "Membrane vesicles are immunogenic facsimiles of Salmonella typhimurium that potently activate dendritic cells, prime $B$ and $T$ cell responses, and stimulate protective immunity in vivo." The Journal of Immunology 179(11): 7692-7701.

Anderson, K. V. (2000). "Toll signaling pathways in the innate immune response." Current opinion in immunology 12(1): 13-19.

Bao, S., et al. (2000). "Interferon- $\gamma$ plays a critical role in intestinal immunity against Salmonella typhimurium infection." Immunology 99(3): 464-472.

Dickensheets, H. L., et al. (1997). "Interleukin-10 upregulates tumor necrosis factor receptor type-II (p75) gene expression in endotoxin-stimulated human monocytes." Blood, The Journal of the American Society of Hematology 90(10): 4162-4171.

Fioranelli, M. and M. G. Roccia (2014). "Twenty-five years of studies and trials for the therapeutic application of IL-10 immunomodulating properties. From high doses administration to low dose medicine new paradigm." J Integr Cardiol 1(1): 2-6.

Hayen, S. M., et al. (2018). "Exposure of Intestinal Epithelial Cells to Short-and Long-Chain FructoOligosaccharides and CpG Oligodeoxynucleotides Enhances Peanut-Specific T Helper 1 Polarization." Frontiers in immunology 9.

Ingram, J. P., et al. (2017). "Interferon- $\gamma$ in Salmonella pathogenesis: New tricks for an old dog." Cytokine 98: 27-32.

Jan, A. T. (2017). "Outer membrane vesicles (OMVs) of gram-negative bacteria: a perspective update." Frontiers in microbiology 8: 1053 .

Jiang, L., et al. (2019). "Bacterial membrane vesicles as promising vaccine candidates." European Journal of Pharmaceutics and Biopharmaceutics.

Kang, H. Y., et al. (2002). "Immune responses to recombinant pneumococcal PspA antigen delivered by live attenuated Salmonella enterica serovar Typhimurium vaccine." Infection and immunity 70(4): 1739-1749.

Klimpel, G. R., et al. (1995). "Cholera toxin and Salmonella typhimurium induce different cytokine profiles in the gastrointestinal tract." Infection and immunity 63(3): 1134-1137.

Liu, Q., et al. (2016). "Outer membrane vesicles from flagellin-deficient Salmonella enterica serovar Typhimurium induce cross-reactive immunity and provide cross-protection against heterologous Salmonella challenge." Scientific reports 6: 34776.

Martínez-Maqueda, D., et al. (2015). HT29 cell line. The Impact of Food Bioactives on Health, Springer, Cham: $113-124$.

Papanicolaou, D. A., et al. (1998). "The pathophysiologic roles of interleukin-6 in human disease." Annals of internal medicine 128(2): 127-137.

Parameswaran, N. and S. Patial (2010). "Tumor necrosis factor- $\alpha$ signaling in macrophages." Critical Reviews $^{\mathrm{TM}}$ in Eukaryotic Gene Expression 20(2).

Salazar, G. A., et al. (2017). "Interleukin-10 production by T and B cells is a key factor to promote systemic Salmonella enterica serovar typhimurium infection in mice." Frontiers in immunology 8: 889.

Schindler, R., et al. (1990). "Correlations and interactions in the production of interleukin-6 (IL-6), IL-1, and tumor necrosis factor (TNF) in human blood mononuclear cells: IL-6 suppresses IL-1 and TNF."

Van Deventer, S., et al. (1990). "Experimental endotoxemia in humans: analysis of cytokine release and coagulation, fibrinolytic, and complement pathways." 


\section{Supplementary Information}

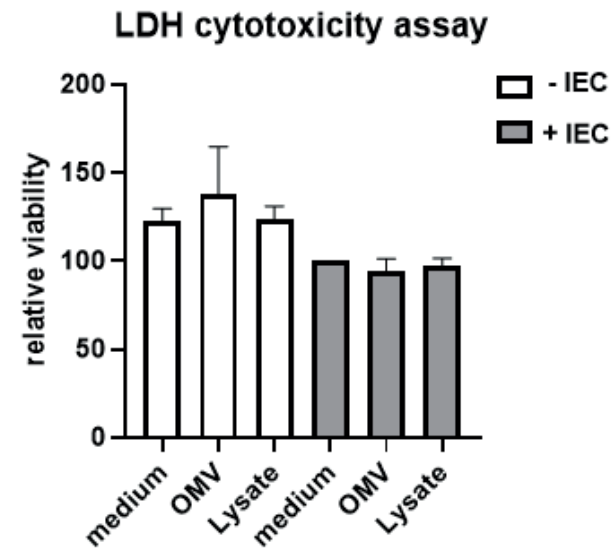

FIGURE S1. Cell viability results calculated by LDH cytotoxicity assay. 


\section{Chapter 8}

Summary 


\section{Chapter 8}

Nanomedicine is the application of nanotechnology in disease diagnosis and treatment. Nanomedicines can be obtained by either chemical synthesis or from natural sources. Investments into development of synthetic nanomedicine have yielded different drug delivery systems such as micelles, nanogels, dendrimers and liposomes. Naturally occurring and endogenous nanomedicine systems include for example extracellular vesicles that are secreted by living cells. Both synthetic and natural nanomedicine systems hold enormous promise as drug delivery systems, as they may allow 1) targeted delivery of therapeutic agents to the site of disease; 2) improve solubility of hydrophobic drugs; 3) co-delivery of two or more drug molecules simultaneously thus allowing combinational therapy; 4) increase the half-life of drug molecules in the circulation by protecting drug molecules from degradation and rapid renal elimination.(Farokhzad and Langer 2009, Choi and Han 2018)

Over the past decades, with joint efforts from both academia and industry, a substantial number of nanomedicine-based products have become available for clinical application. In addition, there is an increasing interest in nucleic acids-based drugs in recent years. However, existing drug delivery systems are mainly suitable for delivery of small molecular drugs, while delivery of novel emerging drugs such as high molecular-weight and highly charged nucleic acid drugs remains a challenge. (Wahlich, Desai et al. 2019)

In chapter 2, the characterization of a poly(amido amine)-based nanogel was reported. Cre mRNA was loaded into these nanogels. In vitro data showed that uncoated nanogels were superior in transfecting cells compared to their poly(ethylene glycol)coated counterparts. In addition, transfection of Cre-recombinase mRNA was tested in Ai9 Cre-recombinase reporter mice after subcutaneous injection. In contrast to in vitro observations, here the poly(ethylene glycol) coated nanogels were superior in the number of cells that were transfected in the subcutaneous injection area, followed by the non-coated nanogels, followed by free mRNA which was essentially devoid of activity. Leakage of mRNA expression to other tissues was essentially absent demonstrating the highly localized activity of nanogel-mediated delivery.

In chapter 3, we discussed the possibility of using a novel nanomedicine platform, i.e. EVs, as carriers for nucleic acids. As EVs can functionally transfer biological cargoes such as DNA/RNA, and proteins to the recipient cells, they are very likely to facilitate the transfer of exogenous therapeutic nucleic acids as well. Their versatile molecular compositions make manipulation of their content and properties feasible. In short, EVs are an efficient and safe alternative for nucleic acids drug delivery system.

Besides the promises EVs hold as drug carrier, bacteria-derived EVs, also bear potential as vaccine candidates against bacterial infections. Firstly, pathogen associated molecular patterns on the surface of bacteria-derived EVs are able to 
illicit both innate and adaptive immune responses. Secondly, bacteria-derived EVs are non-replicate which is considered to be safe. In chapter 4, we mainly discussed the isolation procedures and characterization methods for bacterial EVs, engineering approaches to enable precise tailoring of membrane vesicles (MVs) to achieve safe, highly immunogenic and efficient vaccines are also included. The successful development story of Bexsero, a commercially available EV-based vaccine product against Neisseria meningococcus infection, is also being reviewed.

As EVs from Gram-positive bacteria are less well studied compared with their counterparts from Gram-negative bacteria, the escape of EVs escape from the thick cell walls of Gram-positive bacteria remains an unsolved question, and knowledge regarding the immunogenicity of these EVs is also lacking. Therefore, in chapter 5, Gram-positive bacteria Enterococcus faecium E1162 and its isogenic mutants were chosen as the study subject. We investigated the morphology, size, yield, protein compositions and immunogenicity of EVs released from the nosocomial pathogen Gram-positive bacterium E. faecium wild-type (WT) strain E1162 and two E1162 isogenic mutants yajC and AltA. Different EVs subpopulations were observed among EVs from E1162 and its mutants. Qualitative and quantitative proteomics of EVs revealed that there is a selective protein sorting mechanism during EV biogenesis. Furthermore, analysis showed that both E1162 WT EV and E1162 DyajC EV contained higher abundance of potential immunogenic proteins such as Pbp5, LysM, AdcAfm 2410 and AdcAfm 2344 when compared with E1162 $\triangle$ AltA. Finally, it was shown that both $\mathrm{E} 1162 \mathrm{EVs}$ and $\triangle y a j C \mathrm{EVs}$ illicited cytokine release and long-term immune response in a murine model, which suggests that $E$. faecium-derived EVs are promising vaccine candidates against $E$. faecium infection.

To improve the targeting and immunogenic properties of EVs from bacteria, a simple post-insertion approach was investigated in chapter 6. DSPE-PEG-biotin lipids can be incorporated into the phospholipid bilayer membranes of EVs from both mammalian cells and bacteria. The introduction of biotin in the EV membrane opens the possibility of EV decoration with streptavidin-antigen conjugates. We also discovered the differences between the modification process between mammalianderived EVs and bacteria-derived EVs. In mammalian systems, lipids first assemble into the donor cell membrane, as a result, EVs shed from those cells will also be modified. As for bacteria, no insertion of DSPE-PEG lipids was detectable on the surface of bacterial cells but high signal can be detected on the surface of bacterial EVs.

Finally, a co-culture system was set up to evaluate the immunogenicity of outer membrane vesicles (OMVs) in chapter 7 with the aim to develop an in-vitro platform for the oral vaccination possibility evaluation of OMVs. These results indicate that 


\section{Chapter 8}

OMVs are able to mount immune responses from intestinal cells/ peripheral blood mononuclear cells (PBMCs) in a co-culture system. This may indicate that this system can be explored to investigate the immune stimulation profile form engineered OMVs for vaccine purposes. It may also be an attractive set-up to test the effect of microbiome-derived OMVs on intestinal cells and their communication to systemic circulation. Refinements of the current set-up could include a long-term cytokine expression profile, and to study the effects of indirect exposure.

\section{Perspectives}

Nanomedicine-based drug delivery systems can offer several beneficial features, for example they may help to increase the solubility of hydropobic drugs, improve pharmacokinetics attributes, reduce toxicity and increase therapeutic efficacy for small molecules drug. And they may increase stability, circulation kinetics, allow for cellular targeting and intracellular delivery for high molecular weight drugs. Nanomedicine-based drug delivery systems can be mainly categorized into synthetic systems and natural system based on their source of origin.

Synthetic systems have the advantage such as easy to scale-up, and their product quality can be easy be controlled and tailored. In chapter 2 of this thesis, we found that a novel nanogel and its PEG-coated formulation can effectively encapsulate mRNA and transfect cells. Potential improvements include the use of sheddable PEG-coating to restore an interactive activity after tissue disposition. Additionally, targeting ligands can be employed to improve cellular specificity and internalization of cells at the site of disease. The localized activity that was observed for the nanogels is important for example for mRNAs that can stimulate the immune system. For such therapeutic strategies localized activity that does not leak into peripheral tissues is important.

Although the Cre-recombinase reporter system is attractive as it is ultrasensitive and may be responsive to functional delivery of a single Cre-mRNA, this sensitivity is also a disadvantage. For many applications robust protein expression is required and this may not easily be deduced from the reporter experiments. This would be important to determine for future studies.

EVs from bacteria sources have the potential to become vaccine candidates as bacteria are easy to culture, and pathogen-associated molecular patterns are displayed on their surface. In chapter $\mathbf{5}$ of this thesis, we found that the EV yields from Grampositive bacteria E1162 are quite low which will hinder future clinical translation. This is probably due to the fact that EVs can hardly escape the relatively thick wall of bacteria. The production yield of EVs from Gram-positive bacteria E1162 was 
increased after yajC knockout, however, it may still not high enough when large-scale clinical application is considered. Therefore, exploring other methods to increase the yield of MVs from Gram-positive bacteria E1162 are needed. And generally speaking, the yield of EVs from Gram-positive bacteria is low compared with EVs from their counterpart Gram-negative bacteria. So approaches to increase the production of EVs from Gram-positive bacteria would be important for this EV source.

Although high IgG level were detected when healthy mice were immunized with E1162 MVs in chapter 5, future studies should be focused on whether the elevated IgG antibodies can protect mice from E1162 infections still to be studied. In future experiments, immunized mice should be challenged with E1162 bacteria to see whether the MVs vaccines indeed can prevent mice from infection.

Besides methods to increase the yield of EVs, approaches which can increase the targeting and immunogenicity of EVs are also important. In this way, EVs can be delivered to the site of disease with improved specificity. In addition, increasing immunogenicity would result in better therapeutic efficacy. We showed that postinsertion methods work for both mammalian and bacteria EVs in chapter 6. However, information on the uptake, stability and biological function of those EVs in vivo is currently not available and consequently, future research providing this information is important for bringing this strategy forward.

\section{References}

Choi, Y. H. and H.-K. Han (2018). "Nanomedicines: current status and future perspectives in aspect of drug delivery and pharmacokinetics." Journal of Pharmaceutical Investigation 48(1): 43-60.

Farokhzad, O. C. and R. Langer (2009). "Impact of nanotechnology on drug delivery." ACS nano 3(1): 16-20.

Wahlich, J., et al. (2019). Nanomedicines for the Delivery of Biologics, Multidisciplinary Digital Publishing Institute. 


\section{Appendices}

Samenvatting

Curriculum vitae

List of publications

Acknowledgements 


\section{Samenvatting}

Nanogeneeskunde is de toepassing van nanotechnologie bij het diagnosticeren en behandelen van ziekten. Deze nanogeneesmiddelen kunnen worden verkregen via chemische synthese of uit natuurlijke bronnen. Ontwikkelingen in de synthetische nanogeneeskunde hebben verschillende afgiftesystemen voor medicijnen opgeleverd zoals micellen, nanogelen, dendrimeren en liposomen. Van nature voorkomende en endogene nanosystemen omvatten bijvoorbeeld extracellulaire blaasjes die worden uitgescheiden door levende cellen. Zowel synthetische als natuurlijke nanogeneeskundesystemen tonen veel potentie als dragers van medicijnen, aangezien ze 1) gerichte afgifte van therapeutische middelen op de plaats van de ziekte mogelijk maken; 2) de oplosbaarheid van hydrofobe geneesmiddelen kunnen verbeteren; 3 ) gelijktijdige afgifte van twee of meer geneesmiddelmoleculen kunnen realiseren, waardoor combinatietherapie mogelijk is; 4) en de halfwaardetijd van medicijnmoleculen in de bloedsomloop kunnen verlengen door deze te beschermen tegen afbraak en snelle renale eliminatie.

In de afgelopen decennia is met gezamenlijke inspanningen van zowel de academische wereld als de industrie een aanzienlijk aantal op nanogeneeskunde gebaseerde producten beschikbaar gekomen voor klinische toepassingen. Bovendien is er de laatste jaren een toenemende belangstelling voor geneesmiddelen op basis van nucleïnezuren. Bestaande systemen voor medicijnafgifte zijn echter voornamelijk geschikt voor het afleveren van klein-moleculaire geneesmiddelen, terwijl het afleveren van nieuw opkomende geneesmiddelen zoals sterk geladen nucleïnezuren met een hoog molecuulgewicht een uitdaging blijft.

In hoofdstuk 2 wordt de karakterisering van een op poly (amido amine) gebaseerde nanogel beschreven. Deze nanogels konden worden beladen met cre-mRNA. In vitro studies toonden aan dat niet-gecoate nanogelen superieur waren in het transfecteren van cellen in vergelijking met hun met poly (ethyleenglycol) gecoate tegenhangers. Bovendien werd transfectie van Cre-recombinase-mRNA getest in Ai9 Crerecombinase-reportermuizen na subcutane injectie. In tegenstelling tot de in vitro waarnemingen waren hier de poly (ethyleenglycol) gecoate nanogelen superieur in het aantal cellen dat werd getransfecteerd in het subcutane injectiegebied, gevolgd door de niet-gecoate nanogelen. De injectie van vrij mRNA vertoonde nagenoeg geen activiteit. Lekkage van mRNA-expressie naar andere weefsels was vrijwel afwezig, wat de sterk gelokaliseerde activiteit van nanogel-gemedieerde mRNA afgifte aantoont.

In hoofdstuk $\mathbf{3}$ bespreken we de mogelijkheid om een nieuw nanogeneeskundig platform in de vorm van extracellulaire blaasjes (EV's), te gebruiken als dragers 
voor nucleïnezuren. Omdat EV's biologische ladingen zoals DNA / RNA en eiwitten functioneel kunnen overbrengen naar ontvangende cellen, zullen ze zeer waarschijnlijk ook de overdracht van exogene therapeutische nucleïnezuren vergemakkelijken. Hun veelzijdige moleculaire compositie maken manipulatie van hun inhoud en eigenschappen mogelijk. Kortom, EV's zijn een efficiënt en veilig alternatief voor het toedienen van nucleïnezuren.

Naast de potentie van EV's als medicijndrager hebben bacteriële EV's ook de mogelijkheid om als vaccinkandidaten te dienen tegen bacteriële infecties. Ten eerste zijn pathogeen-geassocieerde moleculen op het oppervlak van deze bacteriële EV's in staat om zowel aangeboren als adaptieve immuunresponsen te ontketenen. Ten tweede zijn de van bacteriën afgeleide EV's niet-repliceerbaar, wat als veilig wordt beschouwd. In hoofdstuk $\mathbf{4}$ hebben we voornamelijk de isolatieprocedures en karakterisatiemethoden voor bacteriële EV's besproken. Daarnaast komen ook technieken aan bod om nauwkeurige modificatie van membraanblaasjes (MV's) mogelijk te maken die helpen om veilige, zeer immunogene en efficiënte vaccins te verkrijgen. Het succesvolle ontwikkelingsverhaal van Bexsero ${ }^{\circledR}$, een commercieel verkrijgbaar EV-gebaseerd vaccinproduct tegen infectie met Neisseria meningococcus, wordt ook besproken.

Aangezien EV's van grampositieve bacteriën minder goed bestudeerd zijn dan hun tegenhangers van gramnegatieve bacteriën, blijft de manier waarop EV's ontsnappen uit de dikke celwanden van grampositieve bacteriën een onopgelost mysterie. Kennis over de immunogeniteit van deze EV's ontbreekt eveneens. Daarom worden in hoofdstuk 5 de grampositieve bacteriën Enterococcus faecium E1162 en zijn isogene mutanten onderzocht. We bestudeerden de morfologie, grootte, opbrengst, eiwitsamenstellingen en immunogeniciteit van MV's die vrijkomen uit de nosocomiale pathogeen grampositieve bacterie E. faecium wild-type (WT) stam E1162 en twee E1162 isogene mutanten $\triangle$ yajC en $\triangle$ AltA. Verschillende MVssubpopulaties werden waargenomen onder de MV's van E1162 en zijn mutanten. Kwalitatieve en kwantitatieve proteomics van MV's onthulden dat er een selectief eiwitsorteringsmechanisme is tijdens MV-biogenese. Bovendien toonde analyse aan dat zowel E1162 WT MV als E1162 $\triangle$ yajC MV een grotere hoeveelheid potentieel immunogene eiwitten bevatten zoals Pbp5, LysM, AdcAfm 2410 en AdcAfm 2344 in vergelijking met E1162 $\Delta$ AltA. Ten slotte werd aangetoond dat zowel E1162 als $\triangle y a j C M V ' s$ cytokine-afgifte en een langdurige immuunrespons in een muizenmodel initieerden, wat suggereert dat E. faecium-afgeleide MV's veelbelovende vaccinkandidaten zijn tegen $\mathrm{E}$. faecium-infectie.

Om de doelgerichtheid en immunogene eigenschappen van bacteriële EVs te verbeteren, werd een eenvoudige post-insertie benadering onderzocht in 
hoofdstuk 6. DSPE-PEG-biotine lipiden kunnen worden opgenomen in de fosfolipide bilaag membranen van EVs van zowel zoogdiercellen als bacteriën. De introductie van biotine in het EV-membraan opent de mogelijkheid om de EVs te decoreren met streptavidine gekoppelde antigeenconjugaten. We ontdekten ook verschillen in het modificatieproces van zoogdier EV's ten opzichte van bacteriële EV's. In zoogdiersystemen assembleren de DSPE-PEG-biotine lipiden zich eerst in het donorcelmembraan, met als resultaat dat de EV's afkomstig van die cellen de lipiden integreren. Bij de bacteriën was geen insertie van DSPE-PEG-biotine lipiden detecteerbaar op het oppervlak van de cellen, maar een hoog biotine signaal kan worden gedetecteerd op het oppervlak van bacteriële EV's wat post-insertie van de lipiden suggereert.

Ten slotte werd in hoofdstuk 7 een co-cultuur systeem opgezet om de immunogeniciteit van outer membrane vesicles (OMVs) te onderzoeken. Dit heeft als doel een in-vitro platform te ontwikkelen voor de evaluatie van orale vaccinatiemogelijkheden van OMVs. De resultaten geven aan dat OMV's in staat zijn immuunresponsen van darmcellen/perifere bloed mononucleaire cels (PBMCs) in een gezamenlijk kweeksysteem op te wekken. Dit kan erop wijzen dat dit systeem kan worden gebruikt om de immuunstimulerende eigenschappen van gemanipuleerde OMV's voor vaccindoeleinden te onderzoeken. Het kan ook een aantrekkelijke opzet zijn om het effect van microbioom-afgeleide OMV's op darmcellen en hun communicatie met de systemische circulatie te testen. Verfijningen van de huidige opzet zouden een cytokine-expressieprofiel op lange termijn kunnen bestuderen en de effecten van indirecte blootstelling kunnen onderzoeken.

\section{Perspectieven}

Nanogeneeskundige systemen kunnen verschillende gunstige eigenschappen bieden voor medicijnafgifte. Ze kunnen bijvoorbeeld de oplosbaarheid van hydrofobe geneesmiddelen verhogen, de farmacokinetische eigenschappen verbeteren, de toxiciteit verminderen en de therapeutische werkzaamheid van medicijnen met kleine molecuulgewichten verhogen. Eveneens kunnen ze de stabiliteit en de circulatiekinetiek verhogen en de cellulaire targeting en intracellulaire afgifte voor geneesmiddelen met een hoog molecuulgewicht mogelijk maken. Op nanogeneeskunde gebaseerde systemen voor medicijnafgifte kunnen grofweg worden onderverdeeld in synthetische systemen en natuurlijke systemen op basis van hun oorsprong.

Hoewel het besproken Cre-recombinase reportersysteem aantrekkelijk is omdat het ultragevoelig is en enkel reageert op functionele afgifte van een Cre-mRNA, is 
deze gevoeligheid ook een nadeel. Voor veel toepassingen is robuuste inductie van eiwitexpressie vereist en dit kan niet gemakkelijk worden afgeleid uit de huidige reporter-experimenten. Dit zou belangrijk zijn om te bepalen voor toekomstige studies.

EV's uit bacteriële bronnen zijn potentiële vaccinkandidaten, aangezien bacteriën gemakkelijk te kweken zijn en pathogeen-geassocieerde moleculaire patronen op het EV oppervlak worden weergegeven. In hoofdstuk $\mathbf{5}$ van dit proefschrift hebben we ontdekt dat de EV-opbrengsten van grampositieve bacteriën E1162 vrij laag zijn, wat een klinische vertaling in de toekomst kan belemmeren. Dit komt waarschijnlijk doordat EV's nauwelijks kunnen ontsnappen aan de relatief dikke celwand van deze bacteriën. De productieopbrengst van EV's van grampositieve bacteriën E1162 was verhoogd na $\triangle y a j C$ knock-out, maar het is wellicht nog steeds niet hoog genoeg voor grootschalige klinische toepassing. Daarom zijn andere methoden nodig om de opbrengst van EV's van grampositieve E1162 bacteriën te verhogen. Over het algemeen is de opbrengst van EV's van grampositieve bacteriën laag in vergelijking met EV's van hun gramnegatieve tegenhangers. Dus onderzoek naar manieren om de productie van EV's van grampositieve bacteriën te verhogen, zouden belangrijk zijn voor deze EV-bron.

Hoewel in hoofdstuk 5 een hoog IgG-niveau werd gedetecteerd toen gezonde muizen werden geïmmuniseerd met E1162 MV's, zouden toekomstige studies zich moeten richten op de vraag of deze verhoging van IgG-antilichamen voldoende is om de muizen te beschermen tegen E1162-infecties. In toekomstige experimenten moeten geïmmuniseerde muizen worden uitgedaagd met E1162-bacteriën om te zien of de MVs-vaccins inderdaad infectie van muizen kunnen voorkomen.

Naast methoden om de opbrengst van EV's te verhogen, zijn ook benaderingen die de targeting en immunogeniciteit van EV's kunnen verhogen belangrijk. Op deze manier kunnen EV's met verbeterde specificiteit op de plaats van de ziekte worden afgeleverd. Bovendien zou een toenemende immunogeniciteit resulteren in een betere therapeutische werkzaamheid. We hebben aangetoond dat postinsertiemethoden werken voor zowel zoogdier- als bacteriële EV's in hoofdstuk 6. Toekomstig onderzoek kan zich richten op de opname, stabiliteit en biologische functie van deze EV's in vivo. 


\section{Curriculum vitae}

Linglei Jiang

Email address: jianglinglei@126.com

Mobile: +14436273390

\section{Education and academic training}

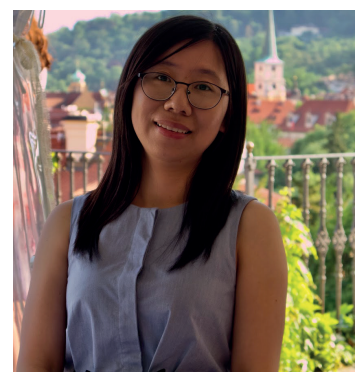

- Post-doc Training on Molecular \& Comparative Pathobiology

Johns Hopkins medicine, United States, 2020.3-current

- Ph.D. on Drug Innovation

Clinical Chemistry and Hematology

UMC Utrecht, the Netherlands, 2020.3

- M.S. Pharmaceutical Analysis

Pharmaceutical Analytical \& Solid-State Chemistry Research Center

Shanghai Institute of Materia Medica, University of Chinese Academy of Sciences, China, 2014.12

\section{Research activity}

2015.9 - 2020.3 Nanomedicine

Promotor: Prof. Raymond M. Schiffelers; co-promotor: Prof. Wim Hennink daily supervisor: Dr. Pieter Vader

Projects include:

- Enterococcus-derived membrane vesicles as a novel vaccine platform

European collaboration between department of clinical chemistry and haematology, department of medical microbiology UMC Utrecht (the Netherlands) and University of Tromsø (Norway).

- A lipid post-insertion method for functionalization the surface of mammalian-derived and bacteria-derived extracellular vesicles

Collaboration between department of clinical chemistry and haematology and department of medical microbiology, UMC Utrecht

- Intracellular delivery of nucleic acids (Cre-mRNA) by nanogel for gene therapy Collaboration between department of clinical chemistry and haematology and industry partner 20Med Therapeutics

- The effect of ultrasound on the release of extracellular vesicles

Collaboration between department of clinical chemistry and haematology and imaging division, UMC Utrecht

2020.3 - current

- Therapeutic use/biomarker discovery of extracellular vesicles

\section{Research interest}

- Therapeutic use of extracellular vesicles

- Drug delivery

- Pharmaceutical products development 


\section{List of publications}

\section{Publications related to this thesis}

Jiang, L., Vader, P., \& Schiffelers, R. M. (2017). Extracellular vesicles for nucleic acid delivery: progress and prospects for safe RNA-based gene therapy. Gene therapy, 24(3), 157.

Jiang, L., Schinkel, M., van Essen, M., \& Schiffelers, R. M. (2019). Bacterial membrane vesicles as promising vaccine candidates. European Journal of Pharmaceutics and Biopharmaceutics

Jiang, L., Luirink, J., Kooijmans, S. A., van Kessel, K. P., Jong, W., van Essen, M., ... \& Hennink, W. E. (2020). A post-insertion strategy for surface functionalization of bacterial and mammalian cell-derived extracellular vesicles. Biochimica et Biophysica Acta (BBA)-General Subjects, 129763.

Jiang, L., Paganelli F., Top J., Fens M., Mustafia N.,... ., \& Schiffelers (2021). Membrane Vesicles as nano-weapons for combating E. faecium infection (in preparation)

\section{Other publications}

Yuana, Y., Jiang, L., Lammertink, B. H., Vader, P., Deckers, R., Bos, C., ... \& Moonen, C. T. (2017). Microbubbles-Assisted Ultrasound Triggers the Release of Extracellular Vesicles. International journal of molecular sciences, 18(8), 1610.

llahibaks, N. F., Lei, Z., Mol, E. A., Deshantri, A. K., Jiang, L., Schiffelers, R. M., ... \& Sluijter, J. P. (2019). Biofabrication of Cell-Derived Nanovesicles: A Potential Alternative to Extracellular Vesicles for Regenerative Medicine. Cells, 8(12), 1509.

De Jong, O. G., Kooijmans, S. A., Murphy, D. E., Jiang, L., Evers, M. J., Sluijter, J. P., ... \& Schiffelers, R. M. (2019). Drug delivery with extracellular vesicles: from imagination to innovation. Accounts of chemical research, 52(7), 1761-1770.

Jiang, L.; Huang, Y.; Zhang, Q.; He, H.; Xu, Y.; Mei, X., Preparation and Solid-State Characterization of Dapsone Drug-Drug Co-Crystals. Cryst. Growth Des. 2014, 14, (9), 4562-4573.

He, H., Jiang, L., Zhang, Q., Huang, Y., Wang, J. R., \& Mei, X. (2015). Polymorphism observed in dapsone-flavone cocrystals that present pronounced differences in solubility and stability. CrystEngComm, 17(34), 6566-6574.

Xu, Y.; Jiang, L.; Mei, X., Supramolecular Structures and Physicochemical Properties of Norfloxacin Salts. Acta Crystallographica Section B. 2014, 70, (4), 750-760.

Xu, Y.; Jiang, L.; Huang, Y.; Wang, J.-R.; Mei, X., Solid-State Characterization and Transformation of Various Creatine Phosphate Sodium Hydrates. J. Pharm. Sci. 2014, 103, (11), 3688-3695. 
Wang, X.; Zhang, Q.; Jiang, L.; Xu, Y.; Mei, X., Isostructurality in six celecoxib cocrystals introduced by solvent inclusion. CrystEngComm. 2014, 16, (48), 1095910968.

Zhang, Q., Jiang, L., \& Mei, X. (2016). Thermodynamic and kinetic investigation of agomelatine polymorph transformation. Pharmaceutical development and technology, 21(2), 196-203.

\section{Patents}

One kind of a steviol glycoside and crystal preparation and use CN 103739639A inventors: Liping Zhu; Xuefeng Mei; Linglei Jiang; Bingqing Zhu

One kind of a steviol glycoside and crystal preparation and use CN103739640B inventors: Liping Zhu; Xuefeng Mei; Linglei Jiang; Bingqing Zhu

\section{Conference abstract}

Jiang, L., Vader, P., Hennink, W., \& Schiffelers, R. M. (2018). Remote loading of esterbased prodrugs and fluorescent labels using intravesicular hydrolases. Journal of Extracellular Vesicles, 7, 214-214. 


\section{Acknowledgements}

When reflecting my 4.5 years $\mathrm{PhD}$ life in the Netherlands, I can not feel more grateful to this wonderful journey ever. During this period of time, I conducted my research in a picturesque Dutch city Utrecht, a place with fairy tale scenery, and a place brought me a lot of serenity, inspiration, freedom and joy. I was very delighted to meet and work with a lot of excellent scientists and friends here, thanks a lot for sharing your valuable knowledge and interesting personal stories with me.

First of all, I would like to express my gratitude to my promotor Prof. Dr. Raymond Schiffelers. Ray, thanks a lot for providing this wonderful working opportunity in your lab which is so much in the frontier of the nanomedicine field. I like the interdisciplinary research lines in your lab, the projects I participated/heard are so interesting and fascinating. Thanks a lot for sharing your knowledge in science, setting up good collaboration for my projects, help me improve my writing and presenting skills. Besides, it was a lot of fun to attend your professor promotion party, the BBQ your family organized as well as our lab social get-together in Café Tilt. You are always so kind and encouraging! Hartelijk bedankt!

I would also like to extend my gratitude to Prof. Dr. Wim Hennink. Dear Wim, thanks a lot to your academic impact that I got to know about Utrecht University. Thanks a lot for setting up the $\mathrm{PhD}$ enrollment interview for me, helping me integrated into your David de wied gebouw Biopharmacy family, caring about my project progress, taking your time to have meeting with me and correcting my thesis. Your dedication and passion towards work sets a good example for me as a scientist and I really respect you.

Sincere thanks goes to my daily supervisor Dr. Pieter Vader, really thanks for teaching me a lot of lab techniques during the early stage of my $\mathrm{PhD}$ and got me involved in lab, and thanks for always providing insights to my projects and help me revise my manuscripts and thesis efficiently. Also many thanks for sharing your funding writing experience, Wish you all the best with your career!

Thanks my main collaborators from department of microbiology. Dear Prof. Dr. Rob Willems, thank you so much for your help with my work in your department, I can not make progress with my enterococcus project without your kind help. You're always so patient to explain the question I have, send me the latest bacterial membrane vesicle-related paper and correcting my manuscript. It also fun to hear your holiday stories. Dear Dr. Janetta Top and Dr. Fernanda Paganelli, thanks a lot for sharing with me a lot of materials, reagents and protocols, as well as help me correct my manuscript. I am really admire your good working attitude and will learn from you. Dr. Kok van Kessel, thanks a lot for helping me when I need to use the 
flow cytometry for bacteria in your lab, and help me found a 12-year old antibody in house which turns out to be amazing. Very glad to learn the research experience from you.

Thanks Dr. Marcel Fens for your kind help with my in vivo study in this thesis, and also very kind of you to explain fun Dutch culture I observe.

Thank you Dr. Zhiyong Lei for your kind help with my in vivo study in UMC. I really appreciated that the abundant knowledge you have in biomedical science, your good collaboration spirit and your dedication towards work. Wish you all the best with the translational research you're conducting!

I would also like to thank Prof. Dr. Mona Johannessen from UiT (The Arctic University of Norway) for helping me with the proteomics study in this thesis, I cannot make it without your kind help. Thanks Mr. Jack-Ansgar Bruun and Mr. Toril for performing the experiment and analyzing the data. Thanks Dr. Fatemeh Askarian from Norwegian University of life sciences for setting up this collaboration with Dr. Mona during your visit to UMC Utrecht.

Thanks a lot to Dr. Joen Luirink from Vrie University van Amsterdam for providing your hypervisiculate bacteria strain for my study. And I am a big fan about the elegant work you and your colleagues has achieved. I got a lot inspiration from it. And thanks Dr. Wouter Jong from Abera for your kind help with my projects.

I would like to thank Prof. Dr Johan Hassen, Dr. Linette Willemsen and PhD candidate Veronica Ayechu Muruzabal for the help with setting-up the co-culture system in this thesis.

Many thanks to the master students (back then) I have collaborated with. Najrana, so glad to work with you for the enterococcus EV project and I also learn a lot from your science perspective and touched by your personal story. It was a fun time to work together, and I felt grateful that you were also helping me understand your culture. All the best with your good work and happy life in the Netherlands. Max, very glad to work together with you, you're such a talented and passionate scientist, thanks a lot for the hard work during your internship. Wish you all the best with your current PhD work and future career towards a successful neuron surgeon as well. Thanks for giving me the nice book 'The UnDutchables', I like it a lot and also recommend to other international colleagues/friends in the Netherlands. Michelle, thanks for your devotion and a good collaboration for our review, also wish you a fruitful PhD journey and a bright future!

Very special thanks also goes to my Pls in Johns Hopkins University, Dr. Kenneth Witwer and Dr. Vasiliki Machairaki. When I was half-way on my thesis, I've started my new job in your labs. Thanks a lot for providing this wonderful research 
opportunity for me as well as helped me set up my life when I just landed in United States so that I can continue writing my thesis. Your kind help means a lot to me in this difficult pandemic year.

Thanks Yui (Wariya) and Dan, very very honored to have you as my paranymphs. Dear Yui, It was such a wonderful memory to be PhD students together in the Netherland. We witness each other's growth at work and have fun together as 'partner in crime'. Very glad that we went to the Fort Blauwkapel for conference together, we organized Asian food adventure trip and participate the annual bake-off together in lab. I am also impressive by all the nice paper you wrote and get inspired by your travelling stories. Sincerely wish you a lot of success and happiness! Dear Dan, very appreciate your science attitudes and elegant work, and you're always willing to help people too, also really appreciate your passion towards cycling. Thank you for helping me improving my English and work too. Very fun to watch football match at Galgenwaard together and watch you roast a goose. Keep going good work and stay cool!

Thanks Anil for the happy time working together and fun in lab outing, I was always amazed at your happy dance, thanks for calling me in the pandemic! And Dear Virgy (Virginia) really miss the time that we encouraged each other to have fun both at work and at life, I heard so many fascinating stories about Italy from you, and I also wanna show you my hometown when you made your world tour in China! Wish you 'Felicita'! Aida, I still remember the time we took ADDT course in Leiden and the time we enjoyed caipirinha together. Time flies and wish you a lot of success with your current research and a lot of fun. Birgit, it was so fun to hear your work at Van Creveldkliniek and happy to read your paper in reseachgate too, and it is also fun to hear how you coach little kids for skiing in Austria. Thanks a lot for borrowing your skiing gear and teach me in the lab Winterburg skiing tour. I wish you keep up you good work and have fun! Hope to see you in future as well. Demian, beste bro, your nano-polymer research is so interesting, and I appreciate your hard work and good collaboration spirit. Thanks for always being willing to help me when I need to make telephone calls in Dutch, and very touched the time that you were helping my friend find the GP in a very urgent morning! Wish you be happy at work and at life like you always do. Marc, congrats on the funding you receive recently for your cofounded company, and I really admire your presenting skills and coordination skills. All the best! Tessa, all the best with your research on thrombosis and haemostasis in lab, Smile like you always do! Stephanie, you're always so creative with your research and lab borrel decoration, thanks for giving me the nice knitted Rooster you made in my Chinese new year, very touched! And thank you for being my dutch advisor too! Sara, was very glad to share the office with you, and thanks a lot for the encouragement and fun together. Zonne, very appreciate your calm and organized work style, all the best! Martijn(tje), you did such cool lipid nanoparticle work the 
time we met, and thanks a lot for filling beers in the lab, so we can have achoholic therapy when we need it, and also we can celebrate with it for the progress we made. You had many talents and a kind heart, wish you a lot of joy at work and at life. Dear Ominia, I really like your recent publication and I am looking forward to read more good work from you, keep up with your good work and joyful life in the Netherlands. Mariona, very glad that get to know you and sometimes still see you were working hard in the evening in UMC, your hard work will be paid off for sure, looking forward to your upcoming nice PhD work! Btw, I am also highly appreciate your Holloween costume. Pol, glad to meet you in UMC, and wish you all the happiness and success with your PhD. Khalid, also very glad to meet you in UMC, and all the best with your future career and life. Lina, very glad to know you when you were a master student in our lab in Utrecht University, and also very fun to hear your following PhD stories from Lyon. Thanks for telling me the stories about Moomin! And it was very good memory we cycled together to Hilversum from Utrecht, and we did the sailing in ljsselmeer too! All the best with your PhD work, and be happy! And thanks also goes to LKCH (now CDL) lunch meeting external friend Vania from department of immunology, wish you a lot of success and fun at work and life.

I also like to thank our colleagues Dr. Sander, Dr. Olivier, so glad to hear the good presentation and elegant research from you. And also fun to hear your 'talk show' right before Monday vesicle meeting. Nazma, Emma, Rowan, Marieke, Simon and Margarita, I learn a lot from your research experience and story too, bedankt and obrigada! Thanks Dr. Coen for always encouraging me to ask question on Friday morning meeting and Dr. Steven for giving critical thoughts about my work. Dear Dr. Yuana, very glad to get to know you during my $\mathrm{PhD}$ and thank you for sharing your knowledge, working experience and your culture.

Thanks Mr. Arnorld Koekman for your kind help with my work in lab, you can always find the instruments I need and instruct me, or sometimes help me fix it. Very appreciate this. And also very fun to attend the lab borrel (drinks) you and the other colleagues organized. Thanks Mr. Arjan Barendrecht for help with the fluorescent microscope and flow cytometer in lab, and also the lab borrel and photos you organized/took are awesome! Dear Silvie Sebastian, it was always so nice to talk with you in lab, you're so caring and kind. Thanks for the big smiles you drew on the reagents I ordered. And thanks for the warm messages when I moved to Baltimore. I like your nice sketch in lab and the wool hat your reagents bottles are wearing. Take care and hope we can meet again. Mr. Cor Seinen, thanks for the technical support for my EM and cryo-EM study. Mr. Marco Viveen, thanks for the help with the EM and share your knowledge too! Naomi, thanks for the happy time we have in lab, and fun to hear your holiday story and try your amazing cake too, all the best! Oh, you're obligated to travel to China in the future :P. Brigitte, Jerney, Annet, Jennifer, 
thanks for the good time at work together. Joukje, many thanks for helping with my visa and contract, Michel, thanks a lot for installing software and updating my working computer. Thanks Barbara for help arrange meeting with Wim and Mies, Joep for technical help in Biopharmacy lab.

Thanks to great PhD students I get to know from department of microbiology, Yuxi and Vincent, thanks for sharing your knowledge and what you have learn during the conference in your field with me, very fun to hear! Wish you all the success and joy for now and future. Dennis, it was very beneficial for me to discuss research with you, thank you for sharing your science insights. Paul, Elena glad and fun to meet you in lab too, all the best!

Thanks to all the great colleagues and friends in Department of Pharmaceutics! Dear Haili, it was really nice to have you in Utrecht, thank you for helping me integrated into work in lab and lab social activity. It was also a wonderful memory that we travelled to many European countries together Begium, France, Czech, Austria and Germany. And it is of my great honor to be your paranymph for your promotion. Toast to the good time that we witness each other's growth both at work and at life. I wish you a success career in Novatis and a happy life. Yanna, I really appreciate your passion about science and your happy personality, miss our good conversation when we met in UMC canteen. All the best with your new job and life. Dear Weiluan, Dandan, Yinan, Bo, Feilong, I am very grateful to meet you in the Netherlands, and I felt warmth when being together with you - our biopharmacy family, thanks for always being willing to help me at work and at life. Your successful career and fun life stories inspires me a lot, thanks for all the kindness! Boning, Jerry and Mengshan, wish you all the success with your research too. Yumao and Meng, very glad to have you as friends, thank you for being my 'Netherlandspedia', and always care and help me! I also wish you a lot of fun and success!

Many thanks goes to friends I met when I lived in Burgemeester ter pelkwijklaan. Jingwen, it would be a life-long memory for me when we cycled together to Fort bij Vechten. The beautiful scenery and sunshine reminds me scenes in Studio Ghibli's animation, and we also did a 'Fort escape' that day. And I also enjoy the time we went to stand-up paddling course, drifting along the beautiful canals in Bunnik, without your company and encouragement, I would never dare to try it. I also really appreciate your attitude towards good science, it inspires me a lot. And thanks for encouraging me to make progress in science too. Without your help, I could have not safely make it to States to continue my thesis writing and doing research too! Sincerely wish you all the best with your work in the Netherlands, in China, and the collaboration you have with United States. I also wish you and your family be very happy! Shuang, a lot of fun memories flashes when I was thinking of you. It was 
always fun to wave to each other when we were in the different end of the corridor in UMC. The best Croatia trip and the time we were in the great hall of Wiener Musikverein. And it was so fun to organize social together for our Burgemeester friends together. Keep your faith towards your good work and life and hope to see you again soon! Qiangbing, so nice that we were also working in the same lab. Really appreciate your hard work and passion about science. Keep going with your good gene delivery work. And many thanks for the help with my not-well-planned pandemic move! I wish you a lot of fun in lab, and also wish you a lot of success with your projects and future career. Keep in touch! Juntao, very glad to meet you in Burgemeester, and very fun to hear the funny experience you had as a fresh PhD student in the Netherlands. Appreciate your achievement you had made at work! And thanks a lot for help with my life in Burgemeester. All the best! Liling and Chao, thanks a lot for the great help in life and at work, I really value our friendship. Liling, still remember the time that we shared each other's story in UMC canteen and then we became friends. So glad we go to the CSC English course together and shopping together. you're my best cooking teacher! so happy to witness the growth of you and your babies too, hope you and Chao have a lot joy and success in the Netherlands. Xiaogang and Tao, Weiyang and Bohui, it was always delighted to chat with you on science/life over nice food and drinks. You're so sincere and fun, I wish you all the best for finishing up PhD work and then land a job you like. Xlaogang, I also want to say thank you for sharing your knowledge on EV field with me, it helps and inspires my work a lot. And happy to attend ISEV 2018 together in Spain. Many other very good PhD friends I met in Burgemeester includes Xiaoxu, Fenghua, Na, Xiao, Huiying, Yongbing, you inspired me a lot and I wish you all the best with your new/ upcoming position and role.

Thanks my piano teacher Henriëtte from Parnassos for bringing a lot beautiful musical stories and melodies to my PhD life and thanks Iliana from Agilecolor for the nice Indesign, PS, Al courses, and also helping out with the design of this thesis.

Many thanks to my supervisor during my master study, Prof. Dr. Xuefeng Mei, thanks for encouraging me during my PhD application time, give me suggestions on career selection, and thanks for sharing your experience both in academia and industry! And I also want to thank my friends in Shanghai, Xiuli, Jian, Qingqing and Dan Zhao, you always make me feel home when I flew back to Shanghai, and always so happy to hear your latest status at work and life. I really cherish this friendship and wish you all the best. Also thanks goes to my classmates Yun Xu and Liping, thank you for visiting me in the Netherlands and always caring about me. Qingshuo, thanks a lot for sharing your network and knowledge when I have questions. 
Sincere thanks also goes to Witwer and Vasso's lab members as well as friends I met in Baltimore Heather, Liuyi and Jingyi, thank you for all the help and fun during my final thesis writing phase! I also wish you a lot of success and happiness with your study/work in Hopkins.

Thanks my parents for giving me unconditional support and love during my PhD and always do. Listen to my happiness and sorrows, trying to understand different cultures together with me, be my best Michellin star chef, best tour guide when I was back home.

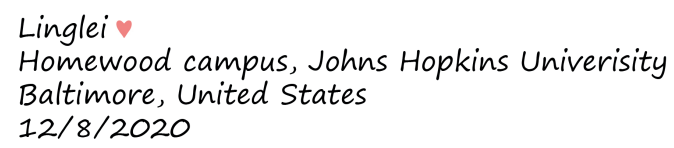


